\title{
AN ALMA SURVEY OF SUBMILLIMETER GALAXIES IN THE EXTENDED CHANDRA DEEP FIELD SOUTH: THE REDSHIFT DISTRIBUTION AND EVOLUTION OF SUBMILLIMETER GALAXIES
}

\author{
J. M. Simpson ${ }^{1}$, A. M. Swinbank ${ }^{1}$, Ian Smail ${ }^{1}$, D. M. Alexander ${ }^{1}$, W. N. Brandt ${ }^{2,3}$, F. Bertoldi ${ }^{4}$, C. De BreucK ${ }^{5}$, \\ S. C. Chapman $^{6}$, K. E. K. Coppin ${ }^{7}$, E. Da Cunha ${ }^{8}$, A. L. R. Danielson ${ }^{1}$, H. Dannerbauer ${ }^{9}$, T. R. Greve ${ }^{10}$, J. A. Hodge ${ }^{8}$, \\ R. J. Ivison ${ }^{11}$, A. Karim ${ }^{4}$, K. K. KnUdsen ${ }^{12}$, B. M. Poggianti ${ }^{13}$, E. Schinnerer ${ }^{8}$, A. P. ThOmson ${ }^{1}$, F. Walter ${ }^{8}$, \\ J. L. Wardlow ${ }^{14,15}$, A. Weiß ${ }^{16}$, AND P. P. VAN DER WERF ${ }^{17}$ \\ ${ }^{1}$ Institute for Computational Cosmology, Department of Physics, Durham University, South Road, Durham DH1 3LE, UK; j.m.simpson@dur.ac.uk \\ 2 Department of Astronomy \& Astrophysics, 525 Davey Lab, Pennsylvania State University, University Park, PA 16802, USA \\ ${ }^{3}$ Institute for Gravitation and the Cosmos, Pennsylvania State University, University Park, PA 16802, USA \\ ${ }^{4}$ Argelander-Institute for Astronomy, Bonn University, Auf dem Hügel 71, D-53121 Bonn, Germany \\ ${ }^{5}$ European Southern Observatory, Karl-Schwarzschild Straße, D-85748 Garching bei München, Germany \\ ${ }^{6}$ Department of Physics and Atmospheric Science, Dalhousie University, Halifax, NS B3H 3J5, Canada \\ ${ }^{7}$ Centre for Astrophysics Research, Science and Technology Research Institute, University of Hertfordshire, Hatfield AL10 9AB, UK \\ ${ }^{8}$ Max-Planck Institute for Astronomy, Königstuhl 17, D-69117 Heidelberg, Germany \\ ${ }^{9}$ Universität Wien, Institut für Astrophysik, Türkenschanzstraße 17, A-1180 Wien, Austria \\ ${ }^{10}$ Department of Physics and Astronomy, University College London, Gower Street, London WC1E 6BT, UK \\ ${ }^{11}$ Institute for Astronomy, University of Edinburgh, Blackford Hill, Edinburgh EH9 3HJ, UK \\ ${ }^{12}$ Department of Earth and Space Science, Onsala Space Observatory, Chalmers University of Technology, SE-43992 Onsala, Sweden \\ ${ }^{13}$ INAF-Astronomical Observatory of Padova, I-35122 Padova, Italy \\ ${ }^{14}$ Department of Physics \& Astronomy, University of California, Irvine, CA 92697, USA \\ ${ }^{15}$ Dark Cosmology Centre, Niels Bohr Institute, University of Copenhagen, Denmark \\ ${ }^{16}$ Max-Planck-Institut für Radioastronomie, Auf dem Hügel 69, D-53121 Bonn, Germany \\ ${ }^{17}$ Leiden Observatory, Leiden University, P.O. Box 9513, 2300 RA Leiden, The Netherlands \\ Received 2013 October 6; accepted 2014 April 16; published 2014 May 30
}

\begin{abstract}
We present the first photometric redshift distribution for a large sample of $870 \mu \mathrm{m}$ submillimeter galaxies (SMGs) with robust identifications based on observations with ALMA. In our analysis we consider 96 SMGs in the Extended Chandra Deep Field South, 77 of which have 4-19 band photometry. We model the SEDs for these 77 SMGs, deriving a median photometric redshift of $z_{\text {phot }}=2.3 \pm 0.1$. The remaining 19 SMGs have insufficient photometry to derive photometric redshifts, but a stacking analysis of Herschel observations confirms they are not spurious. Assuming that these SMGs have an absolute $H$-band magnitude distribution comparable to that of a complete sample of $z \sim 1-2$ SMGs, we demonstrate that they lie at slightly higher redshifts, raising the median redshift for SMGs to $z_{\text {phot }}=2.5 \pm 0.2$. Critically we show that the proportion of galaxies undergoing an SMG-like phase at $z \geqslant 3$ is at most $35 \% \pm 5 \%$ of the total population. We derive a median stellar mass of $M_{\star}=(8 \pm 1) \times 10^{10} M_{\odot}$, although there are systematic uncertainties of up to $5 \times$ for individual sources. Assuming that the star formation activity in SMGs has a timescale of $\sim 100 \mathrm{Myr}$, we show that their descendants at $z \sim 0$ would have a space density and $M_{H}$ distribution that are in good agreement with those of local ellipticals. In addition, the inferred mass-weighted ages of the local ellipticals broadly agree with the look-back times of the SMG events. Taken together, these results are consistent with a simple model that identifies SMGs as events that form most of the stars seen in the majority of luminous elliptical galaxies at the present day.
\end{abstract}

Key words: galaxies: evolution - galaxies: high-redshift - galaxies: starburst

Online-only material: color figures, machine-readable tables

\section{INTRODUCTION}

In the local universe $\sim 60 \%$ of the total stellar mass is in early-type and elliptical galaxies (Bell et al. 2003). These galaxies lie on a tight "red sequence" (Sandage \& Visvanathan 1978; Bower et al. 1992; Blanton et al. 2003), follow welldefined scaling relations (the fundamental plane), and show correlations between the age and velocity dispersion $(\sigma)$ of their stellar population. Typically, the most massive ellipticals have velocity dispersions of $\sigma \sim 200-400 \mathrm{~km} \mathrm{~s}^{-1}$, with estimated luminosity-weighted stellar ages of $\sim 10-13 \mathrm{Gyr}$ (Nelan et al. 2005). Recently, near-infrared (NIR) spectroscopy of quiescent, red galaxies at $z \sim 1.5-2$, the potential progenitors of elliptical galaxies (see van Dokkum et al. 2004), has suggested that the stellar populations in these galaxies have a typical age of 1-2 Gyr (e.g., Whitaker et al. 2013; Bedregal et al. 2013). Taken together, these results suggest that the bulk of the stellar mass in elliptical galaxies formed early in the history of the universe, at redshifts $z>2$. However, it has proved challenging to study the progenitors of these galaxies as the most massive, star-forming galaxies at $z>2$ are also the most dust obscured (Dole et al. 2004; LeFloc'h et al. 2009). One route to uncovering these dusty starbursts is to search at submillimeter (submm) wavelengths, where the shape of the spectral energy distribution (SED) of the far-infrared (FIR) dust emission means that cosmological fading is negated by the strongly increasing flux density of the SED. For sources at a fixed luminosity, this "negative $k$-correction" results in an almost constant apparent flux density in the submm over the redshift range $z=0.5-7$ (see the review by Blain et al. 2002).

The earliest surveys aimed at searching for distant submillimeter galaxies (SMGs), particularly with the SCUBA camera on the James Clerk Maxwell Telescope (JCMT), uncovered moderate numbers of submm sources with $850 \mu \mathrm{m}$ flux densities of $S_{v}=5-15$ mJy (e.g., Smail et al. 1997; Hughes et al. 1998; Barger et al. 1998; Eales et al. 1999; Coppin et al. 2006). 
However, the coarse beam size of single-dish submm telescopes $\left(\sim 15^{\prime \prime}\right.$ for the JCMT at $\left.850 \mu \mathrm{m}\right)$ meant that resolving these submm sources into their constituent SMGs (and so determining their basic properties, such as redshift and luminosity) was impossible without significant assumptions about the properties of their multi-wavelength counterparts. For example, the correlation between the FIR and radio flux density of starforming galaxies could be employed (e.g., Ivison et al. 1998, 2000), as deep $1.4 \mathrm{GHz}$ radio imaging with the Very Large Array (VLA) provides the sub-arcsecond resolution required to accurately locate the counterpart to the submm emission (Ivison et al. 2002, 2004, 2007; Bertoldi et al. 2007; Biggs et al. 2011; Lindner et al. 2011). However, in typical surveys, radio imaging only identifies $\sim 50 \%-60 \%$ of the SMGs brighter than $S_{850 \mu \mathrm{m}}>5 \mathrm{mJy}$ and furthermore is expected to miss the counterparts of the most distant SMGs due to the disadvantageous radio $k$-correction. Despite this low identification rate, this technique has facilitated extensive follow-up of the counterparts of SMGs, and spectroscopy has shown that the radio-identified subset of the population has a redshift distribution that peaks at $z \sim 2.3$ (Chapman et al. 2005). These observations confirmed that SMGs have luminosities comparable to local ultraluminous infrared galaxies (ULIRGs), but crucially demonstrated that the space density of ULIRGs at $z \sim 2$ is $\sim 1000 \times$ higher than at $z=0$. With implied star formation rates (SFRs) of 100-1000 $M_{\odot} \mathrm{yr}^{-1}$, SMGs brighter than $1 \mathrm{mJy}$ may contribute up to half of the co-moving SFR density at $z \sim 2$ (Hughes et al. 1998; Blain et al. 1999; Smail et al. 2002; Wardlow et al. 2011; Casey et al. 2013).

Extensive multi-wavelength follow-up of the radio-identified subset of the SMG population, particularly with the Plateau de Bure Interferometer, measured the kinematic and structural properties of high-redshift SMGs, suggesting that SMGs have morphologies and gas kinematics consistent with merging systems (e.g., Tacconi et al. 2008; Engel et al. 2010; Swinbank et al. 2010a; Alaghband-Zadeh et al. 2012; Menéndez-Delmestre et al. 2013). Moreover, their large molecular gas reservoirs (which constitute $\sim 50 \%$ of the dynamical mass in the central few kpc; Greve et al. 2005; Riechers et al. 2010; Carilli et al. 2010; Bothwell et al. 2013) and SFRs mean they have the potential to form a significant proportion of the stars in a $\sim 10^{11} M_{\odot}$ galaxy in only $10^{8} \mathrm{yr}$. Taken with their space densities $\left(\sim 10^{-5} \mathrm{Mpc}^{-3}\right.$; Chapman et al. 2005; Wardlow et al. 2011), large black hole masses $\left(\sim 10^{8} M_{\odot}\right.$; Alexander et al. 2005, 2008), and clustering (e.g., Hickox et al. 2012), it appears likely that, like local ULIRGs, the luminous starbursts in SMGs are frequently triggered by major mergers of gas-rich galaxies (e.g., Ivison et al. 2012).

Comparison with numerical simulations (e.g., Granato et al. 2004; Di Matteo et al. 2005; Hopkins et al. 2006) also suggests that the starburst SMG phase will be followed by a dustenshrouded active galactic nucleus (AGN) phase, which evolves through an optically bright QSO phase before evolving passively into an elliptical galaxy. Moreover, assuming that the timescales for the AGN and QSO phases are short and that SMGs do not undergo significant gas accretion at much lower redshift, it has been shown via simple dynamical arguments that the SMGs can evolve onto the scaling relations observed for local, early-type galaxies at $z=0$ (e.g., Nelan et al. 2005; Swinbank et al. 2006). It has thus been speculated that SMGs are the progenitors of local elliptical galaxies (Lilly et al. 1999; Genzel et al. 2003; Blain et al. 2004; Swinbank et al. 2006; Tacconi et al. 2008; Hainline et al. 2011; Hickox et al. 2012).
These $850 \mu \mathrm{m}$-selected samples remain the best-studied SMGs. However, by necessity, the samples from which most of the follow-up has so far concentrated have been biased to the radio-identified and UV-bright subset of the population where their counterparts and redshifts could be measured. In 2009 we undertook a $310 \mathrm{hr}$ survey of the $0.5 \times 0.5 \mathrm{deg}$ ECDFS at $870 \mu \mathrm{m}$, with the LABOCA camera on APEX. This "LESS" survey (Weiß et al. 2009) detected 126 submm sources with $870 \mu \mathrm{m}$ fluxes $S_{870}>4.4 \mathrm{mJy}$, but still relied on radio and mid-infrared imaging Biggs et al. (2011) to statistically identify probable counterparts to $\sim 60 \%$ of the sources, with the remaining $\sim 40 \%$ remaining unidentified (Wardlow et al. 2011).

To characterize the whole population of bright SMGs in an unbiased manner, we have subsequently undertaken an ALMA survey of these 126 LESS submm sources. The ALMA data resolve the submm emission into its constituent SMGs, directly pinpointing the source(s) responsible for the submm emission to within $<0$ '.3 (Hodge et al. 2013), removing the requirement for statistical radio/mid-IR associations. Crucially, one of the first results from our survey demonstrated that just $\sim 70 \%$ of the "robust" counterparts from Biggs et al. (2011) were correct and that the radio and $24 \mu \mathrm{m}$ identifications only provide $\sim 50 \%$ completeness (Hodge et al. 2013), highlighting the potential biases in previous surveys (see also Younger et al. 2009; Barger et al. 2012; Smolčić et al. 2012). These ALMA identifications allow us for the first time to make basic measurements, such as the redshift distribution, for a complete and unbiased sample of SMGs.

In this paper, we exploit the extensive optical and NIR imaging of the ECDFS to derive the photometric redshift distribution, stellar mass distribution, and evolution of the ALMA-LESS (ALESS) SMGs. The paper is structured as follows. In Section 2 we present the multi-wavelength data used in our analysis, followed by a description of our method for measuring aperture photometry for the ALESS SMGs and sources in the field. In Section 3 we discuss the technique of SED fitting to determine photometric redshifts for the ALESS SMGs. Finally, in Section 4 we discuss the derived properties of the ALESS SMGs, such as redshift and stellar mass, and their comparison to similar high-redshift studies, concluding with remarks on their comparison to low-redshift populations. We give our conclusions in Section 5. Throughout the paper, we adopt a cosmology with $\Omega_{\Lambda}=0.73, \Omega_{\mathrm{m}}=0.27$, and $H_{0}=$ $71 \mathrm{~km} \mathrm{~s}^{-1} \mathrm{Mpc}^{-1}$, and unless otherwise stated, error estimates are from a bootstrap analysis. All magnitudes quoted in this paper are given in the $\mathrm{AB}$ magnitude system.

\section{OBSERVATIONS AND ANALYSIS}

\subsection{Sample Selection}

In this study we undertake a multi-wavelength analysis of the ALMA-detected submm galaxies from the catalog presented by Hodge et al. (2013) (see also Karim et al. 2013). To briefly summarize the observations, we obtained $120 \mathrm{~s}$ integrations of 122 of the original 126 LESS submm sources, initially identified using the LABOCA camera on the APEX telescope (Weiß et al. 2009). These Cycle 0 observations used the compact configuration, yielding a median synthesized beam of $\sim 1^{\prime \prime} .6 \times 1$.'. 2 . The observing frequency was matched to the original LESS survey, $344 \mathrm{GHz}$ (Band 7), and we reach a typical rms across our velocity-integrated maps of $0.4 \mathrm{mJy}_{\text {beam }}{ }^{-1}$. The observations are a factor of $3 \times$ deeper than LESS, but crucially the angular resolution is increased from $\sim 19^{\prime \prime}$ to $\sim 1^{\prime \prime} .5$. 
Table 1

Summary of Photometry

\begin{tabular}{lccc}
\hline \hline Filter & $\begin{array}{c}\lambda \text { effective } \\
(\mu \mathrm{m})\end{array}$ & $\begin{array}{c}\text { Detection Limit } \\
(3 \sigma ; \text { AB mag })\end{array}$ & Reference \\
\hline MUSYC WFI $U$ & 0.35 & 26.2 & Taylor et al. (2009) \\
MUSYC WFI $U_{38}$ & 0.37 & 25.3 & Taylor et al. (2009) \\
VIMOS $U$ & 0.38 & 28.1 & Nonino et al. (2009) \\
MUSYC WFI $B$ & 0.46 & 26.5 & Taylor et al. (2009) \\
MUSYC WFI $V$ & 0.54 & 26.3 & Taylor et al. (2009) \\
MUSYC WFI $R$ & 0.66 & 25.5 & Taylor et al. (2009) \\
MUSYC WFI $I$ & 0.87 & 24.7 & Taylor et al. (2009) \\
MUSYC Mosaic-II $z$ & 0.91 & 24.3 & Taylor et al. (2009) \\
MUSYC ISPI $J$ & 1.25 & 23.2 & Taylor et al. (2009) \\
HAWK-I $J$ & 1.26 & 24.6 & S. Zibetti et al. (in preparation) \\
TENIS WIRCam $J$ & 1.26 & 24.9 & Hsieh et al. (2012) \\
MUSYC Sofi $H$ & 1.66 & 23.0 & Taylor et al. (2009) \\
MUSYC ISPI $K$ & 2.13 & 22.4 & Taylor et al. (2009) \\
HAWK-I $K_{s}$ & 2.15 & 24.0 & S. Zibetti et al. (in preparation) \\
TENIS WIRCam $K_{s}$ & 2.15 & 24.4 & Hsieh et al. (2012) \\
SIMPLE IRAC $3.6 \mu \mathrm{m}$ & 3.58 & 24.5 & Damen et al. (2011) \\
SIMPLE IRAC $4.5 \mu \mathrm{m}$ & 4.53 & 24.1 & Damen et al. (2011) \\
SIMPLE IRAC $5.8 \mu \mathrm{m}$ & 5.79 & 22.4 & Damen et al. (2011) \\
SIMPLE IRAC $8.0 \mu \mathrm{m}$ & 8.05 & 23.4 & Damen et al. (2011) \\
\hline
\end{tabular}

The primary beam of ALMA is $\sim 17^{\prime \prime}$, which encompasses the original LESS error circles of $\lesssim 5^{\prime \prime}$. For full details of the data reduction and source extraction we refer the reader to Hodge et al. (2013). From the observations Hodge et al. (2013) construct a MAIN source catalog consisting of all detected SMGs obeying the following criteria: primary-beam-corrected map $\mathrm{rms}<0.6 \mathrm{mJy}$ beam $^{-1}$, signal-to-noise ratio $(\mathrm{S} / \mathrm{N})>3.5$, beam axial ratio $<2.0$, and lying within the ALMA primary beam. The resulting catalog contains 99 SMGs, extracted from 88 ALMA maps, which form the basis of the sample used in this paper. The positional uncertainty on each SMG is $<0$ '!3. Karim et al. (2013) demonstrate that the MAIN catalog is expected to contain one spurious source and to have missed one SMG. We remove three SMGs from our sample that lie on the edge of the ECDFS and so only have photometric coverage in two IRAC bands. Our final sample thus consists of 96 SMGs with precise interferometrically identified positions.

A supplementary catalog is also provided comprising sources extracted from outside the ALMA primary beam, or in lower quality maps (i.e., primary-beam-corrected map rms > $0.6 \mathrm{mJy} \mathrm{beam}^{-1}$ or axial ratio $>2.0$; see Hodge et al. 2013). In contrast to the MAIN catalog Karim et al. (2013) demonstrate that up to $\sim 30 \%$ of the supplementary sources are likely to be spurious, and as such we do not consider them in the main body of this work. However, we present the photometry of these supplementary sources with detections in more than three wavebands (14 out of 31 sources) in Appendix C, along with their photometric redshifts and derived properties.

\subsection{Optical and NIR Imaging}

The majority of our optical-NIR data come from the MUltiwavelength Survey by Yale-Chile (MUSYC; Gawiser et al. 2006), which provides $U$ - to $K$-band imaging (Taylor et al. 2009) of the entire $0.5 \times 0.5 \mathrm{deg}$ ECDFS region (detection limits are given in Table 1). We supplement this with $U$-band data from the GOODS/VIMOS imaging survey (Nonino et al. 2009), covering $\sim 0.17 \mathrm{deg}^{2}$ of the ECDFS. Although the additional $U$-band imaging only covers $\sim 60 \%$ of the ALESS SMGs, it is $\sim 2$ mag deeper than the MUSYC $U$-band imaging and provides a valuable constraint on SMGs undetected in the shallower imaging.

In addition, we include deep NIR $J$ and $K_{S}$ imaging from both the ESO-VLT/HAWK-I survey by S. Zibetti et al. (in preparation) and the Taiwan ECDFS NIR Survey (TENIS; Hsieh et al. 2012), taken using CFHT/WIRCAM. Both surveys are $\sim 1.5-2.0$ mag deeper than the MUSYC $J$ or $K_{S}$ imaging (Table 1). We include all three sets of $J$ and $K_{S}$ imaging in our analysis; however, where multiple observations exist we quote, or plot, a single value in order of the detection limit of the original imaging.

Finally, we include data taken as part of the Spitzer IRAC/MUSYC Public Legacy in ECDFS (SIMPLE; Damen et al. 2011) survey, which provides imaging at 3.6, 4.5, 5.8, and $8.0 \mu \mathrm{m}$ over the entire field. We note that the $5.8 \mu \mathrm{m}$ imaging is $\sim 2$ mag shallower than the other IRAC imaging.

To highlight the optical-NIR imaging, in Figure 1 we show $B I K_{S}$ and 3.6/4.5/8.0 $\mu \mathrm{m}$ false-color images for six example ALESS SMGs, spanning the full range of ALMA $870 \mu \mathrm{m}$ flux. Figure 1 demonstrates that the SMGs typically have counterparts in the NIR and where detected appear red in the $B I K_{S}$ color images. The full sample of 96 sources is shown in Appendix B in Figure 15.

\subsubsection{Photometry}

To derive photometric redshifts, we need to measure seeingand aperture-matched multi-band aperture photometry across all 19 filters available (see Table 1). First, we align all imaging to the ALMA astrometry. We use SExTRACTOR (Bertin \& Arnouts 1996) to create a source catalog for each image and match this catalog to the ALESS SMGs. The measured offsets in R.A. and decl. are $<0$ '. 3 in all cases and correspond to approximately a single pixel shift in the optical imaging and a sub-pixel shift in the NIR imaging.

After aligning all data to a common astrometric frame, we next seeing match the optical-NIR images. The resolution of the $U-K_{S}$ imaging is $\leqslant 1^{\prime \prime} .5$, and we convolve each image to the lowest resolution. We then measure photometry in a $3^{\prime \prime}$ diameter aperture using the IRAF package APPHOT. We initially center the aperture at the ALMA-identified position, but allow APPHOT to 

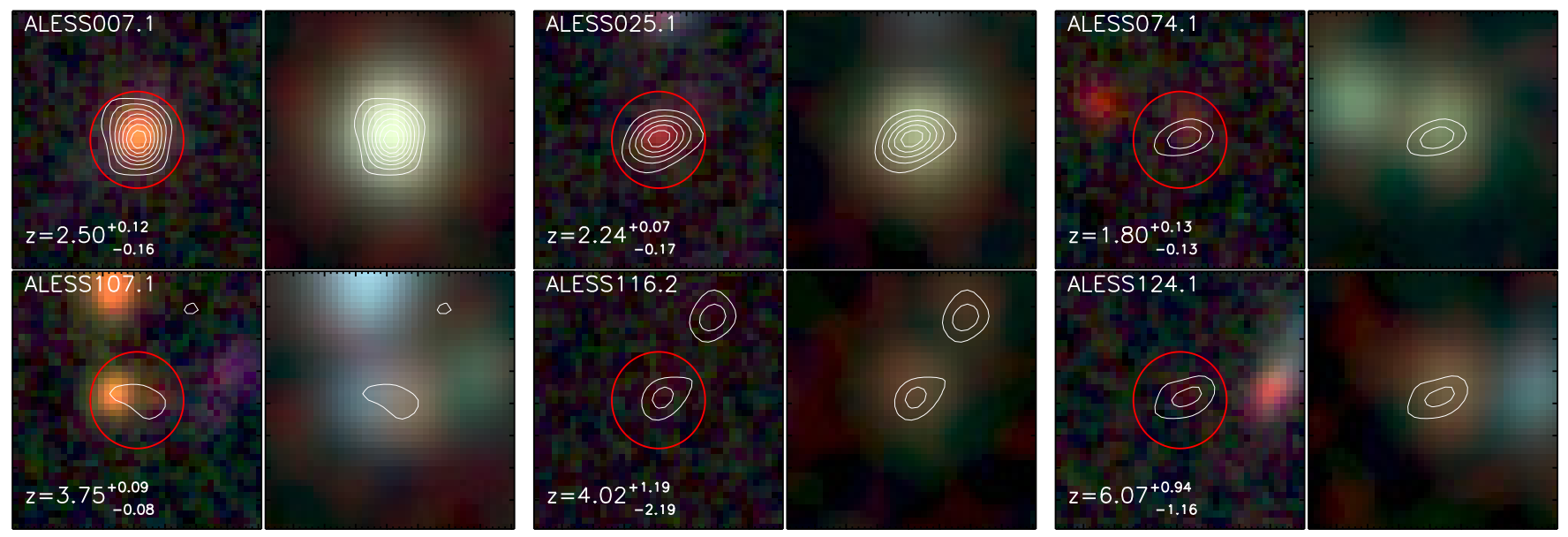

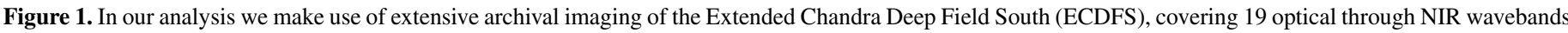

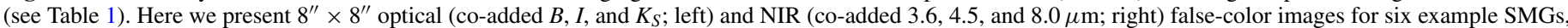

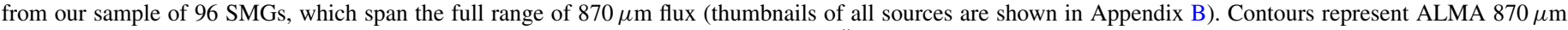

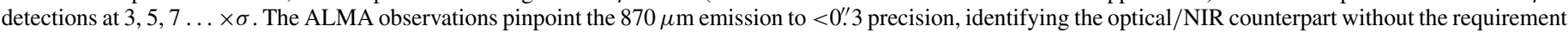

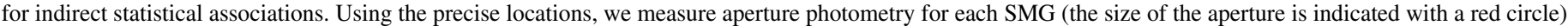
and derive photometric redshifts using the SED-fitting code HYPERZ; see Section 3.

(A color version of this figure is available in the online journal.)

re-center the aperture up to a shift of $<0$ '.5 from the original position. To correct for residual resolution differences in the $U-K_{S}$ imaging, we aperture correct our measurements to total magnitudes. We create a composite point-spread function (PSF), from 15 unsaturated point sources in each image, and derive the aperture correction as the ratio of the total flux in the composite PSF to the flux in the original $3^{\prime \prime}$ diameter aperture. The derived aperture corrections range from $f_{\text {tot }}(\lambda) / f_{\text {ap }}(\lambda)=1.18$ to 1.27. We assume that sky noise is the dominant source of uncertainty for these faint galaxies and estimate photometric errors by measuring the uncertainty in the flux in $3^{\prime \prime}$ apertures placed randomly on blank patches of sky in each image.

The resolution of the IRAC imaging is considerably poorer than the $U-K_{S}$ data, $2^{\prime \prime} 2$ at $8.0 \mu \mathrm{m}$. We therefore match the resolution of all the IRAC imaging to 2".2 FWHM and measure photometry in the same manner as the $U-K_{S}$, using a 3.'8-diameter aperture. To correct for the resolution difference between the IRAC and $U-K_{S}$ imaging, we again convert the IRAC photometry to total magnitudes. Following the same procedure as above, we measure the aperture correction from a composite PSF of 15 unsaturated point sources in each IRAC image. We measure aperture corrections of $f_{\text {tot }}(\lambda) / f_{\text {ap }}(\lambda)=1.49-1.89$, in the 3.6-8.0 $\mu \mathrm{m}$ wavebands, which are consistent with those estimated by the SWIRE team (Surace et al. 2005).

In all of the following analysis, we define detections if the flux is $3 \sigma$ above the background noise. The median number of filters covering each SMG is 14, and of the 96 SMGs in our sample, 77 are detected in $\geqslant 4$ wavebands. Of the remaining 19 sources, 10 are detected in 2 or 3 wavebands, and 9 are detected in $\leqslant 1$ waveband. We discuss these 19 sources in Section 3.2.3, where we show that a stacking analysis of IRAC and Herschel fluxes confirms that they correspond to FIR luminous sources, on average. We note that we do not perform any deblending of our photometry, and that we derive redshifts for 12 SMGs that are within $4^{\prime \prime}$ of a $3.6 \mu \mathrm{m}$ source of comparable, or greater, flux. In Table 2 we highlight sources that suffer significant blending and discuss the effects of blending in Section 3.2.1.

The photometry for the ALESS SMGs is given in Table 2, and in Figure 2 we show the $V, K_{S}$, and $3.6 \mu \mathrm{m}$ magnitude histograms. The ALESS SMGs have median magnitudes of $V=26.09 \pm 0.19, K_{s}=23.0 \pm 0.3$, and $m_{3.6}=21.80 \pm 0.17$ (58\%, 76\%, and $90 \%$ detection rates in each band). We note that at $3.6 \mu \mathrm{m}$ the Chapman et al. (2005) sample of radiodetected SMGs are a magnitude brighter than the ALESS SMGs $\left(m_{3.6}=20.63 \pm 0.18\right.$; Hainline et al. 2009)

\subsection{Herschel /SPIRE}

In this work we make use of observations at 250, 350, and $500 \mu \mathrm{m}$ using the Spectral and Photometric Imaging Receiver (SPIRE; Griffin et al. 2010) on board the Herschel Space Observatory (Pilbratt et al. 2010). The ECDFS was observed for $32.4 \mathrm{ks}$ at 250,350 , and $500 \mu \mathrm{m}$ in $\sim 1.8 \mathrm{ks}$ blocks as part of the Herschel Multi-tiered Extragalactic Survey (HerMES; Oliver et al. 2012). These data are described in Swinbank et al. (2014), the companion paper to this work studying the FIR properties of the ALESS SMGs. The final co-added maps reach a $1 \sigma$ noise level of $1.6,1.3$, and $1.9 \mathrm{mJy}$ at 250,350 , and $500 \mu \mathrm{m}$, respectively (see also Oliver et al. 2012), although source confusion means that the effective depth of these data is shallower than these noise levels imply. Deblended 250, 350, and $500 \mu \mathrm{m}$ fluxes for each ALESS SMG, along with the FIR properties, are presented in Swinbank et al. (2014).

$$
\text { 2.4. VLA/1.4 GHz }
$$

To study the radio properties of the ALESS SMGs, we utilize the VLA $1.4 \mathrm{GHz}$ imaging of the ECDFS. The observations come from Miller et al. (2008), and we use the catalog described in Biggs et al. (2011). These data reach an rms of $6.5 \mu \mathrm{Jy}$ in the central regions and a median rms of $8.3 \mu \mathrm{Jy}$ across the entire map. Biggs et al. (2011) extract a source catalog, complete to $3 \sigma$, and we obtain radio fluxes for the ALESS SMGs by cross-correlating the catalogs with a matching radius of $1^{\prime \prime}$. In our $1.4 \mathrm{GHz}$ stacking analysis we use the $1.4 \mathrm{GHz}$ map from Miller et al. (2013), a re-reduction of the original data achieving an improved typical map rms of $7.4 \mu \mathrm{Jy}$. We note that the $5 \sigma$ catalog from Miller et al. (2013) does not match any more SMGs than the catalog from Biggs et al. (2011), and that the $1.4 \mathrm{GHz}$ fluxes for individual sources all agree within their $1 \sigma$ errors. 
Table 2

\begin{tabular}{|c|c|c|c|c|c|c|c|c|c|c|c|c|c|c|c|}
\hline ID & MUSYC $U$ & MUSYC $U_{38}$ & VIMOS $U$ & $B$ & $V$ & $R$ & $I$ & $z$ & $J^{\mathrm{b}}$ & $H$ & $K^{b}$ & $3.6 \mu \mathrm{m}$ & $4.5 \mu \mathrm{m}$ & $5.8 \mu \mathrm{m}$ & $8.0 \mu \mathrm{m}$ \\
\hline$\overline{\text { ALESS } 1.1^{\mathrm{a}}}$ & $>26.18$ & $>25.29$ & $>28.14$ & $>26.53$ & $>26.32$ & $>25.53$ & $>24.68$ & $>24.29$ & $>24.88$ & $>23.06$ & $23.68 \pm 0.19^{*}$ & $22.81 \pm 0.06$ & $22.72 \pm 0.07$ & $22.28 \pm 0.19$ & $21.87 \pm 0.06$ \\
\hline ALESS 1.2 & $>26.18$ & $>25.29$ & $>28.14$ & $>26.53$ & $>26.32$ & $>25.53$ & $>24.68$ & $>24.29$ & $>24.88$ & $>23.06$ & $24.27 \pm 0.25$ & $22.81 \pm 0.06$ & $22.84 \pm 0.08$ & $21.99 \pm 0.15$ & $22.11 \pm 0.07$ \\
\hline ALESS $1.3^{\mathrm{a}}$ & $>26.18$ & $>25.29$ & $27.52 \pm 0.16$ & $>26.53$ & $>26.32$ & $>25.53$ & $>24.68$ & $>24.29$ & $>24.88$ & $>23.06$ & $23.98 \pm 0.20$ & $23.05 \pm 0.07$ & $22.92 \pm 0.09$ & $>22.44$ & $22.19 \pm 0.08$ \\
\hline ALESS $2.1^{\mathrm{a}}$ & $>26.18$ & $>25.29$ & $28.05 \pm 0.25$ & $>26.53$ & $26.30 \pm 0.26$ & $>25.53$ & $>24.68$ & $>24.29$ & $24.45 \pm 0.18$ & $\ldots$ & $23.03 \pm 0.09$ & $21.92 \pm 0.03$ & $21.66 \pm 0.03$ & $21.35 \pm 0.08$ & $21.83 \pm 0.06$ \\
\hline ALESS 2.2 & $>26.18$ & $>25.29$ & $>28.14$ & $>26.53$ & $>26.32$ & $>25.53$ & $>24.68$ & $>24.29$ & $>24.88$ & $\ldots$ & $>24.35$ & $23.32 \pm 0.09$ & $23.00 \pm 0.10$ & $>22.44$ & $22.12 \pm 0.07$ \\
\hline ALESS 3.1 & $>26.18$ & $>25.29$ & $\ldots$ & $>26.53$ & $>26.32$ & $>25.53$ & $>24.68$ & $>24.29$ & $>24.56^{*}$ & $>23.06$ & $23.24 \pm 0.10$ & $22.34 \pm 0.04$ & $21.71 \pm 0.03$ & $21.33 \pm 0.08$ & $21.03 \pm 0.03$ \\
\hline ALESS $5.1^{\mathrm{a}}$ & $24.83 \pm 0.08$ & $24.75 \pm 0.17$ & $\ldots$ & $23.15 \pm 0.01$ & $22.11 \pm 0.01$ & $21.33 \pm 0.01$ & $20.70 \pm 0.01$ & $20.60 \pm 0.01$ & $20.26 \pm 0.00$ & $\ldots$ & $19.79 \pm 0.00$ & $19.35 \pm 0.00$ & $19.50 \pm 0.00$ & $19.87 \pm 0.02$ & $20.09 \pm 0.01$ \\
\hline ALESS 6.1 & $24.58 \pm 0.07$ & $24.48 \pm 0.13$ & $24.52 \pm 0.01$ & $22.51 \pm 0.01$ & $21.70 \pm 0.00$ & $20.88 \pm 0.00$ & $19.90 \pm 0.00$ & $19.83 \pm 0.00$ & $19.73 \pm 0.00$ & $19.32 \pm 0.01$ & $19.81 \pm 0.00$ & $20.07 \pm 0.00$ & $20.36 \pm 0.01$ & $20.45 \pm 0.04$ & $20.81 \pm 0.02$ \\
\hline ALESS 7.1 & $25.97 \pm 0.22$ & $>25.29$ & $26.09 \pm 0.04$ & $24.63 \pm 0.05$ & $23.81 \pm 0.03$ & $23.06 \pm 0.03$ & $21.89 \pm 0.02$ & $21.81 \pm 0.03$ & $21.13 \pm 0.01$ & $20.68 \pm 0.03$ & $20.16 \pm 0.01$ & $19.65 \pm 0.00$ & $19.54 \pm 0.00$ & $19.47 \pm 0.02$ & $19.76 \pm 0.01$ \\
\hline ALESS 9.1 & $>26.18$ & $>25.29$ & $>28.14$ & $>26.53$ & $>26.32$ & $>25.53$ & $>24.68$ & $>24.29$ & $>24.88$ & $\ldots$ & $23.57 \pm 0.14$ & $21.76 \pm 0.02$ & $21.35 \pm 0.02$ & $21.03 \pm 0.06$ & $20.85 \pm 0.02$ \\
\hline ALESS 10.1 & $25.80 \pm 0.19$ & $\ldots$ & $25.58 \pm 0.03$ & $25.28 \pm 0.09$ & $25.35 \pm 0.17$ & $24.77 \pm 0.14$ & $24.36 \pm 0.20$ & $>24.29$ & $23.70 \pm 0.10$ & $\ldots$ & $22.74 \pm 0.07$ & $21.82 \pm 0.03$ & $21.39 \pm 0.03$ & $21.28 \pm 0.09$ & $21.06 \pm 0.04$ \\
\hline ALESS 11.1 & $>26.18$ & $>25.29$ & $28.06 \pm 0.25$ & $>26.53$ & $>26.32$ & $>25.53$ & $>24.68$ & $>24.29$ & $>24.88$ & $>23.06$ & $23.03 \pm 0.09$ & $21.78 \pm 0.02$ & $21.26 \pm 0.02$ & $20.89 \pm 0.06$ & $20.75 \pm 0.02$ \\
\hline ALESS 13.1 & $>26.18$ & $>25.29$ & $>28.14$ & $>26.53$ & $26.09 \pm 0.22$ & $25.35 \pm 0.23$ & $>24.68$ & $>24.29$ & $24.11 \pm 0.14$ & $>23.06$ & $22.54 \pm 0.06$ & $21.78 \pm 0.02$ & $21.51 \pm 0.02$ & $21.30 \pm 0.08$ & $21.14 \pm 0.03$ \\
\hline ALESS $14.1^{\mathrm{a}}$ & $>26.18$ & $>25.29$ & $\ldots$ & $>26.53$ & $>26.32$ & $>25.53$ & $>24.68$ & $>24.29$ & $24.00 \pm 0.13$ & $\ldots$ & $23.15 \pm 0.10$ & $22.14 \pm 0.03$ & $21.50 \pm 0.02$ & $21.16 \pm 0.07$ & $20.74 \pm 0.02$ \\
\hline ALESS 15.1 & $>26.18$ & $>25.29$ & $\ldots$ & $>26.53$ & $>26.32$ & $>25.53$ & $>24.68$ & $>24.29$ & $>23.22^{* *}$ & $\ldots$ & $>22.41^{* *}$ & $21.54 \pm 0.02$ & $20.93 \pm 0.01$ & $20.72 \pm 0.05$ & $20.64 \pm 0.02$ \\
\hline ALESS 15.3 & $>26.18$ & $>25.29$ & $\ldots$ & $>26.53$ & $>26.32$ & $>25.53$ & $>24.68$ & $>24.29$ & $>23.22^{* *}$ & $\ldots$ & $>22.41^{* *}$ & $22.76 \pm 0.06$ & $22.93 \pm 0.09$ & $>22.44$ & $>23.38$ \\
\hline ALESS 17.1 & $24.80 \pm 0.08$ & $25.02 \pm 0.21$ & $24.84 \pm 0.01$ & $24.20 \pm 0.03$ & $24.14 \pm 0.04$ & $23.72 \pm 0.06$ & $23.00 \pm 0.06$ & $22.83 \pm 0.08$ & $21.70 \pm 0.02$ & $21.14 \pm 0.05$ & $20.77 \pm 0.01$ & $19.98 \pm 0.00$ & $19.77 \pm 0.01$ & $19.96 \pm 0.02$ & $20.35 \pm 0.01$ \\
\hline ALESS 18.1 & $25.62 \pm 0.17$ & $>25.29$ & $25.54 \pm 0.03$ & $25.24 \pm 0.09$ & $25.06 \pm 0.09$ & $24.99 \pm 0.17$ & $24.31 \pm 0.20$ & $24.15 \pm 0.24$ & $22.64 \pm 0.04$ & $21.66 \pm 0.08$ & $21.13 \pm 0.02$ & $20.01 \pm 0.00$ & $19.68 \pm 0.00$ & $19.61 \pm 0.02$ & $20.28 \pm 0.01$ \\
\hline ALESS 19.1 & $>26.18$ & $>25.29$ & $26.81 \pm 0.09$ & $>26.53$ & $26.12 \pm 0.23$ & $>25.53$ & $>24.68$ & $>24.29$ & $>24.88$ & $\ldots$ & $23.60 \pm 0.14$ & $22.35 \pm 0.04$ & $21.85 \pm 0.03$ & $21.43 \pm 0.09$ & $21.58 \pm 0.04$ \\
\hline ALESS 19.2 & $26.04 \pm 0.24$ & $>25.29$ & $25.81 \pm 0.03$ & $25.05 \pm 0.07$ & $24.71 \pm 0.07$ & $24.48 \pm 0.11$ & $23.97 \pm 0.15$ & $24.02 \pm 0.21$ & $23.15 \pm 0.06$ & $\ldots$ & $22.29 \pm 0.04$ & $21.62 \pm 0.02$ & $21.68 \pm 0.03$ & $21.46 \pm 0.09$ & $21.60 \pm 0.05$ \\
\hline ALESS 22.1 & $>26.18$ & $>25.29$ & $\ldots$ & $>26.53$ & $25.96 \pm 0.20$ & $25.29 \pm 0.22$ & $>24.68$ & $>24.29$ & $\ldots$ & $\ldots$ & $\ldots$ & $20.11 \pm 0.00$ & $19.79 \pm 0.01$ & $19.64 \pm 0.02$ & $20.15 \pm 0.01$ \\
\hline ALESS 23.1 & $\ldots$ & $\ldots$ & $\ldots$ & & $\ldots$ & $\ldots$ & & & $>24.20$ & $\ldots$ & $>23.74$ & $23.11 \pm 0.08$ & $22.55 \pm 0.06$ & $21.78 \pm 0.12$ & $21.43 \pm 0.04$ \\
\hline ALESS 23.7 & $\ldots$ & $\ldots$ & $\ldots$ & & $\ldots$ & $\ldots$ & & & $\ldots$ & $\ldots$ & $\ldots$ & $22.90 \pm 0.06$ & $22.70 \pm 0.07$ & $>22.44$ & $>23.38$ \\
\hline ALESS 25.1 & $>26.18$ & $>25.29$ & $26.66 \pm 0.07$ & $25.74 \pm 0.14$ & $25.19 \pm 0.10$ & $24.73 \pm 0.14$ & $24.12 \pm 0.17$ & $23.95 \pm 0.20$ & $23.01 \pm 0.05$ & $\ldots$ & $21.52 \pm 0.02$ & $20.66 \pm 0.01$ & $20.35 \pm 0.01$ & $20.19 \pm 0.03$ & $20.44 \pm 0.02$ \\
\hline ALESS 29.1 & $>26.18$ & $>25.29$ & $\ldots$ & $>26.53$ & $>26.32$ & $>25.53$ & $>24.68$ & $>24.29$ & $>23.22^{* *}$ & $\ldots$ & $>22.41^{* *}$ & $21.85 \pm 0.02$ & $21.33 \pm 0.02$ & $20.86 \pm 0.05$ & $20.79 \pm 0.02$ \\
\hline ALESS 31.1 & $>26.18$ & $>25.29$ & $\ldots$ & $>26.53$ & $>26.32$ & $>25.53$ & $>24.68$ & $>24.29$ & $>24.88$ & $\cdots$ & $23.58 \pm 0.14$ & $22.30 \pm 0.04$ & $21.76 \pm 0.03$ & $21.27 \pm 0.08$ & $21.25 \pm 0.03$ \\
\hline ALESS 37.1 & $>26.18$ & $>25.29$ & $\ldots$ & $>26.53$ & $25.55 \pm 0.14$ & $24.29 \pm 0.09$ & $23.50 \pm 0.10$ & & $23.07 \pm 0.24^{* *}$ & $\ldots$ & $21.43 \pm 0.12^{* *}$ & $20.56 \pm 0.01$ & $20.30 \pm 0.01$ & $20.46 \pm 0.04$ & $20.60 \pm 0.02$ \\
\hline ALESS 37.2 & $>26.18$ & $>25.29$ & $\ldots$ & $>26.53$ & $26.10 \pm 0.22$ & $24.62 \pm 0.12$ & $\ldots$ & $\cdots$ & $>23.22^{* *}$ & $>23.06$ & $>22.41^{* *}$ & $22.33 \pm 0.04$ & $22.13 \pm 0.04$ & $21.73 \pm 0.12$ & $21.72 \pm 0.05$ \\
\hline ALESS 39.1 & $25.08 \pm 0.10$ & $>25.29$ & $\ldots$ & $25.06 \pm 0.07$ & $24.65 \pm 0.06$ & $23.98 \pm 0.07$ & $23.75 \pm 0.12$ & $23.20 \pm 0.10$ & $>23.22^{* *}$ & $\ldots$ & $22.04 \pm 0.04$ & $21.24 \pm 0.01$ & $20.90 \pm 0.01$ & $20.66 \pm 0.05$ & $20.67 \pm 0.02$ \\
\hline ALESS 41.1 & & $\ldots$ & $\ldots$ & $\ldots$ & $\ldots$ & $\ldots$ & & & & $\cdots$ & & $20.12 \pm 0.01$ & $19.83 \pm 0.01$ & $19.55 \pm 0.02$ & $19.51 \pm 0.01$ \\
\hline ALESS 41.3 & & $\ldots$ & $\ldots$ & $\ldots$ & $\ldots$ & $\ldots$ & $\ldots$ & & $\ldots$ & $\ldots$ & $\ldots$ & $22.39 \pm 0.04$ & $22.95 \pm 0.09$ & $>22.44$ & $22.45 \pm 0.10$ \\
\hline ALESS 43.1 & $>26.18$ & $>25.29$ & $28.10 \pm 0.26$ & $>26.53$ & $>26.32$ & $>25.53$ & $>24.68$ & $>24.29$ & $24.10 \pm 0.14$ & $\ldots$ & $22.48 \pm 0.05$ & $21.10 \pm 0.01$ & $20.67 \pm 0.01$ & $20.69 \pm 0.05$ & $21.33 \pm 0.04$ \\
\hline ALESS 45.1 & $>26.18$ & $>25.29$ & $>28.14$ & $>26.53$ & $>26.32$ & $>25.53$ & $>24.68$ & $>24.29$ & $>24.88$ & $\ldots$ & $22.67 \pm 0.06$ & $21.24 \pm 0.01$ & $20.80 \pm 0.01$ & $20.55 \pm 0.04$ & $20.75 \pm 0.02$ \\
\hline ALESS 49.1 & $>26.18$ & $>25.29$ & $\ldots$ & $24.61 \pm 0.05$ & $24.35 \pm 0.05$ & $24.10 \pm 0.08$ & $23.80 \pm 0.13$ & $24.07 \pm 0.22$ & $23.32 \pm 0.07$ & $\ldots$ & $22.63 \pm 0.06$ & $21.77 \pm 0.02$ & $21.49 \pm 0.02$ & $21.26 \pm 0.08$ & $21.27 \pm 0.03$ \\
\hline ALESS 49.2 & $26.11 \pm 0.25$ & $>25.29$ & $\ldots$ & $25.25 \pm 0.09$ & $25.23 \pm 0.10$ & $24.53 \pm 0.11$ & $23.98 \pm 0.15$ & $24.25 \pm 0.26$ & $23.60 \pm 0.09$ & $\ldots$ & $21.94 \pm 0.03$ & $20.88 \pm 0.01$ & $20.59 \pm 0.01$ & $21.01 \pm 0.06$ & $21.32 \pm 0.03$ \\
\hline ALESS 51.1 & $>26.18$ & $>25.29$ & $25.63 \pm 0.03$ & $24.97 \pm 0.07$ & $24.63 \pm 0.06$ & $23.65 \pm 0.05$ & $22.29 \pm 0.03$ & $22.09 \pm 0.04$ & $21.15 \pm 0.01$ & $20.73 \pm 0.03$ & $20.38 \pm 0.01$ & $19.47 \pm 0.00$ & $19.61 \pm 0.00$ & $19.80 \pm 0.02$ & $20.17 \pm 0.01$ \\
\hline ALESS 55.1 & $24.66 \pm 0.07$ & $24.60 \pm 0.15$ & $24.49 \pm 0.01$ & $24.25 \pm 0.04$ & $24.20 \pm 0.04$ & $24.05 \pm 0.07$ & $23.74 \pm 0.12$ & & $23.26 \pm 0.07$ & $>23.06$ & $22.72 \pm 0.11$ & $22.25 \pm 0.04$ & $22.07 \pm 0.04$ & $21.82 \pm 0.13$ & $21.56 \pm 0.07$ \\
\hline ALESS 55.2 & $>26.18$ & $>25.29$ & $>28.14$ & $>26.53$ & $>26.32$ & $>25.53$ & $>24.68$ & $>24.29$ & $>24.88$ & $>23.06$ & $>24.35$ & $>24.45$ & $>24.09$ & $>22.44$ & $>23.38$ \\
\hline ALESS 55.5 & $25.31 \pm 0.13$ & $25.15 \pm 0.24$ & $25.21 \pm 0.02$ & $24.74 \pm 0.06$ & $24.77 \pm 0.07$ & $24.75 \pm 0.14$ & $>24.68$ & & $24.42 \pm 0.18$ & $>23.06$ & $23.64 \pm 0.15$ & $22.70 \pm 0.05$ & $22.50 \pm 0.06$ & $22.35 \pm 0.20$ & $22.04 \pm 0.07$ \\
\hline ALESS 57.1 & $>26.18$ & $>25.29$ & $26.38 \pm 0.06$ & $24.86 \pm 0.06$ & $25.10 \pm 0.09$ & $24.65 \pm 0.13$ & $>24.68$ & $>24.29$ & $23.99 \pm 0.12$ & $22.66 \pm 0.19$ & $22.55 \pm 0.06$ & $21.64 \pm 0.02$ & $21.26 \pm 0.02$ & $20.95 \pm 0.06$ & $20.15 \pm 0.01$ \\
\hline ALESS 59.2 & $>26.18$ & $>25.29$ & $26.90 \pm 0.09$ & $26.21 \pm 0.20$ & $26.29 \pm 0.26$ & $>25.53$ & $>24.68$ & $>24.29$ & $24.24 \pm 0.15$ & $\ldots$ & $23.51 \pm 0.13$ & $22.92 \pm 0.06$ & $22.54 \pm 0.06$ & $>22.44$ & $22.31 \pm 0.09$ \\
\hline ALESS $61.1^{\mathrm{a}}$ & $>26.18$ & $>25.29$ & $\ldots$ & $>26.53$ & $>26.32$ & $>25.53$ & $24.39 \pm 0.21$ & $23.94 \pm 0.20$ & $23.00 \pm 0.05$ & $\ldots$ & $22.56 \pm 0.06$ & $22.83 \pm 0.06$ & $22.45 \pm 0.06$ & $>22.44$ & $22.02 \pm 0.07$ \\
\hline ALESS 63.1 & $>26.18$ & $>25.29$ & $\ldots$ & $25.96 \pm 0.17$ & $25.52 \pm 0.14$ & $24.79 \pm 0.14$ & $23.64 \pm 0.11$ & $23.23 \pm 0.11$ & $22.14 \pm 0.02$ & $\ldots$ & $21.26 \pm 0.02$ & $20.51 \pm 0.01$ & $20.29 \pm 0.01$ & $20.38 \pm 0.04$ & $20.63 \pm 0.02$ \\
\hline ALESS 65.1 & $>26.18$ & $>25.29$ & $\ldots$ & $>26.53$ & $>26.32$ & $>25.53$ & $>24.68$ & $>24.29$ & $>24.88$ & $\ldots$ & $>24.35$ & $23.33 \pm 0.09$ & $23.77 \pm 0.19$ & $>22.44$ & $22.88 \pm 0.14$ \\
\hline ALESS 66.1 & $20.91 \pm 0.00$ & $20.85 \pm 0.01$ & & $21.27 \pm 0.00$ & $21.22 \pm 0.00$ & $20.82 \pm 0.00$ & $20.66 \pm 0.01$ & $20.08 \pm 0.01$ & $21.21 \pm 0.05^{* *}$ & $\ldots$ & $20.28 \pm 0.04^{* *}$ & $19.41 \pm 0.00$ & $19.26 \pm 0.00$ & $19.08 \pm 0.01$ & $19.10 \pm 0.00$ \\
\hline ALESS 67.1 & $25.69 \pm 0.18$ & $>25.29$ & $25.24 \pm 0.02$ & $24.65 \pm 0.05$ & $24.30 \pm 0.05$ & $24.17 \pm 0.08$ & $23.52 \pm 0.10$ & $23.50 \pm 0.14$ & $22.41 \pm 0.03$ & $\cdots$ & $21.09 \pm 0.01$ & $20.26 \pm 0.01$ & $19.93 \pm 0.01$ & $19.80 \pm 0.02$ & $20.36 \pm 0.01$ \\
\hline ALESS 67.2 & $>26.18$ & $>25.29$ & $26.09 \pm 0.04$ & $25.57 \pm 0.12$ & $25.43 \pm 0.13$ & $25.10 \pm 0.19$ & $24.59 \pm 0.25$ & $24.12 \pm 0.23$ & $24.09 \pm 0.14$ & $\ldots$ & $22.98 \pm 0.08$ & $21.36 \pm 0.02$ & $21.13 \pm 0.02$ & $20.82 \pm 0.05$ & $21.48 \pm 0.04$ \\
\hline ALESS 68.1 & $>26.18$ & $>25.29$ & $>28.14$ & $>26.53$ & $>26.32$ & $>25.53$ & $>24.68$ & $>24.29$ & $>24.88$ & $\cdots$ & $>24.35$ & $23.34 \pm 0.09$ & $22.77 \pm 0.08$ & $>22.44$ & $22.09 \pm 0.07$ \\
\hline ALESS 69.1 & $>26.18$ & $>25.29$ & $\ldots$ & $>26.53$ & $>26.32$ & $>25.53$ & $>24.68$ & $>24.29$ & $>24.88$ & $\ldots$ & $22.74 \pm 0.07$ & $21.49 \pm 0.02$ & $21.08 \pm 0.02$ & $20.72 \pm 0.05$ & $21.02 \pm 0.03$ \\
\hline ALESS 69.2 & $>26.18$ & $>25.29$ & $\ldots$ & $>26.53$ & $>26.32$ & $>25.53$ & $>24.68$ & $>24.29$ & $>24.88$ & $\ldots$ & $24.29 \pm 0.25$ & $>24.45$ & $>24.09$ & $>22.44$ & $>23.38$ \\
\hline ALESS 69.3 & $>26.18$ & $>25.29$ & $\ldots$ & $>26.53$ & $>26.32$ & $>25.53$ & $>24.68$ & $>24.29$ & $>24.88$ & $\ldots$ & $>24.35$ & $>24.45$ & $>24.09$ & $>22.44$ & $>23.38$ \\
\hline ALESS $70.1^{\mathrm{a}}$ & $24.86 \pm 0.09$ & $24.37 \pm 0.12$ & $4.49 \pm 0.01$ & $23.72 \pm 0.02$ & $23.61 \pm 0.02$ & $23.47 \pm 0.04$ & $23.33 \pm 0.08$ & $23.34 \pm 0.12$ & $22.27 \pm 0.03$ & $\ldots$ & $21.16 \pm 0.02$ & $20.25 \pm 0.01$ & $20.03 \pm 0.01$ & $19.87 \pm 0.02$ & $20.21 \pm 0.01$ \\
\hline ALESS 71.1 & $>26.18$ & $>25.29$ & $\ldots$ & $25.36 \pm 0.10$ & $25.23 \pm 0.10$ & $24.28 \pm 0.09$ & $23.10 \pm 0.07$ & $23.03 \pm 0.09$ & $21.81 \pm 0.08^{* *}$ & $\ldots$ & $20.73 \pm 0.06^{* *}$ & $18.76 \pm 0.00$ & $18.08 \pm 0.00$ & $17.66 \pm 0.00$ & $17.79 \pm 0.00$ \\
\hline
\end{tabular}




层

Table 2

\begin{tabular}{|c|c|c|c|c|c|c|c|c|c|c|c|c|c|c|c|}
\hline \\
\hline ID & MUSYC $U$ & MUSYC $U_{38}$ & VIMOS $U$ & $B$ & $V$ & $R$ & $I$ & $z$ & $J^{b}$ & $H$ & $K^{\mathrm{b}}$ & $3.6 \mu \mathrm{m}$ & $4.5 \mu \mathrm{m}$ & $5.8 \mu \mathrm{m}$ & $8.0 \mu \mathrm{m}$ \\
\hline ALESS 71.3 & $26.18 \pm 0.27$ & $>25.29$ & $\ldots$ & $25.03 \pm 0.07$ & $25.13 \pm 0.10$ & $25.10 \pm 0.19$ & $>24.68$ & $>24.29$ & $>23.22^{* *}$ & $\ldots$ & $>22.41^{* *}$ & $23.40 \pm 0.10$ & $23.34 \pm 0.13$ & $>22.44$ & $>23.38$ \\
\hline ALESS 72.1 & $>26.18$ & $>25.29$ & $>28.14$ & $>26.53$ & $>26.32$ & $>25.53$ & $>24.68$ & $>24.29$ & $>24.88$ & $>23.06$ & $>24.35$ & $22.80 \pm 0.06$ & $22.91 \pm 0.09$ & $>22.44$ & $22.93 \pm 0.15$ \\
\hline ALESS 73.1 & $>26.18$ & $>25.29$ & $>28.14$ & $>26.53$ & $>26.32$ & $25.73 \pm 0.13$ & $24.00 \pm 0.15$ & $>24.29$ & $24.04 \pm 0.13$ & $\ldots$ & $23.57 \pm 0.14$ & $22.53 \pm 0.05$ & $22.41 \pm 0.06$ & $21.92 \pm 0.14$ & $21.59 \pm 0.04$ \\
\hline ALESS $74.1^{\mathrm{a}}$ & $>26.18$ & $>25.29$ & $27.55 \pm 0.16$ & $>26.53$ & $>26.32$ & $>25.53$ & $>24.68$ & $>24.29$ & $23.51 \pm 0.08$ & $\ldots$ & $22.36 \pm 0.05$ & $21.17 \pm 0.01$ & $20.77 \pm 0.01$ & $20.61 \pm 0.04$ & $21.18 \pm 0.03$ \\
\hline ALESS 75.1 & $24.65 \pm 0.07$ & $24.44 \pm 0.13$ & $\ldots$ & $23.58 \pm 0.02$ & $23.32 \pm 0.02$ & $23.11 \pm 0.03$ & $23.05 \pm 0.07$ & $23.22 \pm 0.11$ & $22.38 \pm 0.03$ & $\ldots$ & $22.01 \pm 0.03$ & $20.71 \pm 0.01$ & $20.06 \pm 0.01$ & $19.39 \pm 0.01$ & $18.68 \pm 0.00$ \\
\hline ALESS 75.4 & $26.09 \pm 0.25$ & $>25.29$ & $\ldots$ & $25.41 \pm 0.10$ & $25.21 \pm 0.10$ & $24.94 \pm 0.16$ & $>24.68$ & $>24.29$ & $24.43 \pm 0.18$ & $\ldots$ & $>24.35$ & $24.01 \pm 0.17$ & $>24.09$ & & \\
\hline ALESS 76.1 & $>26.18$ & $>25.29$ & $\ldots$ & $>26.53$ & $>26.32$ & $>25.53$ & $>24.68$ & $>24.29$ & $>23.22^{* *}$ & $\ldots$ & $>22.41^{* *}$ & $23.49 \pm 0.11$ & $23.06 \pm 0.10$ & $>22.44$ & $22.68 \pm 0.12$ \\
\hline ALESS 79.1 & $>26.18$ & $>25.29$ & $>28.14$ & $>26.53$ & $>26.32$ & $>25.53$ & $>24.68$ & $>24.29$ & $>24.88$ & $\ldots$ & $23.03 \pm 0.09$ & $21.70 \pm 0.02$ & $21.21 \pm 0.02$ & $20.90 \pm 0.06$ & $21.11 \pm 0.03$ \\
\hline LESS 79.2 & $26.08 \pm 0.25$ & $>25.29$ & $25.65 \pm 0.03$ & $24.97 \pm 0.07$ & $24.64 \pm 0.06$ & $24.31 \pm 0.09$ & $23.39 \pm 0.09$ & $23.31 \pm 0.12$ & $22.05 \pm 0.02$ & $\ldots$ & $20.89 \pm 0.01$ & $19.95 \pm 0.00$ & $19.75 \pm 0.00$ & $19.91 \pm 0.02$ & $20.45 \pm 0.02$ \\
\hline ALESS 79.4 & $>26.18$ & $>25.29$ & $27.73 \pm 0.19$ & $>26.53$ & $>26.32$ & $>25.53$ & $>24.68$ & $>24.29$ & $>24.88$ & $\ldots$ & $>24.35$ & $>24.45$ & $>24.09$ & $>22.44$ & $>23.38$ \\
\hline ALESS 80.1 & $>26.18$ & $>25$ & $68 \pm 0.18$ & $>26.53$ & $26.30 \pm 0.26$ & $>25.53$ & $24.66 \pm 0.26$ & $>24.29$ & $23.88 \pm 0.11$ & $\ldots$ & $22.28 \pm 0.04$ & $21.45 \pm 0.02$ & $21.12 \pm 0.02$ & $20.81 \pm 0.05$ & $21.34 \pm 0.04$ \\
\hline ALESS 80.2 & $>26.18$ & $>25$ & $7.00 \pm 0.10$ & $26.31 \pm 0.22$ & $25.83 \pm 0.18$ & $>25.53$ & $>24.68$ & $>24$ & $23.90 \pm$ & $\ldots$ & $22.51 \pm 0.05$ & $21.39 \pm 0.02$ & $21.14 \pm 0.02$ & $21.25 \pm 0.08$ & $21.94 \pm 0.06$ \\
\hline ALESS 82.1 & $>26.18$ & $>25.29$ & $>28.14$ & $>26.53$ & $>26.32$ & $>25.53$ & $>24.68$ & $>24.29$ & $24.56 \pm 0.27^{*}$ & $\ldots$ & $23.48 \pm 0.13$ & $22.19 \pm 0.03$ & $21.79 \pm 0.03$ & $21.61 \pm$ & $21.71 \pm 0.05$ \\
\hline ALESS 83.4a & $\ldots$ & $\ldots$ & $\ldots$ & $\ldots$ & $\ldots$ & $\ldots$ & $\ldots$ & $\ldots$ & $\ldots$ & $\ldots$ & $\ldots$ & $20.79 \pm 0.01$ & $21.01 \pm 0.02$ & $21.35 \pm 0.09$ & $22.06 \pm 0.07$ \\
\hline ALESS 84.1 & $25.81 \pm 0.20$ & $25.27 \pm 0.26$ & $5.30 \pm 0.02$ & $24.71 \pm 0.05$ & $24.60 \pm 0.06$ & $24.40 \pm 0.10$ & $24.03 \pm 0.15$ & $\ldots$ & $23.24 \pm 0.06$ & $22.45 \pm 0.16$ & $21.95 \pm 0.03$ & 21.0 & $20.71 \pm 0.01$ & $20.50 \pm$ & $20.70 \pm 0.02$ \\
\hline ALESS 84.2 & $>26.18$ & $>25.29$ & $26.56 \pm 0.07$ & $25.83 \pm 0.15$ & $25.33 \pm 0.11$ & $25.08 \pm 0.18$ & $4.71 \pm 0.27$ & $24.28 \pm 0.26$ & $2.83 \pm 0.04$ & $22.48 \pm 0.16$ & $21.65 \pm 0.02$ & & $20.81 \pm 0.01$ & $20.77 \pm$ & $21.33 \pm 0.04$ \\
\hline ALESS 87.1 & $\ldots$ & $\ldots$ & $5.33 \pm 0.02$ & $\ldots$ & $\ldots$ & & $\ldots$ & $\ldots$ & & $\ldots$ & $\ldots$ & $20.92 \pm 0.01$ & $20.68 \pm 0.01$ & $20.62 \pm 0.04$ & $20.50 \pm 0.02$ \\
\hline ALESS 87.3 & $\ldots$ & $\ldots$ & $>28.14$ & $\ldots$ & $\ldots$ & $\ldots$ & $\ldots$ & $\ldots$ & $\ldots$ & $\ldots$ & & $>24.45$ & $>24.09$ & $>22.44$ & $>23.38$ \\
\hline ALESS 88.1 & $25.65 \pm 0.17$ & $>25.29$ & $25.51 \pm 0.03$ & $24.93 \pm 0.07$ & $24.65 \pm 0.06$ & $24.46 \pm 0.11$ & $23.75 \pm 0.12$ & $23.78 \pm 0.17$ & $22.91 \pm 0.05$ & $22.98 \pm 0.25$ & $21.83 \pm 0.03$ & $20.93 \pm 0.01$ & $20.64 \pm 0.01$ & $20.48 \pm 0.04$ & $20.82 \pm 0.02$ \\
\hline & $>26.18$ & $>25.29$ & $68 \pm 0.18$ & $>26.53$ & $>26.32$ & $>25.53$ & $>24.68$ & $>24.29$ & $>24.88$ & $>23.06$ & $>24.35$ & & $>24.09$ & $>22.44$ & $>23.38$ \\
\hline ALESS 88.5 & $>26.18$ & $>25.29$ & $11 \pm 0.26$ & $>26.53$ & $26.07 \pm 0.22$ & $>25.53$ & $>24.68$ & $>24.29$ & $23.77 \pm 0.10$ & $22.12 \pm 0.12$ & $22.31 \pm 0.04$ & $21.52 \pm 0.02$ & $21.17 \pm 0.02$ & $20.93 \pm 0.06$ & $21.27 \pm 0.03$ \\
\hline ALESS 88.11 & $25.03 \pm 0.10$ & $24.92 \pm 0.20$ & $17 \pm 0.02$ & $24.06 \pm 0.03$ & $.65 \pm 0.03$ & $23.50 \pm 0.05$ & $23.19 \pm$ & $23.08 \pm 0.09$ & $22.99 \pm 0.05$ & $>23.06$ & $22.06 \pm$ & $21.37 \pm$ & $21.21 \pm 0.02$ & $21.18=$ & $21.50 \pm$ \\
\hline ALESS 92.2 & $25.56 \pm 0.16$ & $>25.29$ & $.55 \pm 0.03$ & $25.28 \pm 0.09$ & $24.82 \pm 0.07$ & $24.42 \pm 0.10$ & $24.48 \pm$ & $23.63 \pm 0.15$ & $23.83 \pm 0.11$ & & $75 \pm 0.16$ & & $23.52 \pm 0.15$ & $>22$ & $>23$ \\
\hline ALESS 94.1 & $>26.18$ & $>25.29$ & $\ldots$ & $26.03=$ & $25.92 \pm 0.19$ & & $>24.68$ & $>24.29$ & $>24.88$ & $>23.06$ & $.33 \pm$ & .03 & $21.66 \pm 0.03$ & $21.33=$ & $21.47 \pm 0.04$ \\
\hline ALESS 98.1 & & $>25.2$ & $\ldots$ & $>26$. & $>26.32$ & & $24.28 \pm 0.19$ & & $22.71 \pm 0.04$ & & $21.22 \pm 0.02$ & $9.86 \pm 0.00$ & $19.47 \pm 0.00$ & $19.59=$ & $19.91 \pm 0.01$ \\
\hline ALESS 99.1 & $>26.18$ & $>25.29$ & $28.10 \pm 0.26$ & $>26.53$ & $>26.32$ & $>25.53$ & $>24.68$ & $>24.29$ & $>24.88$ & $>23.06$ & $>24.35$ & $>24.45$ & $>24.09$ & $>22.44$ & $>23.38$ \\
\hline S 102.1 & & & $\ldots$ & $>26.53$ & $26.27 \pm 0.22$ & & & & $2.79 \pm 0.19^{* *}$ & $\ldots$ & $21.07 \pm$ & $20.07 \pm 0.00$ & $19.78 \pm 0.01$ & $19.77 \pm 0.02$ & $20.56 \pm 0.02$ \\
\hline ALESS 103.3 & $>26.18$ & $>25$ & $\ldots$ & $>26.53$ & $>26.32$ & $>25.53$ & $>24.6$ & $>24$. & $>23.22^{* *}$ & $\ldots$ & $>22.41^{* *}$ & $>24.45$ & $>24.09$ & $>22.44$ & $>23.38$ \\
\hline & & & $\ldots$ & $25.63 \pm 0.12$ & $24.62 \pm 0.06$ & $23.66 \pm 0.05$ & $22.61 \pm$ & $22.61 \pm$ & & $\ldots$ & & $20.49 \pm$ & $20.71 \pm 0.01$ & $20.77 \pm 0.05$ & $20.89 \pm 0.02$ \\
\hline ALESS 107.3 & $>26.18$ & $>25.29$ & $\ldots$ & $25.82 \pm 0.15$ & $25.52 \pm 0.14$ & $25.52 \pm 0.26$ & $>24.68$ & $>24.29$ & $24.44 \pm 0.18$ & $\ldots$ & 0.23 & $>24.45$ & $>24.09$ & $>22.44$ & $>23.38$ \\
\hline & $\ldots$ & $\ldots$ & $\ldots$ & $\ldots$ & $\ldots$ & $\ldots$ & $\ldots$ & $\ldots$ & $>24.88$ & $\ldots$ & & & $22.04 \pm 0.04$ & $21.53 \pm 0.10$ & $21.32 \pm 0.04$ \\
\hline $\mathrm{ALI}$ & $\ldots$ & $\ldots$ & $\ldots$ & $\ldots$ & $\ldots$ & $\ldots$ & $\ldots$ & $\ldots$ & $>24.88$ & $\ldots$ & $>24.35$ & $9 \pm 0.04$ & $23.01 \pm 0.10$ & $>22.44$ & $>23.38$ \\
\hline SS 112.1 & & $\ldots$ & $26.79 \pm 0.08$ & $\ldots$ & & & $\ldots$ & $\ldots$ & & $\ldots$ & $\ldots$ & $20.56 \pm 0.01$ & $20.22 \pm 0.01$ & $20.03 \pm 0.03$ & $20.66 \pm 0.02$ \\
\hline SS 114.1 & $>26.18$ & $>25.29$ & $>28.14$ & $>26.53$ & $>26.32$ & $>25.53$ & $>24.68$ & $>24.29$ & $>24.88$ & $>23.06$ & $>24.35$ & $3.99 \pm 0.16$ & $23.21 \pm 0.11$ & $>22.44$ & $>23.38$ \\
\hline ALESS 114.2 & $24.83 \pm 0.08$ & $24.87 \pm 0.19$ & $24.78 \pm 0.01$ & $24.24 \pm 0.04$ & $23.93 \pm 0.03$ & $23.56 \pm 0.05$ & $22.73 \pm 0.05$ & $22.61 \pm 0.06$ & $21.21 \pm 0.01$ & $20.58 \pm 0.03$ & $20.37 \pm 0.01$ & $19.56 \pm 0.00$ & $19.28 \pm 0.00$ & $19.46 \pm 0.02$ & $19.70 \pm 0.01$ \\
\hline ALESS $116.1^{\mathrm{a}}$ & $>26.18$ & $>25.29$ & $>28.14$ & $>26.53$ & $>26.32$ & $>25.53$ & $>24.68$ & & $>24.88$ & $>23.06$ & $.01 \pm 0.20$ & $23.52 \pm 0.11$ & $22.83 \pm 0.08$ & $>22.44$ & $22.49 \pm 0.10$ \\
\hline ALESS 116.2 & $>26.18$ & $>25.29$ & $>28.14$ & $>26.53$ & $>26.32$ & $>25.53$ & $>24.68$ & $>24.29$ & $>24.88$ & $>23.06$ & $23.85 \pm 0.17$ & $22.86 \pm 0.06$ & $22.22 \pm 0.05$ & $21.92 \pm 0.14$ & $21.87 \pm 0.06$ \\
\hline ALESS 118.1 & 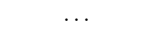 & 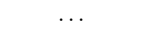 & $\ldots$ & $\ldots$ & $\ldots$ & $\ldots$ & $\ldots$ & $\ldots$ & $23.42 \pm 0.0$ & $\ldots$ & $22.67 \pm 0.06$ & $21.84 \pm 0.02$ & $21.29 \pm 0.02$ & $21.07 \pm 0.07$ & $21.35 \pm 0.04$ \\
\hline ALESS 119.1 & $>26.18$ & $>25.29$ & $\ldots$ & $>26.53$ & $.82 \pm 0.18$ & $25.17 \pm 0.20$ & $>24.68$ & $1.04 \pm 0.22$ & $24.20 \pm 0.20^{*}$ & $\ldots$ & $23.41 \pm 0.15^{*}$ & $22.78 \pm 0.06$ & $22.19 \pm 0.05$ & $21.66 \pm 0.11$ & $21.89 \pm 0.06$ \\
\hline ALESS 122.1 & $24.34 \pm 0.05$ & $24.31 \pm 0.12$ & $24.22 \pm 0.0$ & $23.49 \pm 0.02$ & $23.16 \pm 0.02$ & $22.96 \pm 0.03$ & $22.62 \pm 0.04$ & $22.55 \pm 0.06$ & $1.49 \pm 0.01$ & $\ldots$ & $20.68 \pm 0.01$ & $9.88 \pm 0.00$ & $19.51 \pm 0.00$ & $19.19 \pm 0.01$ & $19.27 \pm 0.01$ \\
\hline ALESS $124.1^{\mathrm{a}}$ & & & $>28.14$ & & $>26.32$ & & $>24.68$ & $>24.29$ & $24.63 \pm 0.22$ & $>23.06$ & $23.73 \pm 0.16$ & $21.92 \pm 0.03$ & $21.49 \pm 0.02$ & $21.44 \pm 0.09$ & $21.18 \pm 0.03$ \\
\hline ALESS 124.4 & & $>25$ & $>28.14$ & $>26.53$ & $25.96 \pm 0.20$ & & & 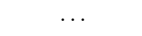 & $>24.88$ & $>23.06$ & $>24.35$ & $>24.45$ & $>24.09$ & $>22.44$ & $>23.38$ \\
\hline ALESS 126.1 & $>26.18$ & $>25.29$ & $26.68 \pm 0.08$ & $26.11 \pm 0.19$ & $26.31 \pm 0.26$ & $>25.53$ & $>24.68$ & - & $23.79 \pm 0.10$ & $22.33 \pm 0.14$ & $22.28 \pm 0.04$ & $20.95 \pm 0.01$ & $20.74 \pm 0.01$ & $20.86 \pm 0.06$ & $21.42 \pm 0.04$ \\
\hline
\end{tabular}

Notes. $3 \sigma$ upper limits are presented for non-detections, and the entry is left blank where a source is not covered by available imaging.

a Source is within 4" of a $3.6 \mu \mathrm{m}$ source of comparable or greater flux.

${ }^{\text {b }}$ We measure $J$ and $K_{S}$ photometry from three imaging surveys, but quote a single value, in order of $3 \sigma$ detection limit (see Table 1).

* Photometry measured from HAWK-I imaging.

** Photometry measured from MUSYC imaging; otherwise, photometry measured from TENIS imaging.

(This table is also available in a machine-readable form in the online journal.) 


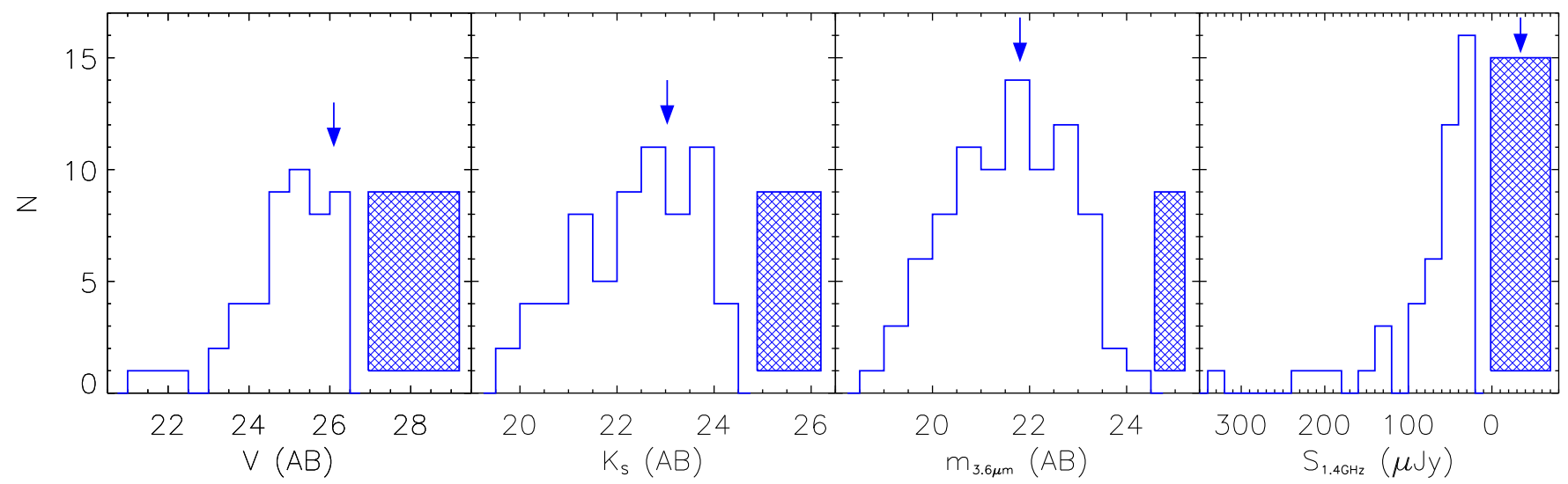

Figure 2. Apparent magnitude distributions of ALESS SMGs in the $V, K_{s}$, and IRAC $3.6 \mu \mathrm{m}$ bands, along with the flux density distribution at $1.4 \mathrm{GHz}$. On each panel a checked region indicates the undetected sources (see Table 1 for magnitude limits). The median SMG $V, K_{S}$, and $3.6 \mu$ m band magnitudes, including non-detections, are $V=26.09 \pm 0.19, K_{S}=23.0 \pm 0.3$, and $m_{3.6}=21.80 \pm 0.17$, respectively, and we mark these on each panel with an arrow. The radio data reach a depth of $19.5 \mu \mathrm{Jy}$ at its deepest ( $3 \sigma$ detection limit); however, only $45 \%$ of the ALESS SMGs are detected at this level, and the median $1.4 \mathrm{GHz}$ flux for SMGs is thus constrained at $\lesssim 19.5 \mu \mathrm{Jy}$.

(A color version of this figure is available in the online journal.)

\section{PHOTOMETRIC REDSHIFTS}

The first step in our analysis is to derive photometric redshifts for the ALESS SMGs in our sample, and so determine the first photometric distribution for a large submm-identified population of SMGs. To derive photometric redshifts, we use the SED fitting code HYPERZ (Bolzonella et al. 2000), which computes the $\chi^{2}$ statistic for a set of model SEDs to the observed photometry. In the case of non-detections we adopt a flux of zero during the SED fitting, but with an uncertainty equal to the $1 \sigma$ limiting magnitude in that filter. The model SEDs are characterized by a star formation history (SFH) and parameterized by age, reddening, and redshift. HYPERZ returns the best-fit parameters for the model SED corresponding to the lowest $\chi^{2}$. We use the spectral templates of Bruzual \& Charlot (2003), with solar metallicities, and consider four SFHs: a single burst (B), constant star formation (C), and two exponentially decaying SFHs with timescales of $1 \mathrm{Gyr}(\mathrm{E})$ and $5 \mathrm{Gyr}(\mathrm{Sb})$. Redshifts from $z=0$ to 7 are considered, and we allow reddening $\left(A_{V}\right)$ in the range $0-5$, in steps of 0.1 , following the Calzetti et al. (2000) dust law. We also include the constraint that the age of the galaxy must be less than the age of universe. Finally, we follow the same prescription as Wardlow et al. (2011) for handling of Ly $\alpha$ absorption in HYPERZ; the strength of the intergalactic absorption is increased, but we also allow a wider range of possible optical depths (see Wardlow et al. 2011).

\subsection{Training Sample}

Before deriving photometric redshifts for the ALESS SMGs, we first calibrate our photometry to the template SEDs used in the photometric redshift calculation. To do so, we use SEXTRACTOR to create a $3.6 \mu \mathrm{m}$ selected catalog designed to test the reliability of our photometric redshifts against archival spectroscopic surveys. The spectroscopic sample is collated from a wide range of sources (Cristiani et al. 2000; Croom et al. 2001; Cimatti et al. 2002; Teplitz et al. 2003; Bunker et al. 2003; Le Fèvre et al. 2004; Zheng et al. 2004; Szokoly et al. 2004; Strolger et al. 2004; Stanway et al. 2004; van der Wel et al. 2005; Mignoli et al. 2005; Daddi et al. 2005; Doherty et al. 2005; Ravikumar et al. 2007; Vanzella et al. 2008; Kriek et al. 2008; Popesso et al. 2009; Treister et al. 2009; Balestra et al. 2010; Silverman et al. 2010; Casey et al. 2011; Cooper et al. 2012; Bonzini et al. 2012; Swinbank et al. 2012; S. Koposov et al. in preparation; A. L. R. Danielson et al. in preparation), yielding 5942 spectroscopic redshifts with a median $z_{\text {spec }}=0.67$, an interquartile range of $0.45-0.85$, and 1077 galaxies at $z>1$. We measure photometry for these sources in the same manner as the ALESS SMGs (see Section 2.2.1). For reference, the spectroscopic sample has 10-90 percentile magnitude ranges of $V=21.6-24.4$, and $m_{3.6}=19.3-22.8$.

To test for small discrepancies between the observed photometry and the template SEDs, we run HYPERZ on our training set of 5942 galaxies with spectroscopic redshifts, fixing the redshift to the spectroscopic value. We then measure the offset between the observed photometry and that predicted from the best-fit model SED. We apply the measured offset to the observed photometry and then repeat the procedure for three iterations. After the final iteration we derive, and apply, significant offsets in the MUSYC $U$ $(-0.16), U_{38}(-0.12)$, MUSYC $J(-0.10), H(-0.14)$, HAWK $K_{S}(-0.10)$, TENIS $K(0.10), 5.8 \mu \mathrm{m}(0.19)$, and $8.0 \mu \mathrm{m}(0.40)$ photometry. Offsets in the remaining bands are $<0.06$, and the typical uncertainty is \pm 0.02 . The largest offset is an excess in the IRAC $8.0 \mu \mathrm{m}$, which may be due to a hot dust component in the SEDs that is not included in the HYPERZ templates. We test whether the $8.0 \mu \mathrm{m}$ data drive systematic offsets at other wavelengths by omitting the IRAC 5.8 and $8.0 \mu \mathrm{m}$ data and repeating the procedure, but we find that the magnitude offsets are consistent with those determined when these wavebands are included.

To determine the accuracy of our photometric redshifts, we initially compare the results for the 5942 galaxies in the ECDFS with spectroscopic redshifts. We calculate $\Delta z=$ $z_{\text {spec }}-z_{\text {phot }}$ for each galaxy and plot the histogram of $\Delta z / 1+z_{\text {spec }}$ in Figure 3. We find excellent agreement between the photometric and spectroscopic redshifts, measuring a median $\Delta z /\left(1+z_{\text {spec }}\right)=0.011 \pm 0.002$, with a $1 \sigma$ dispersion of 0.057 and a normalized median absolute deviation (NMAD) of $\sigma_{\mathrm{NMAD}}=1.48 \times \operatorname{median}\left(|\Delta z-\operatorname{median}(\Delta z)| / 1+z_{\mathrm{spec}}\right)=$ $0.073 .^{18}$

\footnotetext{
18 We also derived photometric redshifts for our training sample using the SED fitting code EAZY (Brammer et al. 2008). We find that the photometric redshifts derived by EAZY are comparable with those from HYPERZ, with a median $\Delta z / 1+z_{\text {spec }}=0.020 \pm 0.006$, consistent with Dahlen et al. (2013), who find comparable performance between photometric redshift estimation codes.
} 


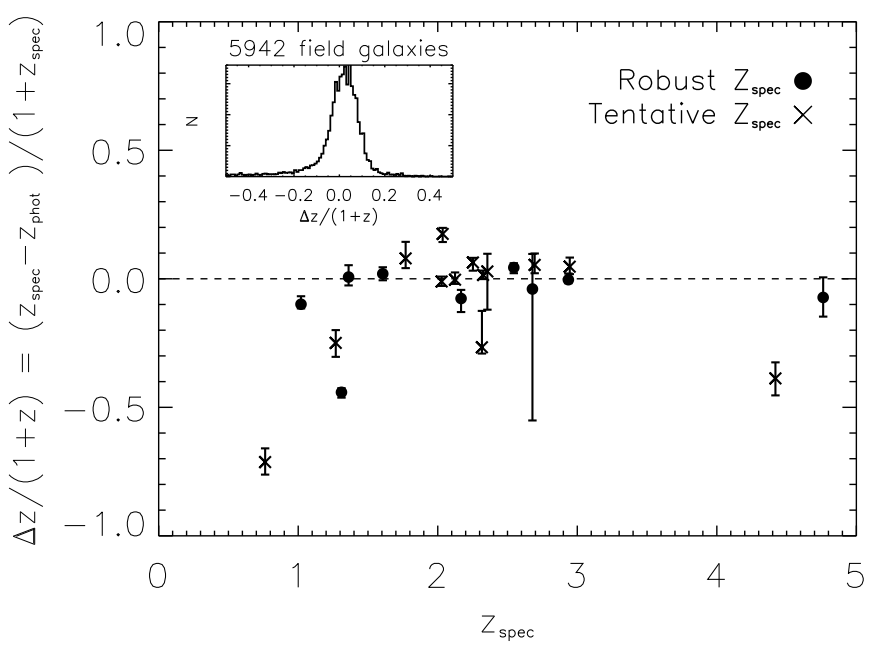

Figure 3. Comparison of the photometric redshifts derived in this work, to spectroscopically confirmed redshifts drawn from the literature and from our redshift follow-up of the original LESS sources (zLESS; A. L. R. Danielson et al. in preparation). The inset shows the distribution of $\Delta z /\left(1+z_{\mathrm{spec}}\right)$ for a $3.6 \mu \mathrm{m}$ selected training sample with spectroscopic redshifts. For the field sample we find good agreement between the photometric and spectroscopic redshifts, with a median $\Delta z /\left(1+z_{\text {spec }}\right)$ of $0.011 \pm 0.002$ and a $1 \sigma$ dispersion of 0.06. In the main panel we compare the photometric redshifts for 22 ALESS SMGs with confirmed spectroscopic redshifts. We again find good agreement, with a median $\Delta z /\left(1+z_{\text {spec }}\right)$ of $-0.004 \pm 0.026$. We identify spectroscopic redshifts as robust where they are calculated from multiple strong emission lines, and tentative where multiple weak lines or single line IDs are used. We identify three outliers, at $\left|\Delta z /\left(1+z_{\text {spec }}\right)\right|>0.3$. Of the three sources only one, ALESS 66.1, has a robust spectroscopic redshift and is an optically bright QSO. The remaining two sources have spectroscopic redshifts drawn from single line identifications.

Previous studies indicate that the majority of the ALESS SMGs lie at $z>1.0$ (see Wardlow et al. 2011), and so we also investigate the accuracy of our photometric redshifts limiting just to this redshift range. For the $z>1.0$ sources in the training sample the median $\Delta z /\left(1+z_{\mathrm{spec}}\right)$ is $0.033 \pm 0.005$, marginally higher than for the training sample as a whole. We define catastrophic failures as sources with $\Delta z /\left(1+z_{\text {spec }}\right)>0.3$, and we find that the failure rate for the 1077 sources at $z>1.0$ is $4 \%$. Importantly, the $z>1.0$ training sample has a median $3.6 \mu \mathrm{m}$ magnitude of $m_{3.6}=21.2 \pm 0.1$, which is similar to the median $3.6 \mu \mathrm{m}$ magnitude of the ALESS sample, $m_{3.6}=21.8 \pm 0.2$.

Although HYPERZ returns a best-fit model and $1 \sigma$ error, for our sample of field galaxies we determine that the HYPERZ "99\%" confidence intervals provide the best estimate of the redshift error, yielding $\sim 68 \%$ agreement between the photometric and spectroscopic redshifts at $1 \sigma$, and so we adopt these as our $1 \sigma$ error estimates (see also Luo et al. 2010; Wardlow et al. 2011).

\subsection{ALESS Photometric Redshifts}

Before deriving the redshift distribution for all ALESS SMGs, we next make use of the existing spectroscopy of ALESS sources to test the reliability of our photometric redshifts for SMGs. Combining our results with a small number from the literature, we have spectroscopic redshifts for 22 ALESS SMGs (Zheng et al. 2004; Kriek et al. 2008; Coppin et al. 2009; Silverman et al. 2010; Casey et al. 2011; Bonzini et al. 2012; Swinbank et al. 2012; A. L. R. Danielson et al. in preparation). We run HYPERZ on these SMGs to derive their photometric redshifts, and in Figure 3 we compare the spectroscopic results to our photometric redshifts (Figure 3) and find a median

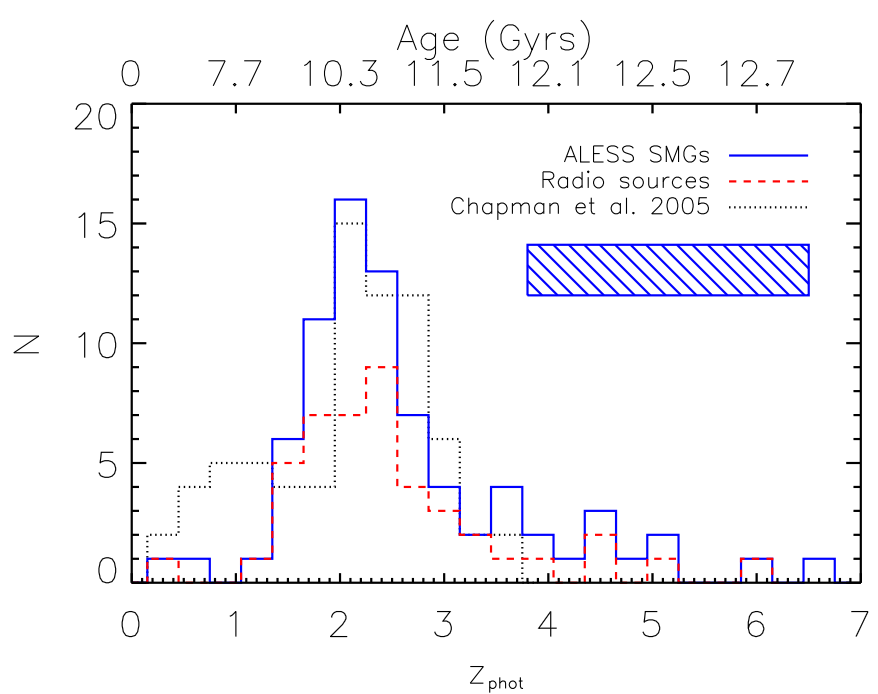

Figure 4. Photometric redshift distribution of ALESS SMGs with individually derived photometric redshifts. For comparison we show the spectroscopic redshift distribution from Chapman et al. (2005), a radio-selected sample of SMGs. We find that the ALESS SMGs lie at a median redshift of $z=2.3 \pm 0.1$, consistent with the result from Chapman et al. (2005). In contrast to Chapman et al. (2005), we do not find a significant number of SMGs at $z \lesssim 1$, and we identify a high-redshift tail at $z \gtrsim 3.5$, not seen in Chapman et al. (2005). The hatched box represents the area missing from the ALESS histogram due to 19 SMGs with insufficient photometry to derive photometric redshifts. In Section 3.2.3 we identify these sources as belonging to the high-redshift tail of the distribution (i.e., $z \gtrsim 3$ ). Including these 19 SMGs raises the median redshift to $z=2.5 \pm 0.2$; see Figure 12 .

(A color version of this figure is available in the online journal.)

$\Delta z /\left(1+z_{\text {spec }}\right)=-0.004 \pm 0.026$ and $\sigma_{\mathrm{NMAD}}=0.099$. The spectroscopically confirmed ALESS SMGs have a median redshift $z_{\text {spec }}=2.2 \pm 0.2$ and a median $3.6 \mu \mathrm{m}$ magnitude of $m_{3.6}=20.5 \pm 0.5$. Together with the results for the 5924 galaxies in the spectroscopic training sample, we can therefore be confident that the photometric redshifts we derive provide a reliable estimate of the SMG population.

\subsubsection{Reliability of SMG Redshifts}

Running HYPERZ on the photometry catalog of 77 ALESS SMGs, with detections in $>3$ wavebands, we derive a median photometric redshift of $z_{\text {phot }}=2.3 \pm 0.1$, with a tail to $z \sim 6$ (Figure 4) and a $1 \sigma$ spread of $z_{\text {phot }}=1.8-3.5$. In Table 3 we provide the redshifts for individual sources. We note that we will return to discussing the 19 SMGs detected in fewer than four wavebands in Section 3.2.3. We caution that five SMGs (ALESS 5.1, 6.1, 57.1, 66.1, and 75.1) have best-fit solutions with anomalously high values of $\chi_{\text {red }}^{2}(>10)$. We inspect the photometry for each of these and find that ALESS 57.1, 66.1, and 75.1 have an $8.0 \mu \mathrm{m}$ excess consistent with obscured AGN activity (ALESS 66.1 is an optically identified QSO, ALESS 57.1 is an X-ray-detected SMG, and ALESS 75.1 has excess radio emission consistent with AGN activity; Wang et al. 2013). As we do not include AGN templates in our model SEDs, it is unsurprising that we find poor agreement for these sources. For the remaining two sources, the photometry of ALESS 5.1 is dominated by a large nearby galaxy, while ALESS 6.1 is a potential lensed source; the $870 \mu \mathrm{m}$ emission is offset by $\sim 1^{\prime \prime} .5$ from a bright optical source at $z_{\text {phot }} \sim 0.4$. We therefore advise that the photometric redshifts for ALESS 5.1 and 6.1 are treated with caution, and we highlight these SMGs in Table 3. 
Table 3

Derived Properties

\begin{tabular}{|c|c|c|c|c|c|c|c|c|}
\hline ID & $\begin{array}{l}\text { R.A. } \\
\text { (J2000) }\end{array}$ & $\begin{array}{c}\text { Decl. } \\
(\mathrm{J} 2000)\end{array}$ & $z_{\text {phot }}$ & $z_{\text {spec }}$ & $\chi_{\text {red }}^{2}$ & $\begin{array}{c}\text { Filters } \\
\text { (Det [Obs]) }\end{array}$ & $\begin{array}{c}M_{H} \\
(\mathrm{AB})\end{array}$ & $\begin{array}{c}M / L_{H} \\
\left(M_{\odot} L_{\odot}^{-1}\right) \\
\end{array}$ \\
\hline ALESS 001.1 & $03: 33: 14.46$ & $-27: 56: 14.5$ & $4.34_{-1.43}^{+2.66}$ & & 0.91 & $5[15]$ & -24.90 & 0.14 \\
\hline ALESS 001.2 & 03:33:14.41 & $-27: 56: 11.6$ & $4.65_{-1.02}^{+2.34}$ & & 1.04 & $5[15]$ & -24.79 & 0.29 \\
\hline ALESS 001.3 & 03:33:14.18 & $-27: 56: 12.3$ & $2.85_{-0.30}^{+0.20}$ & & 3.78 & $5[15]$ & -23.83 & 0.29 \\
\hline ALESS 002.1 & 03:33:02.69 & $-27: 56: 42.8$ & $1.96_{-0.20}^{+0.27}$ & & 1.39 & $8[14]$ & -23.24 & 0.17 \\
\hline ALESS 003.1 & $03: 33: 21.50$ & $-27: 55: 20.3$ & $3.90_{-0.59}^{+0.50}$ & & 0.68 & $5[14]$ & -25.51 & 0.20 \\
\hline ALESS $005.1^{\mathrm{a}}$ & 03:31:28.91 & $-27: 59: 09.0$ & $2.86_{-0.04}^{+0.05}$ & & 14.76 & 13 [13] & -25.71 & 0.04 \\
\hline ALESS $006.1^{\mathrm{a}}$ & 03:32:56.96 & $-28: 01: 00.7$ & $0.45_{-0.04}^{+0.06}$ & & 17.54 & 15 [15] & -21.95 & 0.25 \\
\hline ALESS 007.1 & 03:33:15.42 & $-27: 45: 24.3$ & $2.50_{-0.16}^{+0.12}$ & & 8.32 & 14 [15] & -25.96 & 0.04 \\
\hline ALESS 009.1 & 03:32:11.34 & $-27: 52: 11.9$ & $4.50_{-2.33}^{+0.54}$ & & 0.22 & $5[14]$ & -25.98 & 0.25 \\
\hline ALESS 010.1 & 03:32:19.06 & $-27: 52: 14.8$ & $2.02_{-0.09}^{+0.09}$ & & 9.89 & $12[13]$ & -23.57 & 0.15 \\
\hline ALESS 011.1 & 03:32:13.85 & $-27: 56: 00.3$ & $2.83_{-0.50}^{+1.88}$ & & 2.02 & $6[15]$ & -24.95 & 0.36 \\
\hline ALESS 013.1 & 03:32:48.99 & $-27: 42: 51.8$ & $3.25_{-0.46}^{+0.64}$ & & 2.12 & $8[15]$ & -25.05 & 0.11 \\
\hline ALESS 014.1 & 03:31:52.49 & $-28: 03: 19.1$ & $4.47_{-0.88}^{+2.54}$ & & 1.46 & $6[13]$ & -26.13 & 0.06 \\
\hline ALESS 015.1 & 03:33:33.37 & $-27: 59: 29.6$ & $1.93_{-0.33}^{+0.62}$ & & 0.43 & $4[13]$ & -23.94 & 0.21 \\
\hline ALESS 017.1 & 03:32:07.30 & $-27: 51: 20.8$ & $1.51_{-0.07}^{+0.10}$ & & 1.95 & 15 [15] & -24.46 & 0.18 \\
\hline ALESS 018.1 & 03:32:04.88 & $-27: 46: 47.7$ & $2.04_{-0.06}^{+0.10}$ & $2.25^{\mathrm{b}}$ & 5.44 & 14 [15] & -25.33 & 0.15 \\
\hline ALESS 019.1 & 03:32:08.26 & $-27: 58: 14.2$ & $2.41_{-0.11}^{+0.17}$ & & 8.40 & $7[14]$ & -23.93 & 0.15 \\
\hline ALESS 019.2 & 03:32:07.89 & $-27: 58: 24.1$ & $2.17_{-0.10}^{+0.09}$ & & 2.04 & $13[14]$ & -23.74 & 0.14 \\
\hline ALESS 022.1 & 03:31:46.92 & $-27: 32: 39.3$ & $1.88_{-0.23}^{+0.18}$ & & 3.50 & $6[11]$ & -25.04 & 0.22 \\
\hline ALESS 023.1 & 03:32:12.01 & $-28: 05: 06.5$ & $4.99_{-2.55}^{+2.01}$ & & 0.35 & $4[6]$ & -25.78 & 0.09 \\
\hline ALESS 025.1 & 03:31:56.88 & $-27: 59: 39.3$ & $2.24_{-0.17}^{+0.07}$ & & 1.65 & $12[14]$ & -25.03 & 0.17 \\
\hline ALESS 029.1 & 03:33:36.90 & $-27: 58: 09.3$ & $2.66_{-0.76}^{+2.94}$ & & 0.10 & $4[13]$ & -24.78 & 0.36 \\
\hline ALESS 031.1 & 03:31:49.79 & $-27: 57: 40.8$ & $2.89_{-0.41}^{+1.80}$ & & 1.09 & $5[13]$ & -24.62 & 0.12 \\
\hline ALESS 037.1 & $03: 33: 36.14$ & $-27: 53: 50.6$ & $3.53_{-0.31}^{+0.56}$ & & 2.07 & $9[12]$ & -25.73 & 0.04 \\
\hline ALESS 037.2 & 03:33:36.36 & $-27: 53: 48.3$ & $4.87_{-0.40}^{+0.22}$ & & 0.95 & $6[12]$ & -25.26 & 0.17 \\
\hline ALESS 039.1 & 03:31:45.03 & $-27: 34: 36.7$ & $2.44_{-0.23}^{+0.17}$ & & 9.14 & $11[13]$ & -24.74 & 0.04 \\
\hline ALESS 041.1 & 03:31:10.07 & $-27: 52: 36.7$ & $2.75_{-0.72}^{+4.25}$ & & 0.00 & $4[4]$ & -26.15 & 0.17 \\
\hline ALESS 043.1 & 03:33:06.64 & $-27: 48: 02.4$ & $1.71_{-0.12}^{+0.20}$ & & 4.33 & $7[14]$ & -23.85 & 0.22 \\
\hline ALESS 045.1 & $03: 32: 25.26$ & $-27: 52: 30.5$ & $2.34_{-0.67}^{+0.26}$ & & 0.32 & $5[14]$ & -24.77 & 0.36 \\
\hline ALESS 049.1 & 03:31:24.72 & $-27: 50: 47.1$ & $2.76_{-0.14}^{+0.11}$ & & 1.56 & $11[13]$ & -24.44 & 0.05 \\
\hline ALESS 049.2 & 03:31:24.47 & $-27: 50: 38.1$ & $1.47_{-0.10}^{+0.07}$ & & 3.99 & $12[13]$ & -23.55 & 0.04 \\
\hline ALESS 051.1 & 03:31:45.06 & $-27: 44: 27.3$ & $1.22_{-0.06}^{+0.03}$ & & 7.76 & 13 [15] & -24.36 & 0.25 \\
\hline ALESS 055.1 & 03:33:02.22 & $-27: 40: 35.4$ & $2.05_{-0.13}^{+0.15}$ & & 7.04 & $13[14]$ & -22.93 & 0.15 \\
\hline ALESS 055.5 & 03:33:02.35 & $-27: 40: 35.4$ & $2.35_{-0.13}^{+0.11}$ & & 6.89 & $12[14]$ & -22.97 & 0.15 \\
\hline ALESS 057.1 & 03:31:51.92 & $-27: 53: 27.1$ & $2.95_{-0.10}^{+0.05}$ & $2.94^{\mathrm{c}}$ & 17.28 & $11[15]$ & -24.91 & 0.15 \\
\hline ALESS 059.2 & 03:33:03.82 & $-27: 44: 18.2$ & $2.09_{-0.29}^{+0.78}$ & & 3.88 & $8[14]$ & -22.55 & 0.15 \\
\hline ALESS 061.1 & $03: 32: 45.87$ & $-28: 00: 23.4$ & $6.52_{-0.34}^{+0.36}$ & $4.44^{\mathrm{d}}$ & 3.97 & $7[13]$ & -25.61 & 0.05 \\
\hline ALESS 063.1 & 03:33:08.45 & $-28: 00: 43.8$ & $1.87_{-0.33}^{+0.10}$ & & 3.07 & $11[13]$ & -24.43 & 0.14 \\
\hline ALESS 066.1 & 03:33:31.93 & $-27: 54: 09.5$ & $2.33_{-0.04}^{+0.05}$ & $1.31^{\mathrm{e}}$ & 46.79 & $13[13]$ & -26.24 & 0.15 \\
\hline ALESS 067.1 & 03:32:43.20 & $-27: 55: 14.3$ & $2.14_{-0.09}^{+0.05}$ & $2.12^{\mathrm{f}}$ & 3.31 & $13[14]$ & -25.35 & 0.15 \\
\hline ALESS 067.2 & 03:32:43.02 & $-27: 55: 14.7$ & $2.05_{-0.16}^{+0.06}$ & & 7.42 & $12[14]$ & -23.91 & 0.05 \\
\hline ALESS 069.1 & 03:31:33.78 & $-27: 59: 32.4$ & $2.34_{-0.44}^{+0.27}$ & & 0.51 & $5[13]$ & -24.60 & 0.36 \\
\hline ALESS 070.1 & 03:31:44.02 & $-27: 38: 35.5$ & $2.28_{-0.06}^{+0.05}$ & & 2.47 & $14[14]$ & -25.37 & 0.15 \\
\hline ALESS 071.1 & 03:33:05.65 & $-27: 33: 28.2$ & $2.48_{-0.11}^{+0.21}$ & & 7.65 & $11[13]$ & -27.80 & 0.04 \\
\hline ALESS 071.3 & 03:33:06.14 & $-27: 33: 23.1$ & $2.73_{-0.25}^{+0.22}$ & & 2.87 & $6[13]$ & -22.24 & 0.15 \\
\hline ALESS 073.1 & 03:32:29.29 & $-27: 56: 19.7$ & $5.18_{-0.45}^{+0.43}$ & $4.76^{\mathrm{g}}$ & 2.00 & $8[14]$ & -25.61 & 0.07 \\
\hline ALESS 074.1 & 03:33:09.15 & $-27: 48: 17.2$ & $1.80_{-0.13}^{+0.13}$ & & 4.95 & $7[14]$ & -23.90 & 0.19 \\
\hline ALESS 075.1 & 03:31:27.19 & $-27: 55: 51.3$ & $2.39_{-0.06}^{+0.08}$ & & 41.20 & $13[13]$ & -25.97 & 0.05 \\
\hline ALESS $075.4^{\mathrm{a}}$ & 03:31:26.57 & $-27: 55: 55.7$ & $2.10_{-0.34}^{+0.29}$ & & 3.14 & $6[11]$ & -20.94 & 0.09 \\
\hline ALESS 079.1 & $03: 32: 21.14$ & $-27: 56: 27.0$ & $2.04_{-0.31}^{+0.63}$ & & 0.29 & $5[14]$ & -23.88 & 0.36 \\
\hline ALESS 079.2 & 03:32:21.60 & $-27: 56: 24.0$ & $1.55_{-0.18}^{+0.11}$ & & 2.42 & $13[14]$ & -24.56 & 0.18 \\
\hline ALESS 080.1 & 03:31:42.80 & $-27: 48: 36.9$ & $1.96_{-0.14}^{+0.16}$ & & 3.24 & $9[14]$ & -23.77 & 0.15 \\
\hline ALESS 080.2 & $03: 31: 42.62$ & $-27: 48: 41.0$ & $1.37_{-0.08}^{+0.17}$ & & 4.06 & $9[14]$ & -22.81 & 0.15 \\
\hline
\end{tabular}


Table 3

(Continued)

\begin{tabular}{|c|c|c|c|c|c|c|c|c|}
\hline ID & $\begin{array}{l}\text { R.A. } \\
\text { (J2000) }\end{array}$ & $\begin{array}{c}\text { Decl. } \\
\text { (J2000) }\end{array}$ & $z_{\text {phot }}$ & $z_{\text {spec }}$ & $\chi_{\mathrm{red}}^{2}$ & $\begin{array}{c}\text { Filters } \\
\text { (Det [Obs]) }\end{array}$ & $\begin{array}{l}M_{H} \\
(\mathrm{AB})\end{array}$ & $\begin{array}{c}M / L_{H} \\
\left(M_{\odot} L_{\odot}^{-1}\right) \\
\end{array}$ \\
\hline ALESS 082.1 & $03: 32: 54.00$ & $-27: 38: 14.9$ & $2.10_{-0.44}^{+3.27}$ & & 0.38 & $6[14]$ & -23.34 & 0.14 \\
\hline ALESS $083.4^{\mathrm{a}}$ & 03:33:08.71 & $-28: 05: 18.5$ & $0.57_{-0.50}^{+1.54}$ & & 0.07 & $4[4]$ & -21.51 & 0.11 \\
\hline ALESS 084.1 & $03: 31: 54.50$ & $-27: 51: 05.6$ & $1.92_{-0.07}^{+0.09}$ & & 3.71 & $14[14]$ & -24.11 & 0.15 \\
\hline ALESS 084.2 & 03:31:53.85 & $-27: 51: 04.3$ & $1.75_{-0.19}^{+0.08}$ & & 1.70 & $13[15]$ & -23.77 & 0.20 \\
\hline ALESS 087.1 & $03: 32: 50.88$ & $-27: 31: 41.5$ & $3.20_{-0.47}^{+0.08}$ & & 0.22 & $5[5]$ & -25.68 & 0.04 \\
\hline ALESS 088.1 & $03: 31: 54.76$ & $-27: 53: 41.5$ & $1.84_{-0.11}^{+0.12}$ & $1.27^{\mathrm{h}}$ & 3.04 & $14[15]$ & -24.11 & 0.15 \\
\hline ALESS 088.5 & 03:31:55.81 & $-27: 53: 47.2$ & $2.30_{-0.50}^{+0.11}$ & & 3.69 & $9[15]$ & -24.34 & 0.25 \\
\hline ALESS 088.11 & 03:31:54.95 & $-27: 53: 37.6$ & $2.57_{-0.12}^{+0.04}$ & & 8.73 & $14[15]$ & -24.32 & 0.07 \\
\hline ALESS 092.2 & $03: 31: 38.14$ & $-27: 43: 43.4$ & $1.90_{-0.75}^{+0.28}$ & & 2.66 & $11[14]$ & -21.17 & 0.04 \\
\hline ALESS 094.1 & 03:33:07.59 & $-27: 58: 05.8$ & $2.87_{-0.64}^{+0.37}$ & & 3.98 & $7[14]$ & -24.46 & 0.15 \\
\hline ALESS 098.1 & 03:31:29.92 & $-27: 57: 22.7$ & $1.63_{-0.09}^{+0.17}$ & $1.48^{\mathrm{b}}$ & 2.65 & $7[12]$ & -24.97 & 0.20 \\
\hline ALESS 102.1 & 03:33:35.60 & $-27: 40: 23.0$ & $1.76_{-0.18}^{+0.16}$ & & 4.42 & 7 [13] & -24.81 & 0.27 \\
\hline ALESS 107.1 & $03: 31: 30.50$ & $-27: 51: 49.1$ & $3.75_{-0.08}^{+0.09}$ & & 3.55 & $11[13]$ & -25.49 & 0.04 \\
\hline ALESS 107.3 & $03: 31: 30.72$ & $-27: 51: 55.7$ & $2.12_{-0.81}^{+1.54}$ & & 1.91 & $5[13]$ & -20.89 & 0.11 \\
\hline ALESS 110.1 & $03: 31: 22.66$ & $-27: 54: 17.2$ & $2.55_{-0.50}^{+0.70}$ & & 0.78 & $4[6]$ & -24.01 & 0.36 \\
\hline ALESS 112.1 & $03: 32: 48.86$ & $-27: 31: 13.3$ & $1.95_{-0.26}^{+0.15}$ & & 2.73 & $5[5]$ & -24.67 & 0.22 \\
\hline ALESS 114.2 & 03:31:51.11 & $-27: 44: 37.3$ & $1.56_{-0.07}^{+0.07}$ & $1.61^{\mathrm{h}}$ & 3.12 & 15 [15] & -25.02 & 0.17 \\
\hline ALESS 116.1 & $03: 31: 54.32$ & $-27: 45: 28.9$ & $3.54_{-0.87}^{+1.47}$ & & 0.82 & $4[15]$ & -23.84 & 0.25 \\
\hline ALESS 116.2 & 03:31:54.44 & $-27: 45: 31.4$ & $4.02_{-2.19}^{+1.19}$ & & 0.50 & $5[15]$ & -24.65 & 0.04 \\
\hline ALESS 118.1 & 03:31:21.92 & $-27: 49: 41.4$ & $2.26_{-0.23}^{+0.50}$ & & 3.85 & $6[6]$ & -24.15 & 0.04 \\
\hline ALESS 119.1 & $03: 32: 56.64$ & $-28: 03: 25.2$ & $3.50_{-0.35}^{+0.95}$ & & 3.41 & $9[13]$ & -24.42 & 0.05 \\
\hline ALESS 122.1 & $03: 31: 39.54$ & $-27: 41: 19.7$ & $2.06_{-0.06}^{+0.05}$ & $2.03^{\mathrm{i}}$ & 6.08 & $14[14]$ & -25.53 & 0.15 \\
\hline ALESS 124.1 & 03:32:04.04 & $-27: 36: 06.4$ & $6.07_{-1.16}^{+0.94}$ & & 0.80 & $6[15]$ & -26.22 & 0.16 \\
\hline ALESS 126.1 & 03:32:09.61 & $-27: 41: 07.7$ & $1.82_{-0.08}^{+0.28}$ & & 7.42 & $10[14]$ & -23.93 & 0.15 \\
\hline
\end{tabular}

Notes.

a As discussed in Section 3.2.1, these SMGs are potential gravitational lenses or have significantly contaminated photometry. We advise that the photometric redshifts for these SMGs are treated with extreme caution.

${ }^{\mathrm{b}}$ Casey et al. (2011).

${ }^{c}$ Zheng et al. (2004).

${ }^{\mathrm{d}}$ Swinbank et al. (2012).

e Silverman et al. (2010).

${ }^{\mathrm{f}}$ Kriek et al. (2008).

g Coppin et al. (2009).

${ }^{\text {h }}$ Coppin et al. (2012); A. L. R. Danielson et al., in preparation.

${ }^{i}$ Bonzini et al. (2012).

(This table is also available in a machine-readable form in the online journal.)

For six ALESS SMGs we derive photometric redshifts from detections in only four wavebands, our enforced minimum (although we note that the SED fit is constrained by sensitive upper limits in the remaining wavebands). To test if this introduces a bias in our following analysis, we take the photometry for 37 SMGs in our sample detected in $>8$ wavebands and make each source intrinsically fainter until only four of the photometry points remain above our detection limits. We then repeat the SED fitting procedure on these "faded" SMGs. We find a median offset in $\left(z_{4}-z_{\mathrm{All}}\right) /\left(1+z_{\mathrm{All}}\right)=-0.098 \pm 0.050$ and agreement at $3 \sigma$ for all sources. Crucially, while we find increased scatter between the original and faded photometric redshifts, and larger associated uncertainties, we do not find any bias toward higher, or lower, redshifts. ${ }^{19}$

\footnotetext{
19 We also test the likely effect of emission lines on the SED fitting using a young/blue template, with emission lines of similar equivalent width to SMGs (Swinbank et al. 2004), provided with the EAZY SED fitting code (Brammer et al. 2008). We run HYPERZ on the ALESS SMGs, using both the emission-line template and the same template with all emission lines removed. The resulting
}

Five SMGs in our sample are covered by IRAC imaging alone. To test the reliability of redshifts for ALESS SMGs derived from such photometry, we take the same subsample of 37 SMGs, remove all other photometric data, including upper limits, and repeat the SED fitting. We find a median offset in $\left(z_{\text {IRAC }}-z_{\text {All }}\right) /\left(1+z_{\text {All }}\right)=0.015 \pm 0.031$ and agreement at $3 \sigma$ for 36/37 SMGs. If we restrict our comparison to the ALESS SMGs with spectroscopic redshifts, then we find $\left(z_{\text {IRAC }}-z_{\text {spec }}\right) /$ $\left(1+z_{\text {spec }}\right)=-0.09 \pm 0.13$, with a median error on each photometric redshift of $\sigma_{\mathrm{z}}=0.6$. We note that if we only use three photometric data points in the SED fitting, then the photometric redshifts are unconstrained, with a median $1 \sigma$ error of $\sigma_{\mathrm{z}}=2.0$. We therefore can be confident in the

photometric redshifts are in agreement to within $\Delta z /(1+z)=0.000 \pm 0.001$ We observe a small increase in scatter at $z \sim 2.5$, which we attribute to $\mathrm{H} \alpha$ falling in/out of the $K_{s}$ band. The effect is small and over the redshift range $z=2.2-2.8$, and we measure $\Delta z /(1+z)=0.009 \pm 0.009$. Due to the modest magnitude of the effect of $\mathrm{H} \alpha$ on the photometric redshifts, we do not make any attempt to correct for it in our SED fitting. 

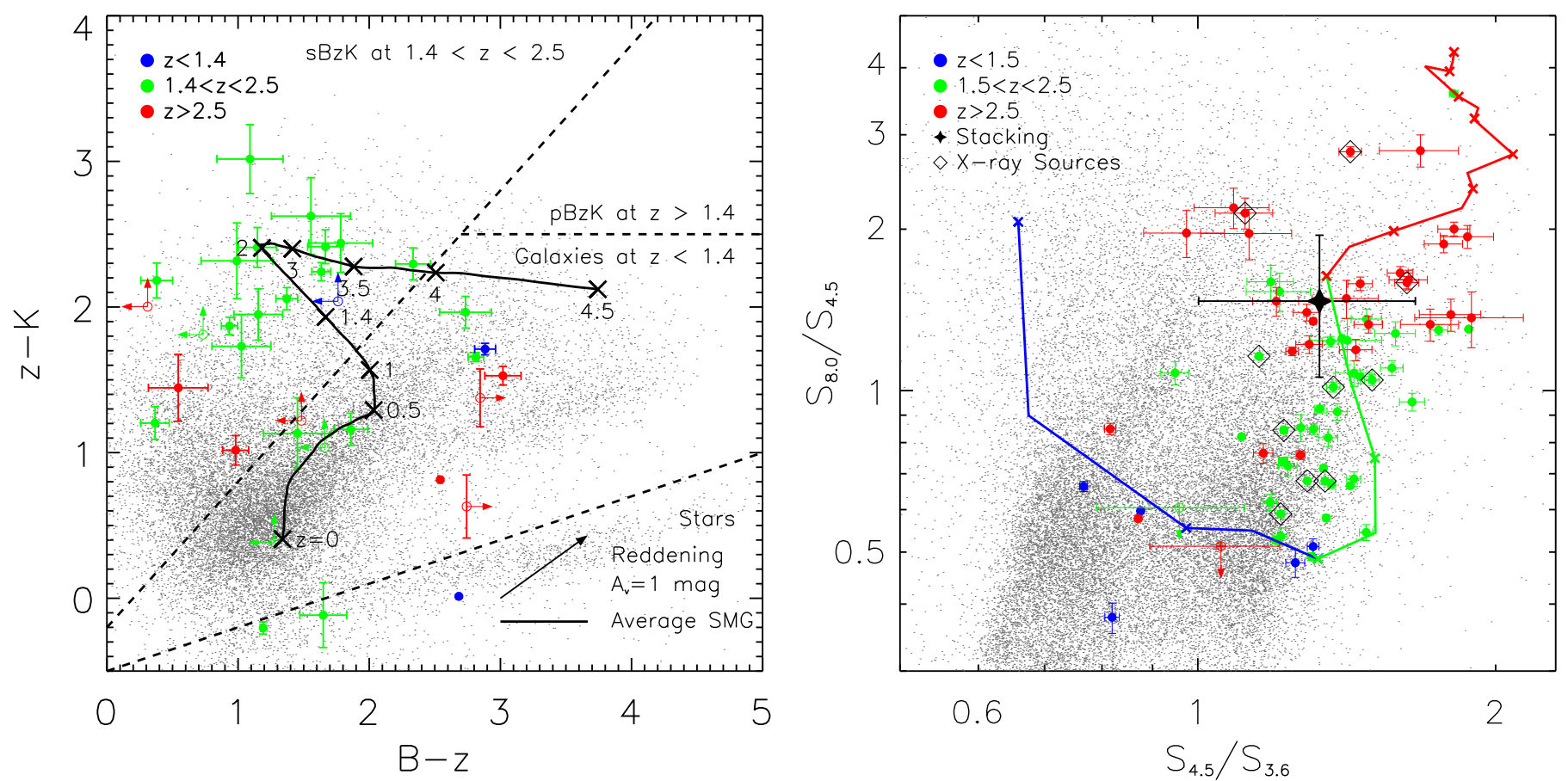

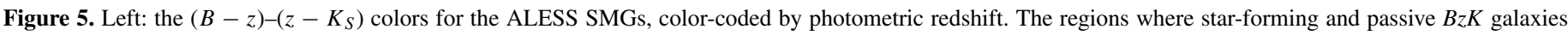

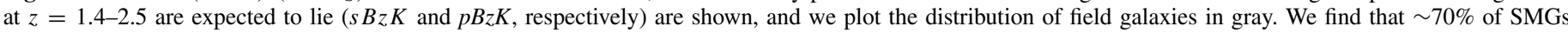

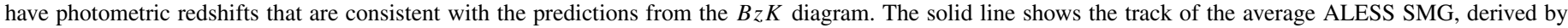

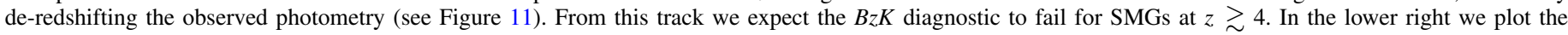

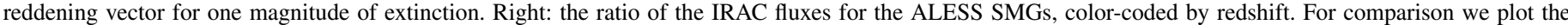

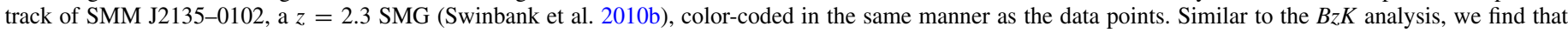

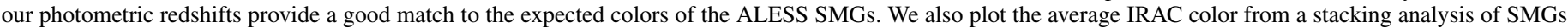

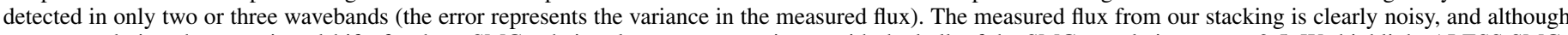

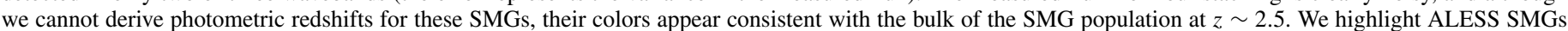

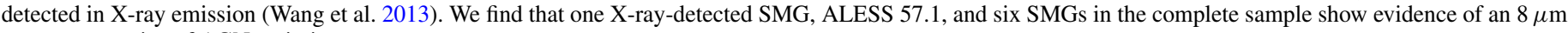
excess suggestive of AGN emission.

(A color version of this figure is available in the online journal.)

reliability of photometric redshifts derived from detections in four photometric bands and adopt this limit throughout our analysis.

Finally, we investigate the effect of source blending on our results. We re-measure aperture photometry for all of the ALESS SMGs, in the same manner described in Section 2.2.1, but with a $2^{\prime \prime}$ diameter aperture across all wavelengths. A smaller aperture means that the effects of blending are reduced, especially in the IRAC data. We repeat the SED fitting procedure described in Section 3, to derive photometric redshifts from the new, small-aperture photometry. Considering all ALESS SMGs, we find good agreement between the photometric redshifts, with $\left(z_{\text {Original }}-z_{\text {SmallAperture }}\right) /\left(1+z_{\text {All }}\right)=-0.012 \pm 0.009$. Of the 12 SMGs we flag as blended with a nearby bright IRAC source (see Table 2), 9 have a photometric redshift derived from photometry measured in a smaller aperture, which is consistent with the original redshift to within $1 \sigma$. Of the remaining three SMGs, ALESS 5.1 has been discussed already as a possible lens system, and two other sources, ALESS 75.4 and 83.4, are not detected in $>3$ wavebands in the smaller apertures. We highlight these SMGs in Table 3 and note that their redshifts should be treated with caution. In Figure 9 we highlight these three SMGs, along with ALESS 6.1, another potential lens system, as having suspicious photometry. We conclude that blending of sources does not have a significant effect on the bulk of the redshifts we derive.

\subsubsection{Redshift Indicators}

A number of color-color diagnostics have been suggested to identify star-forming galaxies. We consider three of these as simple tests of the reliability of our photometric redshifts. The first we consider is the $B z K$ diagram, which has been proposed as a tool to separate star-forming and passive galaxies at $z \sim 1.4-2.5$, by means of identifying the Balmer/4000 $\AA$ break. In Figure 5 we show the $B z K$ diagram for the ALESS SMGs with suitable photometric detections. We find that within the photometric errors $65 \%$ are correctly identified as starforming at $z>1.4$ and $25 \%$ are incorrectly classed as lying at $z<1.4$. One ALESS SMG is correctly classified as a galaxy at $z<1.4$, and no ALESS SMGs are classed as passive galaxies at $z>1.4$. Three ALESS SMGs have $B z K$ colors consistent with stars, one of which, ALESS 66.1, is an optically identified QSO. We caution that half of the ALESS SMGs incorrectly classified as galaxies at $z<1.4$ have photometric redshifts greater than the upper range of the $B z K$ diagnostic, i.e., $z>2.5$. We plot the SED for the composite ALESS SMG in Figure 5, which shows that we expect the $B z K$ diagram to classify SMGs at redshifts $z \sim 1-4$ as star-forming $B z K$ s at $z \sim 1.4-2.5$, and SMGs at redshifts greater than $z>4$ as galaxies at $z<1.4$.

We find that the ALESS SMGs display a clear trend with redshift in $S_{8.0} / S_{4.5}$ versus $S_{4.5} / S_{3.6}$ color (Figure 5), with sources at high redshift tending to have higher ratios of 


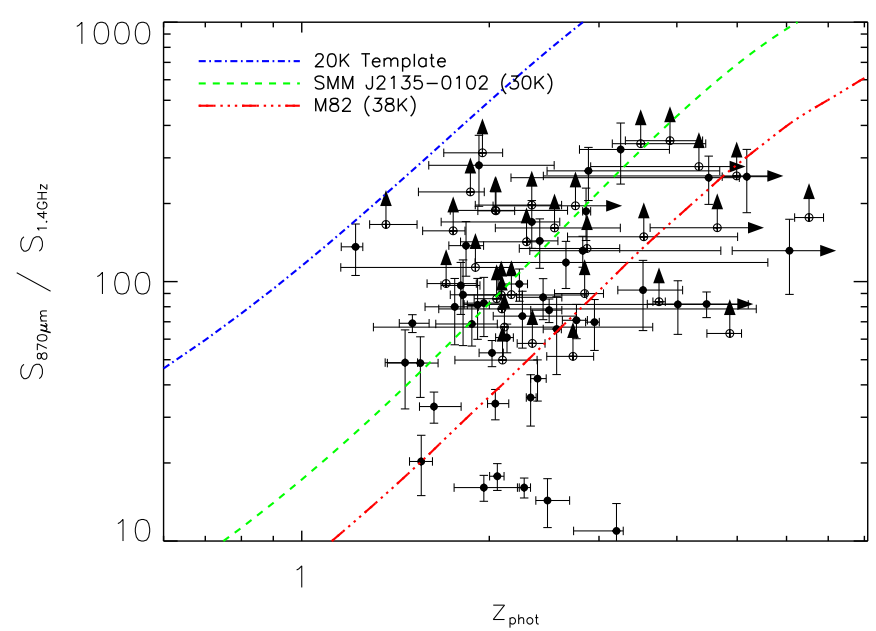

Figure 6. Variation in $S_{870 \mu \mathrm{m}} / S_{1.4 \mathrm{GHz}}$ as a function of redshift for the $77 \mathrm{SMGs}$ with photometric redshifts. We overlay tracks for the local star-forming galaxy M82 $\left(T_{\mathrm{d}}=38 \mathrm{~K}\right)$, SMM J2135-0102 a $z \sim 2.3 \mathrm{SMG}\left(T_{\mathrm{d}} \sim 30 \mathrm{~K}\right)$, and a cool dust template $\left(T_{\mathrm{d}}=20 \mathrm{~K}\right.$; Chary \& Elbaz 2001). The tracks for M82 and SMM J2135-0102 pass through the bulk of the population; however, we find a large dispersion in $S_{870 \mu \mathrm{m}} / S_{1.4 \mathrm{GHz}}$, around $1.5 \mathrm{dex}$ at a fixed redshift. For the 32 SMGs that are not detected at $1.4 \mathrm{GHz}$ we adopt a $3 \sigma$ upper limit (open symbols with arrows), corresponding to $3 \times$ the VLA map rms at the SMG position. The 32 ALESS SMGs that are not detected in available radio data have a range of photometric redshifts from $z_{\text {phot }}>1$. We note that the $S_{870 \mu \mathrm{m}} / S_{1.4 \mathrm{GHz}}$ flux ratios of these undetected, low-redshift $(z \lesssim 2.5)$ SMGs can be adequately reproduced using a "cool" dust template $\left(T_{\mathrm{d}} \stackrel{\sim}{=} 20-30 \mathrm{~K}\right)$, consistent with previous studies (Magnelli et al. 2012; Weiß et al. 2013).

(A color version of this figure is available in the online journal.)

$S_{8.0} / S_{4.5}$. As a further test of our photometric redshifts, we overlay the predicted colors of SMM J2135-0102 (a well-studied SMG at $z=2.3$; Swinbank et al. 2010b) as a function of redshift in Figure 5. We find that the derived photometric redshifts for the ALESS SMGs are in good agreement with the predictions from this SED track.

Ten ALESS SMGs are detected in data taken with the Chandra X-Ray Observatory (see Wang et al. 2013). This $\mathrm{X}$-ray emission is often indicative of an AGN component in the host galaxy, which can affect the SED shape. As such, we now investigate whether the X-ray-detected SMGs (Wang et al. 2013) are distinguishable from the parent sample of SMGs in terms of their IRAC fluxes. We identify one X-ray-detected SMG, ALESS 57.1, which has a high $S_{8.0} / S_{4.5}$ ratio, relative to $S_{4.5} / S_{3.6}$, suggestive of a power-law AGN component in the SED. A further inspection of the SED fits in Appendix A shows that only two SMGs display a clear enhancement in IRAC flux (ALESS 57.1 and 75.1), which is often attributed to AGN-heated dust emission. ${ }^{20}$ The remaining X-ray sources appear well matched to the complete SMG sample. We perform a two-sided Kolmogorov-Smirnov (K-S) test between the X-ray-detected SMGs and the parent sample, in terms of both $S_{8.0} / S_{4.5}$ and $S_{4.5} / S_{3.6}$. The K-S test returns a probability of $85 \%$ that the samples are drawn from the same parent distribution, in terms of both $S_{8.0} / S_{4.5}$ and $S_{4.5} / S_{3.6}$. This suggests that in terms of IRAC color the X-ray-detected SMGs do not represent a distinct subset of SMGs.

20 The low rate of NIR excess in the ALESS SEDs is in stark contrast to the SEDs seen in previous SMG samples, where a large fraction show rest-frame NIR excesses whose amplitude appears to correlate with AGN luminosity (Hainline et al. 2011). This may reflect differences in the sample selection between the predominantly radio-pre-selected, spectroscopically confirmed SMGs in Hainline et al. (2011) and the purely submm-flux-limited sample analyzed here.

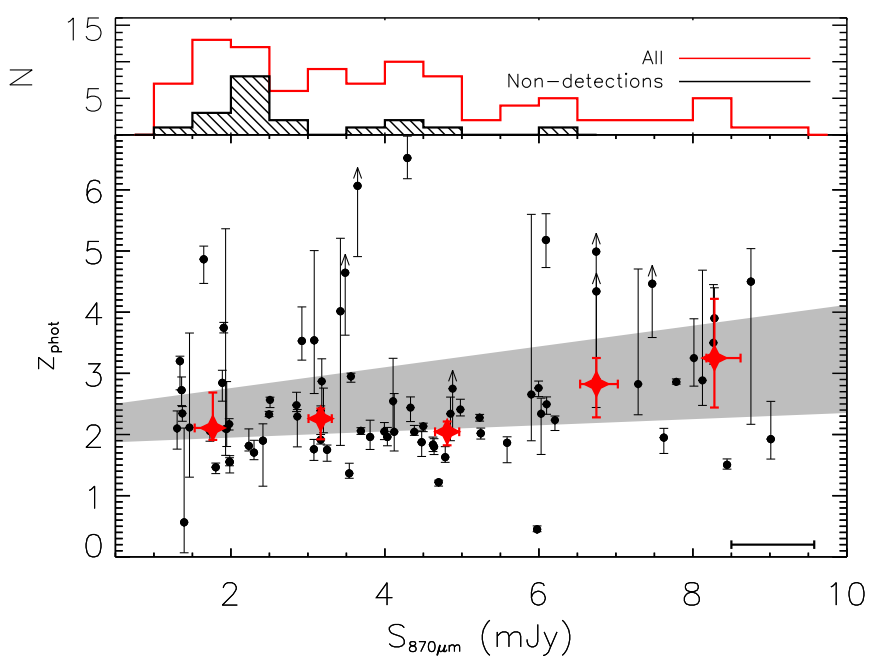

Figure 7. Photometric redshifts of the ALESS SMGs versus their $870 \mu \mathrm{m}$ flux density. We also split the data into $2 \mathrm{mJy}$ bins and plot the median $S_{870 \mu \mathrm{m}}$ and redshift for each bin, with $1 \sigma$ error bars. We test for a trend of higher $S_{870 \mu \mathrm{m}}$ sources lying at higher redshift, but a linear fit to the data shows that the deviation from a constant with redshift is not significant, at $<1.5 \sigma$. A gray region shows the linear fit and associated $1 \sigma$ uncertainty. In the upper panel we highlight the $870 \mu \mathrm{m}$ flux distribution of those SMGs for which we cannot derive photometric redshifts. As we show in Section 3.2.3, these SMGs are likely to lie at $z \geq 3$, and the weak trend we see with redshift is therefore likely driven by incompleteness in our results: including these photometrically faint SMGs would further weaken any trend. The error bar on the median flux density is shown in the lower right.

(A color version of this figure is available in the online journal.)

Finally, we consider the link between $870 \mu \mathrm{m}$ and $1.4 \mathrm{GHz}$ emission, which has been used to identify the optical-NIR counterpart to submm emission. We first stress that we see an order of magnitude of scatter in $S_{870 \mu \mathrm{m}} / S_{1.4 \mathrm{GHz}}$ at a fixed redshift (Figure 6). We now compare the ALESS SMGs to three template SEDs with varying characteristic dust temperatures. We use templates for two well-studied dusty galaxies, SMM J2135-0102 $2^{21}$ ( $\left.30 \mathrm{~K}\right)$ and M82 (38 K). In addition, we also use a $20 \mathrm{~K}$ template drawn from the Chary \& Elbaz (2001) template SED library. These templates span typical dust temperatures for SMGs (Magnelli et al. 2012; Weiß et al. 2013), and we find that they are sufficient to describe the majority of ALESS SMGs. Previous studies have suggested that redshift solutions below $z \lesssim 2.5$ are incorrect for radio-non-detected SMGs (Smolčić et al. 2012). We find that templates with a characteristic temperature of 20-30 K are a plausible explanation for similar ALESS SMGs, and we therefore do not discard redshift solutions at $z \lesssim 2.5$ (see also Swinbank et al. 2014).

\subsubsection{Undetected or Faint Counterparts}

For the 77 ALESS SMGs that have counterparts in at least four optical or NIR bands, we are able to estimate reliable photometric redshifts. However, this leaves 19 ALESS SMGs ( $\sim 20 \%$ of the sample) that do not have sufficient detections to derive a photometric redshift. These 19 SMGs have a median detection significance of $4.0 \pm 0.3 \sigma$, at $870 \mu \mathrm{m}$ (see Figure 7), and so we initially test whether they are spurious sources or simply fainter at optical-near-infrared wavelengths than the rest of the population. First, we divide the SMGs detected in fewer

\footnotetext{
21 The best-fit FIR SED to the photometry of SMM J2135-0102 is a two-component dust model at $30 \mathrm{~K}$ and $60 \mathrm{~K}$. The dust masses of each component are $M_{\mathrm{d}}^{\mathrm{warm}}=10^{6} M_{\odot}$ and $M_{\mathrm{d}}^{\text {cold }}=4 \times 10^{8} M_{\odot}$ (Ivison et al. 2010).
} 

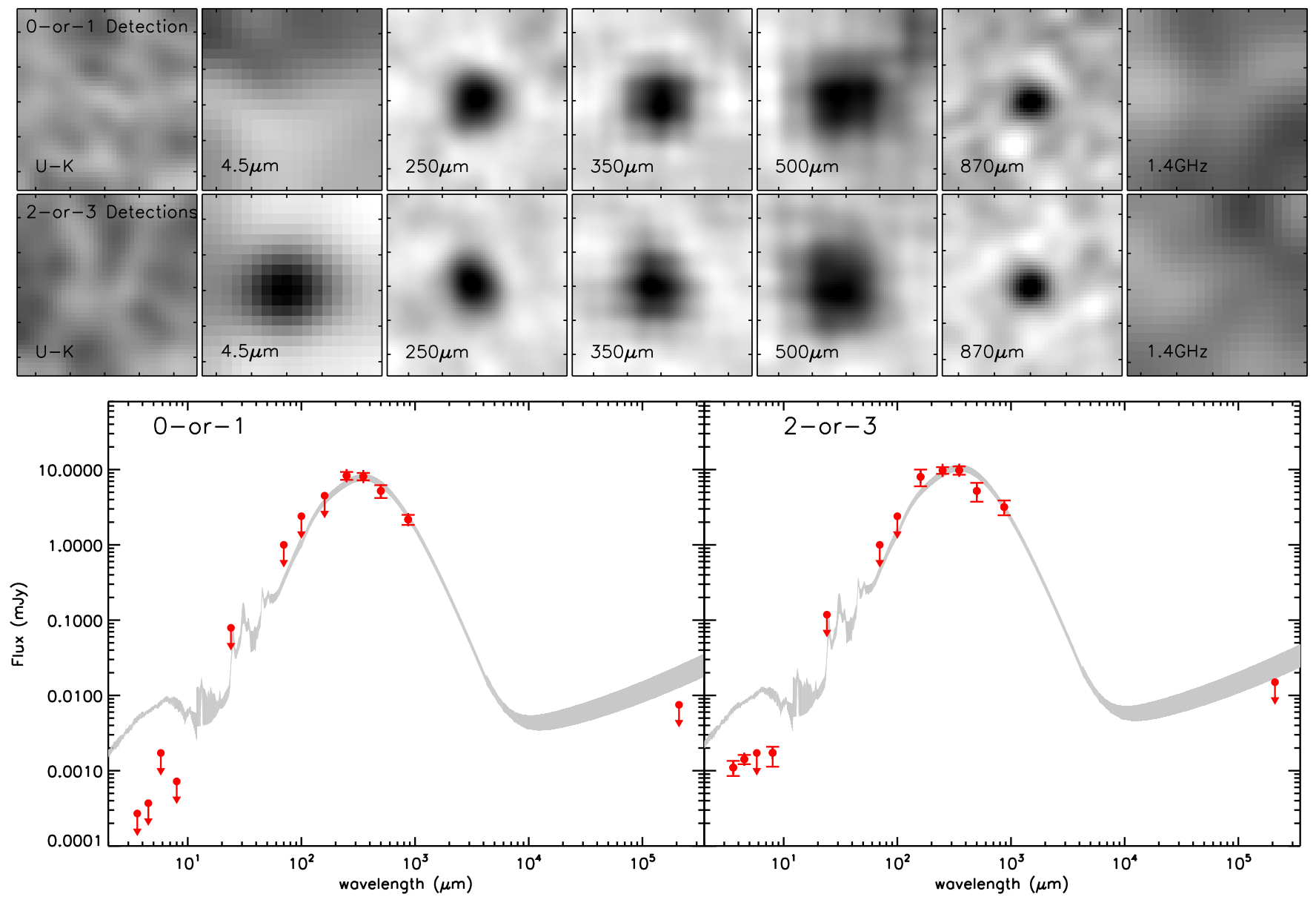

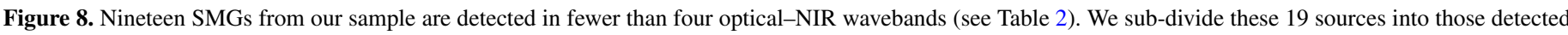

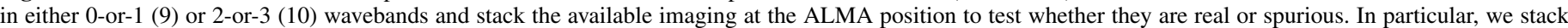

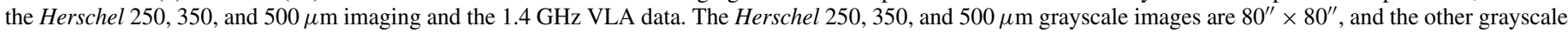

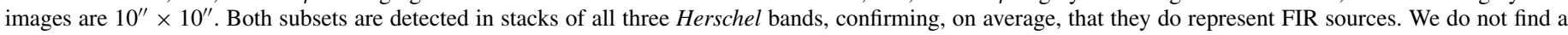

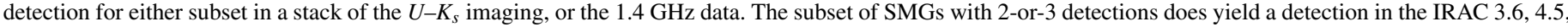

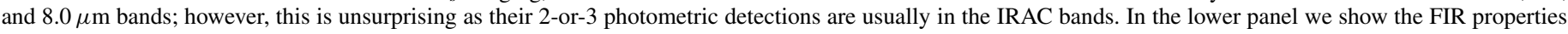

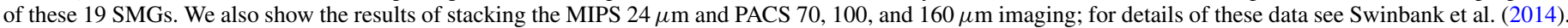

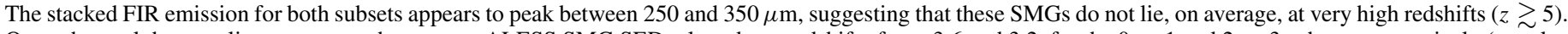

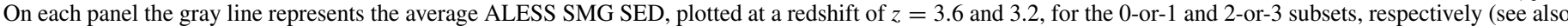

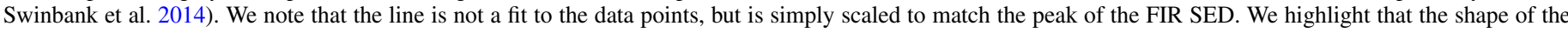

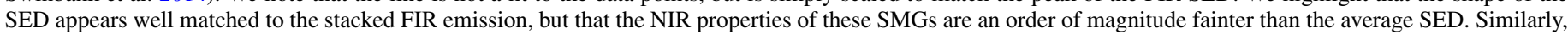
at the nominal redshift plotted the composite SED overpredicts the radio emission from both subsets of SMGs.

(A color version of this figure is available in the online journal.)

than 4 wave-bands into subsets comprising 0-or-1 and 2-or-3 detections in both the optical $\left(U-K_{S}\right)$ and IRAC wavebands and first stack their emission in these wavebands using a clipped mean algorithm. Figure 8 shows that only the 2 -or-3 waveband subset yields a stacked detection in the IRAC wavebands at the $7 \sigma$ level, whereas the optical stacks of both subsets and the IRAC stack of the 0-or-1 subset all yield non-detections at the $<3 \sigma$ level.

Next, we stack the emission from these SMGs in the FIR Herschel/SPIRE maps at 250, 350, and $500 \mu \mathrm{m}$ and show these in Figure 8. The SMGs are clearly detected at $>4 \sigma$ in all SPIRE bands in both the 0-or-1 and 2-or-3 subsets, with 250, 350, and $500 \mu \mathrm{m}$ flux densities between 4 and $16 \mathrm{mJy}^{22}$ We note that four of the SMGs are detected individually at $250 \mu \mathrm{m}$, two of which

\footnotetext{
22 We use the deblended SPIRE maps described in Swinbank et al. (2014), but to account for the clustering, we use a deblended map where the ALESS SMGs are not included in the a priori catalog.
}

are detected at 350 and $500 \mu \mathrm{m}$. The SEDs for these stacks peak between 250 and $350 \mu \mathrm{m}$ for both the 0 -or- 1 and 2-or-3 subsets, as shown in Figure 8. In this figure we also overlay the composite ALESS SMG SED (see Section 3.3), redshifted to $z=3.2$ and $z=3.6$ for the 0 -or- 1 and 2 -or- 3 subsets, respectively, to match the peak of the FIR SED. The redshifted template appears to roughly reproduce the FIR properties of these SMGs, although we note that their NIR properties are approximately an order of magnitude fainter than the composite ALESS SMG SED. We caution that variation in the dust temperature or redshift of the SMGs in the 0-or-1 and 2-or-3 subsets would smear the peak wavelength of the stacked FIR SED. Thus, the FIR SED of the 0 -or-1 and 2-or-3 subsets peaking at longer wavelengths is only tentative evidence that they lie at higher redshift. A full discussion of the FIR properties of these SMGs is presented in Swinbank et al. (2014).

In Figure 9 we plot the $H$-band absolute magnitude $\left(M_{H}\right)$ versus redshift for the 77 ALESS SMGs where we have derived 


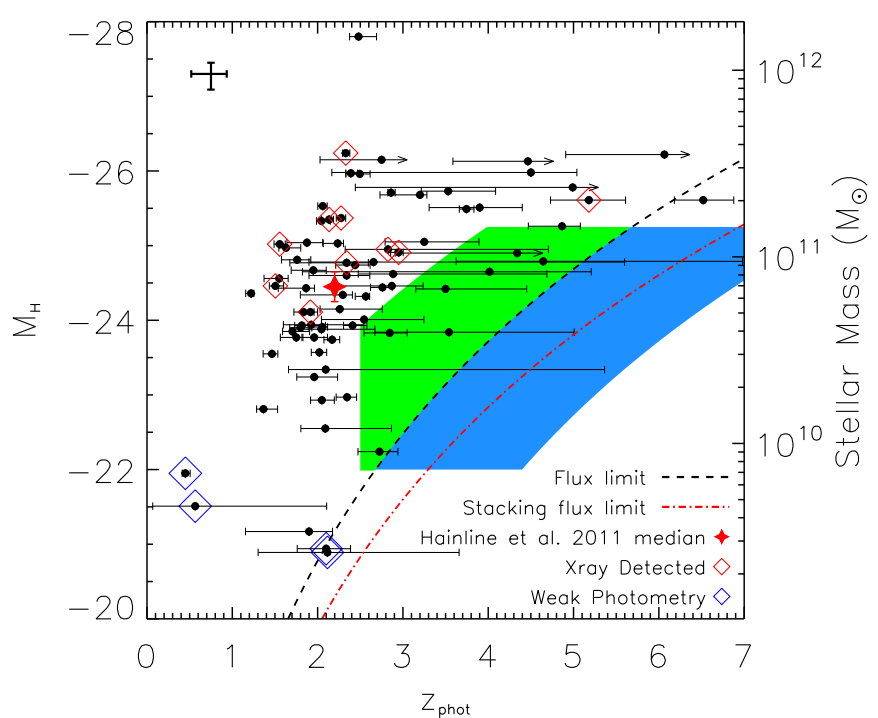

Figure 9. Absolute $H$-band magnitude distribution, derived from SED fits to the observed photometry. The median $M_{H}$ of the ALESS SMGs is $-24.56 \pm 0.15$, which agrees with that derived for the SMG sample presented by Hainline et al. (2011), $M_{H}=-24.45 \pm 0.20$. The dashed line illustrates the flux-limited nature of our survey, and the red line the limit in our IRAC stacking (flux limit taken from the IRAC $4.5 \mu \mathrm{m}$ limiting magnitude). By requiring that the $M_{H}$ distribution is not bimodal, and using the IRAC selection limits, we can estimate the redshift distribution for those ALESS SMGs without sufficient photometry to derive photometric redshifts. Shaded regions represent the area populated by ALESS sources detected in 0-or-1 (blue) or 2-or-3 (green) wavebands. For each source we determine a mass-to-light ratio, $M / L_{H}$, from the $\mathrm{SFH}$ returned in the SED fitting and estimate a median stellar mass for the complete sample of 96 SMGs of $M_{\star}=(8 \pm 1) \times 10^{10} M_{\odot}$, for a Salpeter IMF. We caution that due to the unknown SFHs, the stellar masses of the ALESS SMGs are very poorly constrained (see Section 4.3). Sources that are possible gravitational lenses, or with questionable photometry, are highlighted with blue diamonds.

(A color version of this figure is available in the online journal.)

a photometric redshift. We also highlight the survey selection limits, which show that between $z=0$ and $z \sim 2.5$ the NIR survey limits should be complete at magnitudes brighter than $M_{H}=-22$ (equivalent to a stellar mass of $M_{\star} \sim 10^{10} M_{\odot}$ for a light-to-mass ratio of $L_{H} / M_{\star} \sim 3.8$; Hainline et al. 2011). However, above $z \sim 2.5$, the optical-NIR survey limits mean that only the brightest SMGs are detected, despite the $870 \mu \mathrm{m}$ selection ensuring that we have an unbiased sample of SMGs from $0<z \lesssim 6$. We make the assumption that the absolute $H$-band magnitude distribution of the ALESS SMGs is complete at $z<2.5$ and that incompleteness in the distribution at $z>2.5$ is due to our NIR selection limits, i.e., that the 19 SMGs detected in $<4$ wavebands lie at $z>2.5$ and that the absolute $H$-band magnitude distribution is not bimodal. Our assumption is in agreement with Figure 8, which shows that the stacked FIR SED of these SMGs peaks at longer wavelengths than the average ALESS SMGs, and indeed one of the SMGs detected in $<4$ wavebands, ALESS 65.1, has been spectroscopically confirmed to be at $z=4.4$ (Swinbank et al. 2012). We caution that an alternative explanation is that the SMGs detected in 0-or-1 and 2-or-3 wavebands are either significantly more dust obscured $\left(A_{V}>4\right)$ or lower stellar mass $\left(M_{\star}<10^{10} M_{\odot}\right)$ than the optical-NIR detected ALESS SMGs, but note that this would mean that both properties have a bimodal distribution.

To estimate the likely redshift distribution of the 19 ALESS SMGs that are detected in $<4$ bands, we first assume that the survey is complete in $M_{H}$ at $z<2.5$ (Figure 9). To determine incompleteness in the magnitude distribution at $z>2.5$, we construct $\sim 1000$ realizations of the ALESS SMG $H$-band absolute magnitude distribution over the range $z=0-2.5$ and compare this to the $H$-band absolute magnitude at $z>2.5$. We then assign values of $M_{H}$ to the 10 ALESS SMGs detected in 2-or-3 wavebands to minimize the incompleteness in the $H$-band absolute magnitude above $z>2.5$. These 10 sources have a stacked flux close to our photometric selection limit and hence are assigned redshifts based on the selection limit at the corresponding value of their $M_{H}$. We repeat this procedure for the remaining nine ALESS SMGs detected in the 0-or-1 wavebands. Since these SMGs are not detected in our optical or NIR stacking, we assume that these SMGs must lie below (or close to) the detection limit in our stacked IRAC maps. We caution that, in both cases, this may underestimate the redshift of these SMGs, although since both subsets peak at $\sim 350 \mu \mathrm{m}$ in the Herschel stacks in Figure 8, it appears that on average they do not lie at very high redshifts $(z \gtrsim 5)$. Using this approach, the median redshift of the ALESS SMGs is $z \sim 3.5$ and $z \sim 4.5$ for sources detected in 2-or-3 and 0-or-1 wavebands, respectively, similar to the redshifts derived from the composite SMG SED (Figure 8). Including these redshifts in our redshift distribution, the median photometric redshift for our complete sample of 96 ALESS SMGs is then $z_{\text {phot }}=2.5 \pm 0.2$ (Figure 12). The distribution has a tail to high redshift, and $35 \% \pm 5 \%$ of the ALESS SMGs lie at $z_{\text {phot }}>3$.

\subsubsection{ALMA Blank Maps}

We have now discussed the redshift distribution of all SMGs in the ALESS MAIN catalog. Before continuing, it is important to consider the ALMA maps in which we do not detect any SMGs. In total we obtained high-quality ALMA observations of 88 LABOCA submm sources. Of these 88 ALMA observations, 19 are blank maps and do not contain an SMG above $\mathrm{S} / \mathrm{N}>3.5$ within the primary beam (Hodge et al. 2013). The ALMA blank maps are predominantly faint LABOCA detections and compose 14 out of 24 LABOCA detections with $S_{870}<5.5 \mathrm{mJy}$. To verify the reliability of the original LABOCA detections, we stack the FIR emission from all 19 sources in the FIR Herschel/ SPIRE maps at 250, 350, and $500 \mu \mathrm{m}$. We detect emission at $>8 \sigma$ in our stacks of all three SPIRE wavebands and show the images of each stack in Figure 10. Furthermore, we split the sample at a detection significance of $4.2 \sigma$ in the original LABOCA map, yielding subsets containing 10 and 9 sources, respectively. We again stack the FIR emission for both subsets and detect emission at $>6 \sigma$ at 250,350 , and $500 \mu \mathrm{m}$ in both subsets, again confirming that on average both subsets contain real sources. The results of our stacking analysis are consistent with Weiß et al. (2009), who state that only $\sim 3$ of the 88 LESS submm sources are expected to be false detections. ${ }^{23}$

Our ALMA observations have demonstrated that single-dishdetected submm sources often fragment into multiple SMGs in interferometric observations (Karim et al. 2013; Hodge et al. 2013; see also Barger et al. 2012). We now test whether it is possible that the ALMA blank maps similarly contain multiple SMGs, each below the $870 \mu \mathrm{m}$ flux limit of the ALESS survey, but which together appear as a single, blended source in the

\footnotetext{
23 Weiß et al. (2009) predict that the complete LESS sample of 126 submm sources contains five false detections. In addition, Weiß et al. (2009) consider the effects of map noise on measured source fluxes, which boosts otherwise faint sources above the nominal flux limit of their catalog. However, they do not account for source clustering in their analysis, which may result in a higher flux boost.
} 

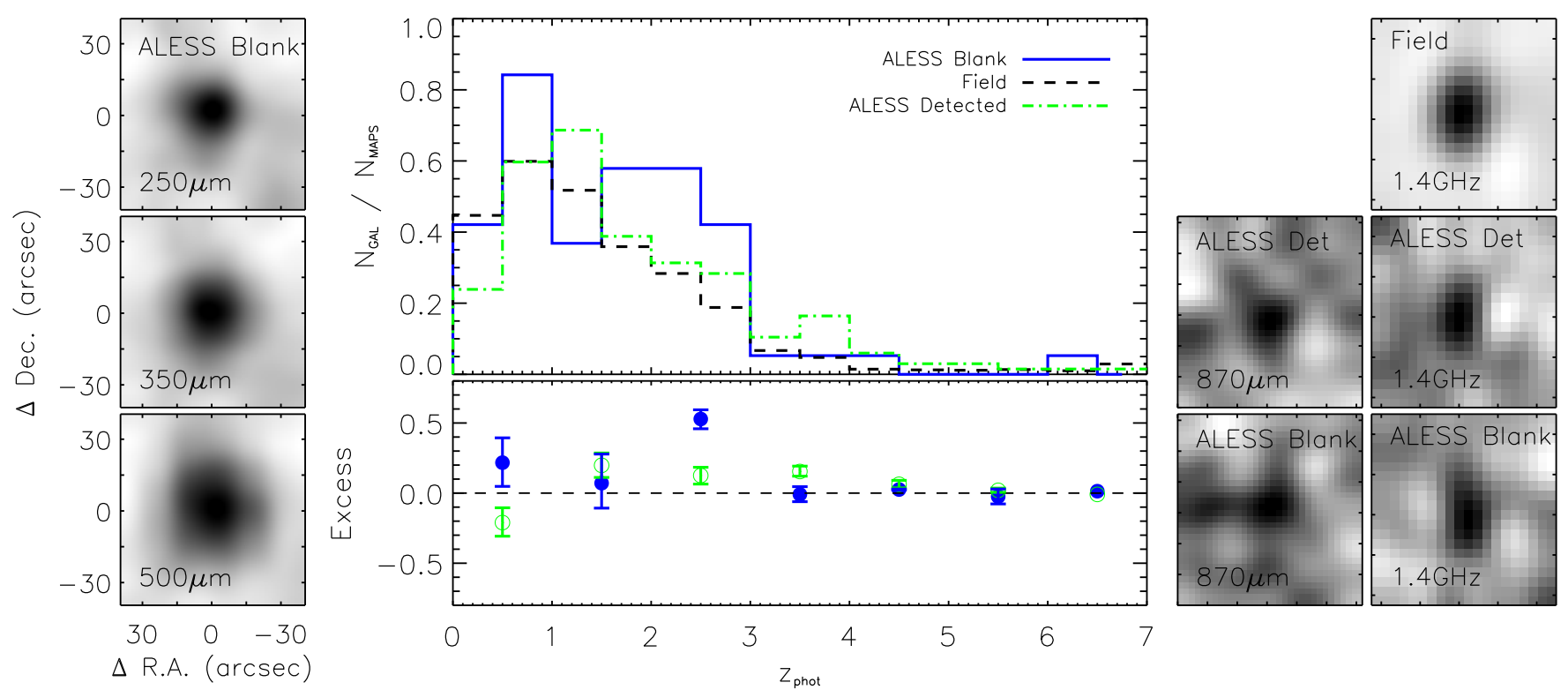

Figure 10. Left: 19 of the 86 ALMA maps of LESS submm sources considered in this work do not contain an SMG brighter than our $870 \mu \mathrm{m}$ detection threshold of $\sim 1.4$ mJy, within the ALMA primary beam. We show the results of stacking the Herschel/SPIRE 250, 350, and $500 \mu \mathrm{m}$ maps at the position of these 19 LABOCA detections. We detect emission at $>8 \sigma$ at all three SPIRE wavelengths, confirming that at least on average these sources are real. Middle: We compare the redshift distribution of IRAC sources in ALMA maps without SMGs to the field and to those in areas covered by ALMA maps containing detected SMGs. The field sample is drawn from random apertures with the same size as the ALMA primary beam, and any ALESS SMGs are removed from all samples. In the maps with detections we find that the redshift distribution is consistent with the field, but in the blank ALMA maps we find an excess of sources at $z \sim 2.5$. This suggests that the LESS SMGs have fragmented into multiple components below our detection threshold, and that they have a redshift distribution consistent with the $S_{870} \mu \mathrm{m}$-brighter ALESS sample. Right: Stacked maps of $3.6 \mu \mathrm{m}$ selected galaxies with photometric redshifts between $z=1$ and 3 . We stack the $870 \mu \mathrm{m}$ emission for sources covered by our ALMA observations and the $1.4 \mathrm{GHz}$ emission for all galaxies. We again split the sample into subsets based on whether they are in an ALMA map or not, and furthermore into ALMA blank maps and ALMA maps containing SMGs. We obtain a $\sim 4 \sigma$ detection of the IRAC samples in both $870 \mu \mathrm{m}$ stacks. The ALMA maps lacking SMGs have a primary-beam-corrected flux of $S_{870}=0.36 \pm 0.09 \mathrm{mJy}$ and a number density of sources $\sim 2 \times$ higher than the field over the range $z=1-3$. We detect all subsets at $1.4 \mathrm{GHz}$, and we find that the IRAC sources in the ALMA maps are $\sim 2.5 \times$ brighter at $1.4 \mathrm{GHz}$ than those in the general field population at $2.8 \sigma$. This tentative result suggests that IRAC-selected sources are typically brighter at $870 \mu \mathrm{m}$ when in the vicinity of a submm source.

(A color version of this figure is available in the online journal.)

LABOCA observations. First, we use the photometric redshifts derived for the $3.6 \mu \mathrm{m}$ training set to search for an excess of $3.6 \mu \mathrm{m}$ sources in the ALMA blank maps, when compared to the field (see Figure 10). We construct the redshift distribution for the field by placing 1000 random apertures of equal size to the ALMA primary beam across the ECDFS. We compare the redshift distribution in these random fields to that of sources in the ALMA blank maps and identify an excess of $0.61 \pm 0.07$ sources per ALMA blank map across the redshift range $z=2-3$. There is also a small excess of $0.15 \pm 0.06$ of $3.6 \mu \mathrm{m}$ selected sources in the ALMA maps containing an SMG, over the same redshift range $z=2-3$, compared to the field, where we have removed the ALESS SMG counterparts from the comparison. The existence of a small excess suggests that the ALMA blank maps contain multiple faint SMGs, and crucially that they have a redshift distribution broadly similar to the ALESS SMGs.

We assess the $870 \mu \mathrm{m}$ flux contribution of these IRAC sources by stacking the primary-beam-corrected ALMA maps at the position of the $3.6 \mu \mathrm{m}$ sources, again removing all ALESS SMGs in the MAIN catalog from the sample. In Figure 10 we show $870 \mu \mathrm{m}$ stacks for $3.6 \mu \mathrm{m}$ sources, over the redshift range $z=1-3$, in both ALMA blank maps ("Blank") and ALMA maps containing at least one SMG ("Detected"). We choose to stack over the redshift range $z=1-3$ as it covers the observed excess in IRAC sources and the expected range of redshifts for the bulk of the SMGs. Both "Blank" and "Detected" subsets are detected at a significance of $\sim 4 \sigma$ in the $870 \mu \mathrm{m}$ stacks, and the ALMA blank maps have an average primary-beam-corrected peak flux of $S_{870}=0.36 \pm 0.09 \mathrm{mJy}$ (the sources in maps with detected SMGs yield $S_{870}=0.29 \pm 0.08 \mathrm{mJy}$ ). Using the number density of the $3.6 \mu \mathrm{m}$ sources, we calculate that these contribute a total $870 \mu \mathrm{m}$ flux per ALMA blank map of $S_{870}=0.76 \pm 0.19 \mathrm{mJy}$.

However, we also need to confirm that these $3.6 \mu \mathrm{m}$ sources in the ALMA maps are brighter in the submm than the IRAC population outside the ALMA fields. This is difficult as we only have ALMA coverage of the LABOCA source positions, but we can take advantage of the radio coverage of the whole field to use this as a proxy to estimate the relative brightness of these two samples. We therefore stack the $1.4 \mathrm{GHz}$ VLA map at the positions of the $3.6 \mu \mathrm{m}$ sources, at $z=1-3$, in the ALMA blank maps and the surrounding field. We note that we do not account for resolved radio emission in our stacking, but at $z=2$ the resolution of the $1.4 \mathrm{GHz}$ map is $\sim 25 \times 14 \mathrm{kpc}$ and we do not expect SMGs to be significantly resolved on these scales (Biggs \& Ivison 2008). We measure $\sim 2.5 \times$ higher radio fluxes, at a significance of $2.8 \sigma$, for $3.6 \mu \mathrm{m}$ sources at $z=1-3$ in the ALMA "Blank" and "Detected" maps compared to the field. We note that this analysis is limited by the small number of $3.6 \mu \mathrm{m}$ sources considered in the ALMA maps and the depth of the radio map combined with the expected faint $1.4 \mathrm{GHz}$ flux distribution of SMGs (Figure 2). If we instead only consider the ALMA "Blank" maps, we measure $\sim 3.5 \times$ higher radio fluxes, at a decreased significance of $2.0 \sigma$, compared to the field. The significance of these results means that they only provide tentative evidence that the ALMA maps contain $3.6 \mu \mathrm{m}$ 
sources, at $z=1-3$, which are typically brighter in the submm, compared to the field.

The flux limit of the original LESS survey was $4.4 \mathrm{mJy} \mathrm{beam}^{-1}$, and hence our results are insufficient to fully explain the ALMA blank maps. There are two important caveats with this result. The first is that we expect at least three of the LABOCA sources to be spurious detections, which will downweight our stacking results to lower values of $S_{870}$. The second is that although we selected sources at $3.6 \mu \mathrm{m}$, the requirement for a photometric redshift means that each source must be detected in $>4$ wavebands. If we have the same proportion of sources detected in $<4$ wavebands as the MAIN sample, i.e., 20\%, this would explain a further fraction of the missing flux. Although we cannot explain all of the missing flux, these results do indicate that the ALMA blank maps contain multiple faint SMGs, below the detection limit of the ALESS survey. Crucially, we find that there is an excess of sources at $z \sim 2.5$ in these maps, which suggests that the redshift distribution of faint SMGs appears to match the ALMA-detected SMGs.

\subsection{Constraints on $\mathrm{SFH}$}

The primary use of HYPERZ is to derive photometric redshifts; however, in the SED fitting procedure HYPERZ also determines the best-fit SFH for each source. We now investigate the reliability of the returned SFH parameters. We find that 52 $(68 \%), 15(19 \%), 6(8 \%)$, and $4(5 \%)$ of the ALESS SMGs have SFHs corresponding to the burst, $1 \mathrm{Gyr}, 5 \mathrm{Gyr}$, and constant templates, respectively. While this appears to indicate a strong preference for the instantaneous burst $\mathrm{SFH}$, we test for degeneracy in our results by re-running HYPERZ allowing just the constant or just the burst SFHs. The SED fits, for the two SFHs, are indistinguishable, with a median $\Delta \chi_{\text {red }}^{2}$ between the constant and burst SFH of $0.34_{-0.09}^{+0.16}$. The SED fits return a median age of $35 \pm 15 \mathrm{Myr}$ and $1.0 \pm 0.4 \mathrm{Gyr}$ for the burst and constant SFHs, respectively. In Section 4.3 we discuss the uncertainties introduced into stellar mass estimates for SMGs from these unconstrained SFHs.

To investigate whether we can extract any further information about the SFHs from the ALESS photometry, we construct the SED for the "average" ALESS SMG. In Figure 11, we present the de-redshifted photometry for the ALESS SMGs normalized by rest-frame $H$-band luminosity. The composite SED shows a steep red spectrum consistent with strong dust reddening, as expected for SMGs. However, there may also be a hint of a break at $\sim 4000 \AA$. If this feature is indeed real, it is most likely from a Balmer break, which would suggest the presence of stars with ages $\geqslant 10^{8}$ yr. We derive photometry for the average ALESS SMG by convolving the running median with the photometric filters used in this work. As we observe a hint of a Balmer break in the SED of the typical SMG, which could help differentiate between the SFHs, we also include an extra filter close to the break to provide a stronger test of the similarity of the models to the average photometry in this area (for this we use a $Y$-band filter shifted in wavelength to lie directly between the $z$ and $J$ filters). We then fit the average photometry redshifted to $z=2.5$ using HYPERZ and compare to both the constant and instantaneous burst SFHs.

The constant SFH provides the best fit to the median SED $\left(\chi_{r}=1.0\right)$; however, we cannot reliably distinguish the models, which have $\Delta \chi_{\mathrm{r}}=0.2$. The constant SFH has a burst age of $2.3 \mathrm{Gyr}, A_{V}=1.5$ and corresponding $L_{H} / M_{\star}$ of $\sim 3$, while the instantaneous burst has an age of $30 \mathrm{Myr}, A_{V}=1.8$, and

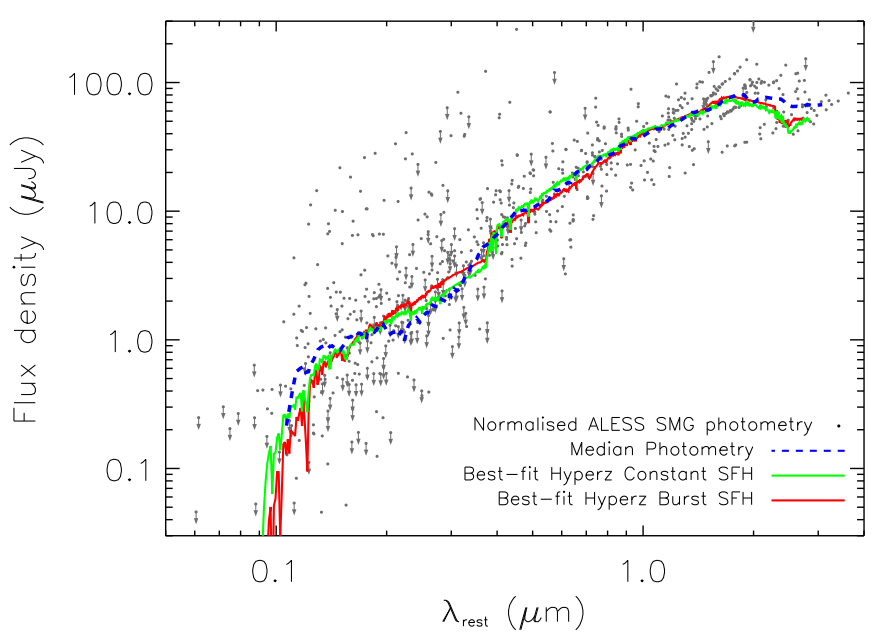

Figure 11. Photometry for the ALESS SMGs, de-redshifted and normalized by their median $H$-band absolute magnitude. We show the running median (dashed line), which represents the SED of an average SMG and exhibits a steep red spectrum indicative of strong reddening but with a hint of a break at $0.4 \mu \mathrm{m}$ due to Balmer or $4000 \AA$ break. We indicate non-detections with arrows and set the flux for these values to zero when calculating the running median. To test whether we can distinguish between different SFHs for the ALESS SMGs, we measure the median photometry through each filter in Table 1. We perform SED fitting on the average photometry using HYPERZ, but allow only the two extremes of SFH, a constant SFH and an instantaneous burst. The best fit corresponds to a constant SFH with an age of $2.3 \mathrm{Gyr}$ and $A_{\mathrm{v}}=1.5$ (best-fit Burst; $30 \mathrm{Myr}$ and $A_{\mathrm{v}}=1.8$ ); however, we find that the two SFHs are indistinguishable with $\Delta \chi^{2}=0.2$. We use the Constant SFH SED to create an average optical-FIR SMG template, which is presented in Swinbank et al. (2014).

(A color version of this figure is available in the online journal.)

corresponding $L_{H} / M_{\star}$ of $\sim 15$. The derived $L_{H} / M_{\star}$ and ages are very different, and we conclude that even for the limited selection of SFHs we consider for the ALESS SMGs it is not possible to distinguish between each SFH in a statistically robust manner. This is in agreement with previous work that demonstrates the difficulty in constraining the individual SFHs of high-redshift SMGs with SED fitting (Hainline et al. 2011; Michałowski et al. 2012).

Although we find that it is not possible to distinguish between the SFHs of the ALESS SMGs, the reddening correction returned by HYPERZ appears consistent. Considering all SFHs, we find a median reddening correction of $A_{V}^{\text {all }}=1.7 \pm 0.1$, and $A_{V}^{\text {const }}=2.0 \pm 0.1$ for the constant SFH alone. The reddening correction is an average correction across the entire galaxy; however, the dust in SMGs is likely to be clumpy (Swinbank et al. 2010b; Danielson et al. 2011; Hodge et al. 2012; Menéndez-Delmestre et al. 2013), and as such it is likely to be considerably higher in the star-forming regions. To confirm this, we derive SFRs from the dust-corrected restframe UV emission, at $1500 \AA$, of each ALESS SMG, following Kennicutt (1998), and compare these values to the FIR SFRs derived by Swinbank et al. (2014). To bring the UV-derived SFR into agreement with $\mathrm{SFR}_{\mathrm{FIR}}$ requires a median reddening correction of $A_{V}=2.4 \pm 0.1$, or an additional $\sim 0.7 \mathrm{mag}$ to the $A_{V}$ derived from SED fitting, indicating that star formation in the SMGs is occurring in highly obscured regions. We note that the UV-derived SFR indicator is only likely to be reliable for a constant SFR SFH at ages of $>100 \mathrm{Myr}$, and as such it is likely that our UV-derived SFR is overestimated, and that the reddening correction is higher than $A_{V} \sim 2.4$. 


\section{DISCUSSION}

\subsection{Redshift Distribution}

The complete redshift distribution of the 96 ALESS SMGs in our sample has a median redshift of $z_{\text {phot }}=2.5 \pm 0.2$ and a tail to high redshift, with $35 \% \pm 5 \%$ of sources lying at $z>3$. As an initial comparison to the ALESS SMGs, we use the spectroscopically confirmed, radio-identified, SMG sample from Chapman et al. (2005; C05). The C05 sample has a median redshift of $z=2.2 \pm 0.1$, in agreement with our results; however, there are notable discrepancies between the samples. The C05 sources are radio-selected, which, due to the positive $K$-correction, is likely to bias their results to lower redshifts. Indeed, the highest redshift SMG in the C05 sample is $z=3.6$, and the distribution does not show such a pronounced tail to high redshifts as we observe in the ALESS SMG distribution. We also note differences between the samples at $z<1.5$. The C05 sample contains a significant number of SMGs at these redshifts (25\%), but only five ALESS SMGs, or 6\%, lie at $z<1.5$. A two-sided K-S test between the ALESS SMGs and C05 indicates that there is a $13 \%$ probability that the samples are drawn from the same parent distribution. A fairer comparison is to consider only the ALESS SMGs with radio fluxes $S_{1.4}>40 \mu \mathrm{Jy}$, roughly the selection limit of the C05 sample. Here, the median redshift of $S_{1.4}>40 \mu \mathrm{Jy}$ ALESS SMGs is $z_{\text {phot }}=2.3 \pm 0.1$, and the above analysis remains unchanged. We note that the median redshift of the radio-detected ALESS SMGs is $z_{\text {phot }}=2.3 \pm 0.1$ and is lower than the radio non-detections, which have a median redshift of $z_{\text {phot }}=3.0 \pm 0.3$.

We caution that the ALESS sample is selected from the original LABOCA survey, which had a detection threshold of $4.4 \mathrm{mJy}$. Our ALMA observations reach a typical depth of $1.4 \mathrm{mJy}(3.5 \sigma)$, and so we have SMGs in our sample below the original LABOCA limit. These SMGs are biased in their selection and are only in our SMG sample due to their onsky clustering with other SMGs. It is difficult to quantify the effect of these SMGs on our redshift distribution, but we note that we do not see any significant trend between redshift and $870 \mu \mathrm{m}$ flux density (see Figure 7). If we split the ALESS SMGs into subsamples based on the LABOCA detection limit, we find that the median redshift for SMGs above $4.4 \mathrm{mJy}$ is $z_{\text {phot }}=2.5 \pm 0.2$, and $z_{\text {phot }}=2.6 \pm 0.3$ below $4.4 \mathrm{mJy}$. However, we should also consider the ALMA maps where the original LABOCA source has not fragmented into multiple components. The median redshift of these 45 "isolated" SMGs is $z_{\text {phot }}=2.3 \pm 0.2$, consistent with the complete sample of 96 SMGs.

A number of SMGs in our sample have secondary redshift solutions (which correspond to secondary minima in $\chi^{2}$, e.g., Figure 14) or have large uncertainties in their photometric redshifts. To investigate whether these could significantly affect the shape of the redshift distribution, we calculate the redshift probability distribution for each SMG and normalize the integral of the distribution. For the SMGs detected in $<3$ wavebands we assign a uniform probability distribution between the detection limits described in Section 3.2.3. We combine the redshift probability distributions for each SMG and show the combined redshift distribution in Figure 12. We find that the redshift distribution derived from the combined probability distributions is in excellent agreement with the "best-fit" redshift distribution, indicating that while secondary minima and large redshift uncertainties are important for individual sources, they do not significantly affect the shape of the redshift distribution.
In Figure 12 we show the redshift distribution of the ALESS SMGs as a function of look-back time. The distribution is well described by a Gaussian $\left(\chi_{r}^{2}=0.99\right)$ of the form

$$
N(T)=A e\left[-\left(T-T_{0}\right)^{2} / 2 \sigma_{\mathrm{T}}^{2}\right],
$$

where $A=14.10 \pm 0.55, T_{0}=11.10 \pm 0.05$, and $\sigma_{\mathrm{T}}=$ $1.07 \pm 0.05$ (of course, this function extends beyond the Hubble time and hence must be truncated at $13.7 \mathrm{Gyr}$ ). We note that the high-redshift tail to the distribution is a less pronounced feature when the distribution is parameterized, linearly, by age. ${ }^{24}$

One of the main results from our ALESS survey is that the "robust" Radio/1.4 GHz and MIPS/24 $\mu \mathrm{m}$ identifications for the multiwavelength counterpart to the original LABOCA detection were only $80 \%$ correct and $45 \%$ complete (Hodge et al. 2012). As such, we do not compare our results to redshift distributions derived from single-dish submm/mm surveys (i.e., Aretxaga et al. 2007; Chapin et al. 2009; Wardlow et al. 2011; Yun et al. 2012; Casey et al. 2013). Instead, we restrict the comparison to recent millimeter interferometric observations of other, albeit small, samples of SMGs. First, we compare to the 28 SMGs from Smolčić et al. (2012), which can be split into two distinct subsets: (1) $171.1 \mathrm{~mm}$ selected sources, with follow-up observations at $890 \mu \mathrm{m}$ with the Submillimeter Array (SMA); and (2) $16870 \mu \mathrm{m}$ selected sources, with follow-up observations at $1.3 \mathrm{~mm}$ with the Plateau de Bure Interferometer. Five sources are duplicated in both samples.

The $1.1 \mathrm{~mm}$ selected sample from Smolčić et al. (2012) has a median redshift of $z=2.8 \pm 0.4$, which is composed of a mixture of seven spectroscopic redshifts, seven photometric redshifts, and three redshifts derived from the mm-radio relation (see Figure 6). Due to the shape of the FIR SED, we might expect samples selected at longer wavelength to lie at higher redshift, and indeed we observe this for the ALESS SMGs when the sample is split into detections that peak at 250, 350, and $500 \mu \mathrm{m}$ (Swinbank et al. 2014). As such it is unsurprising that the $1.1 \mathrm{~mm}$ selected sample from Smolčić et al. (2012) has a marginally higher median redshift, although we note that within the errors it is in agreement with the median of the ALESS SMGs. The second sample consists of $870 \mu \mathrm{m}$ selected galaxies, with interferometric observations at $1.3 \mathrm{~mm}$. The initial selection criterion at $870 \mu \mathrm{m}$ means that the sample is a closer match to the ALESS SMGs (although they must still be brighter than $\sim 1.5 \mathrm{mJy}$ at $1.3 \mathrm{~mm}$ ), and indeed the median redshift is $z=2.6 \pm 0.6$ (five spectroscopic/eight photometric/three mm-radio redshifts), in good agreement with the results presented here.

Overall the combined mm and submm samples from Smolčić et al. (2012) contain 28 SMGs with a median redshift of $z=2.6 \pm 0.4$, in agreement with the ALESS SMGs. We note that redshifts for five SMGs from the Smolčić et al. (2012) sample are derived from the mm-radio relation and are claimed to lie at $z>2.6$. As we have noted, this relation displays an orderof-magnitude scatter at a fixed redshift (Figure 6); however, these sources are not detected in the photometry employed by

\footnotetext{
${ }^{24}$ We note that the ALESS SMG redshift distribution is well described by a lognormal distribution of the form$$
\frac{d N}{d z}=\frac{B}{(z-1) \sigma_{\mathrm{z}}} e^{-\left[(\ln (z-1)-\mu)^{2} / 2 \sigma_{\mathrm{Z}}^{2}\right]},
$$

where $B=89.2 \pm 1.7, \mu=1.53 \pm 0.02$ and $\sigma_{\mathrm{z}}=0.59 \pm 0.01$ (see also Yun
} et al. 2012). 

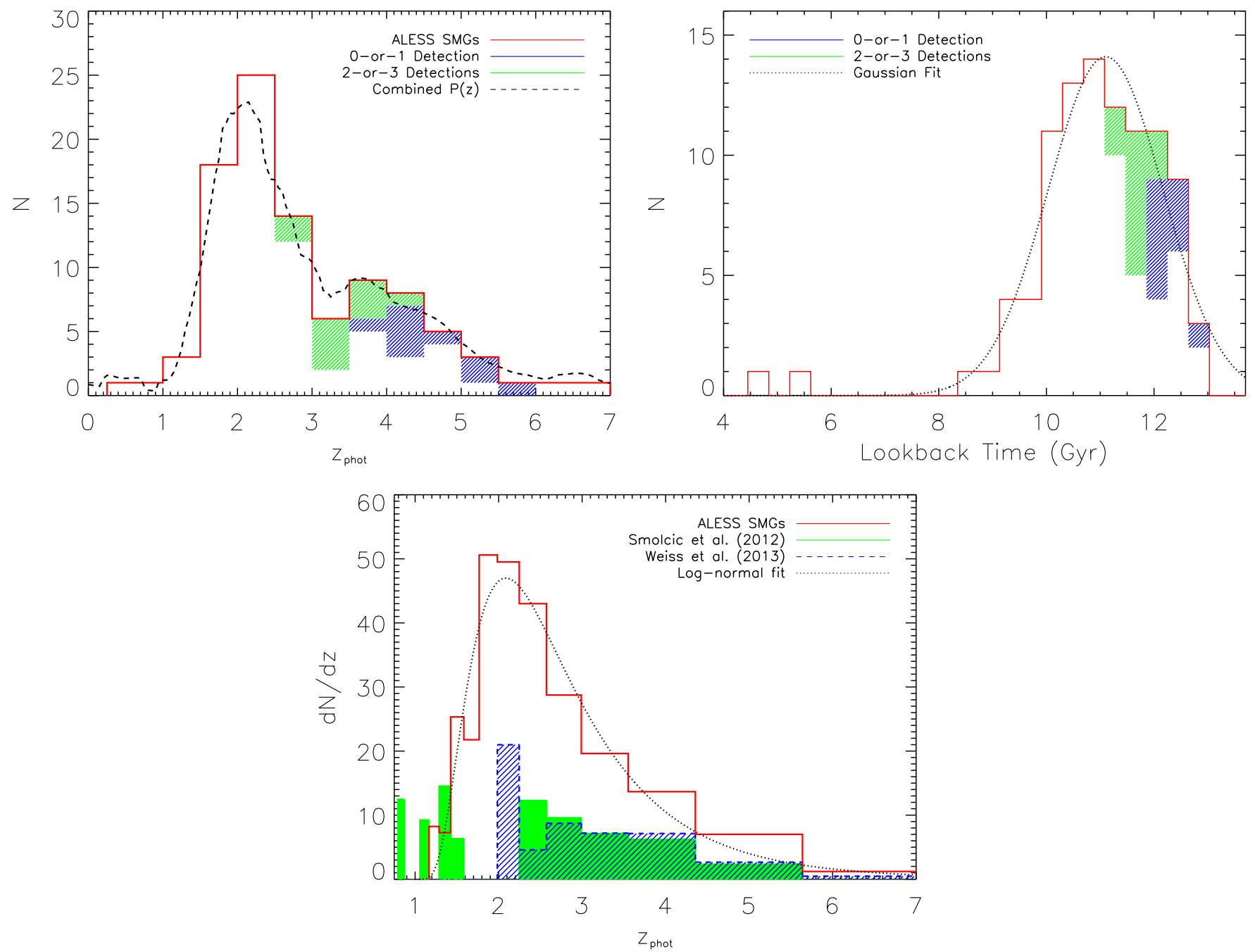

Figure 12. Top Left: Complete redshift distribution of the ALESS SMGs. We assign redshifts to SMGs detected in 0-or-1 and 2-or-3 wavebands by completing the $H$-band absolute magnitude distribution at $z>2.5$, as described in Section 3.2.3. We combine the probability distribution for the photometric redshift of each SMG and overlay this as a dashed line. The combined probability distribution is in close agreement with the shape of the redshift distribution, indicating that the distribution is not sensitive to the uncertainties on individual photometric redshifts, or secondary redshift solutions. Top Right: Complete distribution of ALESS SMGs as a function of time. We find that the distribution is well described by a Gaussian centered at $11.10 \pm 0.05$ Gyr (equivalent to $z=2.6 \pm 0.1$ ), with a width of $1.07 \pm 0.05$ Gyr. Bottom: Redshift distribution of the ALESS SMGs, binned uniformly in time, and normalized by the width of each bin. We find that the redshift distribution is well represented by a lognormal distribution (see Eqn 2) with $\mu=1.53 \pm 0.02$ and $\sigma_{\mathrm{z}}=0.59 \pm 0.01$. For comparison we show the redshift distribution from Smolčić et al. (2012), an interferometric study of 28 millimeter-selected SMGs, containing spectroscopic and photometric redshifts. We also show the spectroscopic redshift distribution from a similar interferometric study of 25 millimeter-selected lensed SMGs from Weiß et al. (2013; hatched), choosing the robust or best-guess redshifts from their analysis. We note that we have included the lensing probability as a function of redshift, given in Weiß et al. (2013), in the distribution. The SMG samples presented here have selection functions that are difficult to quantify (especially the lensed sample of Weiß et al. (2013)), and hence do not have a well-defined survey area. As such, we present the redshift distributions in terms of raw number counts but provide the number of sources in each sample in the legend in the top right. In contrast to these previous studies, the redshift distribution of the ALESS SMGs does not show evidence of a flat distribution between $z \sim 2$ and 6 and displays a clear peak in the distribution at $z=2$.

(A color version of this figure is available in the online journal.)

Smolčić et al. (2012) and hence are indeed likely to lie at high redshifts. We note two interesting features of the Smolčić et al. (2012) redshift distribution: (1) There is a deficit of SMGs at $z \sim 2$, which lies close to the peak of the ALESS SMG redshift distribution (see Figure 12). (2) A further possible discrepancy between the samples is the shape of the distribution from $z=2.5$ to 4.5 , where the ALESS SMG redshift distribution declines whereas the Smolčić et al. (2012) distribution remains relatively flat. However, given the limited number of sources in the comparison, we caution against strong conclusions.

We can also compare to another ALMA sample. Weiß et al. (2013) recently used ALMA to search for molecular emission lines from a sample of 28 strongly lensed SMGs (see also Vieira et al. 2013), selected from observations at $1.4 \mathrm{~mm}$ with the South Pole Telescope (SPT). Given the large beam size $\left(\sim 1^{\prime}\right)$ of the SPT, the sources were also required to be detected at $870 \mu \mathrm{m}$ with LABOCA. Weiß et al. (2013) obtain secure redshifts for 20 SMGs in their sample and provide tentative redshifts, derived from single line identification, for five sources (three sources are not detected in emission). Considering the lower estimates for the tentative redshifts, the sample has a median redshift of $z=3.4 \pm 0.5$, and for the upper limits on the tentative redshifts $z=3.8 \pm 0.4$, with the true median likely lying between the two values. 
The median redshift for the SPT sources is higher than that of the ALESS SMGs, although the two are formally in agreement at a $\sim 2 \sigma$ confidence level. However, the most noticeable discrepancy between the samples lies in the shape of the distributions. Firstly, there are no robust spectroscopic redshift SPT sources at $z<2$, whereas $\sim 25 \%$ of the ALESS SMGs lie at $z_{\text {phot }}<2$, of which seven are spectroscopically confirmed to lie at $z<2$ (see Figure 3; A. L. R. Danielson et al., in preparation). Secondly, the ALESS photometric redshift distribution has a tail to high redshift $\left(z_{\text {phot }} \sim 6\right)$; however, the distribution declines steadily between $z_{\text {phot }}=2$ and 6 . In contrast, the SPT distribution is relatively flat between $z=2$ and 6. As stated by Weiß et al. (2013), their bright $1.4 \mathrm{~mm}$ flux selection criterion, $S_{1.4 \mathrm{~mm}}>20 \mathrm{mJy}$, ensures that they only select lensed sources. This potentially introduces two biases into the redshift distribution: (1) The lensing probability is a function of redshift; for example, from $z=1.5$ (where there are no SPT sources) to $z=6$ the probability of strong gravitational lensing $(\mu \sim 10)$ increases from $P(z)=0.6 \times 10^{-4}$ to $3 \times 10^{-4}$ (i.e., a factor of 5 increase; see Figure 6 from Weiß et al. 2013). In Figure 12 we show the redshift distribution for the SPT sample, corrected by the lensing probability function given in Weiß et al. (2013; see also Hezaveh et al. 2012). We note that this has a significant effect on the shape of the distribution, bringing it into closer agreement with the ALESS sample at $z>2.0$. The weighted median of the corrected Weiß et al. (2013) sample is $z \sim 3.1 \pm 0.3$, which is in agreement with the median redshift of the $z>2.0$ ALESS SMGs of $z_{\text {phot }}=3.0 \pm 0.4$. (2) Evolution in the source size with redshift will affect the lensing magnification, as increasingly compact sources are more highly amplified (Hezaveh et al. 2012; but see discussion in Weiß et al. 2013)

Given the different selection wavelengths between the ALESS SMGs and the SPT sample, as well as the potentially uncertain effects of lensing, we caution against drawing farreaching conclusions between these two redshift distributions. To resolve any tension between these two samples, we require spectroscopic redshifts for an unlensed sample of SMGs, selected at both $870 \mu \mathrm{m}$ and $1.4 \mathrm{~mm}$; however, given the optical properties of these sources, this will only be feasible using a blind redshift search of molecular emission lines, similar to that employed by Weiß et al. (2013).

\subsection{Pairs and Multi-component SMGs}

At least $35 \%$ of the LESS submm sources fragment into multiple SMGs. Using our photometric redshifts, we can now test whether these multiple SMGs are physically associated or simply due to projection effects. In total we derive photometric redshifts for $18 \mathrm{SMG}$ pairs, of which the photometric redshifts of all but one agree at a $3 \sigma$ confidence level. However, as the median combined uncertainty on the photometric redshift of each pair is $\sigma_{z}=0.3$, this simply highlights these large uncertainties. Furthermore, this uncertainty on each pair is similar to the width of the redshift distribution of the whole population, and so we expect SMGs to appear as pairs, irrespective of whether they are associated.

A more sensitive method to test for small-scale clustering of SMGs is to investigate whether there is a significant excess of ALESS SMGs, at similar redshifts, and in the same ALMA map, compared to pairs of SMGs drawn from different ALMA maps. To test for any excess, we initially create random pairs of SMGs, drawn from different ALMA maps, and measure $\Delta z=z_{1}^{\text {phot }}-z_{2}^{\text {phot }}$. We then compare this to the distribution of $\Delta z$ we measure between SMGs in the same ALMA map. To take into account the errors on each photometric redshift, we Monte Carlo the redshift for each SMG within the associated error bar and repeat the entire procedure 1000 times. We identify a tentative excess of $2.8 \pm 1.5$ pairs, from the sample of 18 , at $0<\Delta z<0.5$ in the ALMA maps containing multiple sources. However, this is not a significant result. ${ }^{25}$

The strongest candidates for an associated pair of SMGs are ALESS 55.1 and 55.5. These SMGs are separated by $\sim 2$ ", have merged $870 \mu \mathrm{m}$ emission, and straddle a single optical-NIR counterpart; the photometric redshift of this source indicates that it is not a lensing system. We note that the photometry for these SMGs is drawn from the same optical-NIR source. A further two LABOCA sources, LESS 67 and LESS 116, fragment into multiple ALESS SMGs with similarly small on-sky separations $\left(<3^{\prime \prime}\right)$; however, we cannot verify whether they are physically associated.

\subsection{Stellar Masses}

We estimate stellar masses for the ALESS SMGs from their absolute $H$-band magnitudes, which we note are calculated from the best-fit SED and take into account the effects of the $K$-correction. We select this waveband as a compromise between limiting the effects of dust extinction (the correction decreases with increasing wavelength) and the potential contribution of thermally pulsating asymptotic giant branch (TP-AGB) stars (which increases at shorter wavelengths [Henriques et al. 2011]).

The median absolute $H$-band magnitude for the 77 ALESS SMGs detected in $>4$ wavebands is $-24.56 \pm 0.15$. As discussed in Section 3, by assuming that the ALESS SMGs detected in $<4$ wavebands are missed due to our photometric selection limits, we can complete the $M_{\mathrm{H}}$ distribution by enforcing the condition that the distribution is not bimodal. Using the complete $M_{\mathrm{H}}$ distribution, we measure a median absolute $H$-band magnitude for the ALESS SMGs of $-24.33 \pm 0.15$. We note that this is corrected for a median reddening of $A_{V}=1.7 \pm 0.1$ (Section 3.3). The median value of $M_{\mathrm{H}}$ for the ALESS SMGs is in agreement with previous work by Hainline et al. (2011), who measure a median $M_{\mathrm{H}}=-24.45 \pm 0.20$ for the stellar emission from a sample of 65 spectroscopically confirmed, radio-identified SMGs from the Chapman et al. (2005) sample.

To convert these absolute $H$-band magnitudes to stellar masses, we must next adopt a mass-to-light ratio. As we discussed in Section 3, the SFHs for the ALESS SMGs are highly degenerate, and it is not possible to accurately distinguish between the model SFHs. To determine a mass-to-light ratio, we therefore consider the range spanned by the best-fit Burst and Constant SFHs (the two extremes of SFH we consider). We use the Bruzual \& Charlot (2003) simple stellar populations (SSPs) to construct an evolved spectrum from the best-fit constant and burst SFHs for each SMG, and measure the absolute $H$-band magnitude. ${ }^{26}$ We then define the stellar mass as the total mass in stars and stellar remnants, using STARBURST99 to determine the mass lost due to winds and supernovae (Leitherer et al. 1999; Vázquez \& Leitherer 2005; Leitherer et al. 2010).

The median mass-to-light ratio for the ALESS SMGs is $M / L_{H}=0.08 \pm 0.02$ for the Burst SFH and $M / L_{H}=$ $0.25 \pm 0.05$ for the Constant SFH; however, we caution that the

\footnotetext{
25 We note that including sources from the Supplementary ALESS catalog in this analysis does not increase the significance of the result.

26 We enforce the condition that the age of the star formation event is $20 \mathrm{Myr} \leqslant t_{\text {age }} \leqslant 1$ Gyr.
} 
mass-to-light ratios between the Burst and the Constant SFH solutions for individual SMGs vary by $>3 \times$ for $\sim 40 \%$ of the sample. Nevertheless, we apply the best-fit mass-to-light ratios for each of the 77 ALESS SMGs detected in $>3$ wavebands to their dust-corrected absolute $H$-band magnitudes and determine median stellar masses of $M_{\star}=(7.4 \pm 1.0) \times 10^{10} M_{\odot}$ for the Burst SFH, $M_{\star}=(9.2 \pm 0.8) \times 10^{10} M_{\odot}$ for the Constant SFH, and $M_{\star}=(8.9 \pm 1.4) \times 10^{10} M_{\odot}$ if we take the average of the mass estimates for each SMG. For the 19 SMGs detected in $<4$ wavebands we do not have sufficient information on the SFH to determine a mass-to-light ratio. If we adopt the median mass-to-light ratio for the detected SMGs, the stellar mass of the non-detected SMGs is $M_{\star}=(2.9 \pm 0.4) \times 10^{10} M_{\odot}$ for the Burst SFH, $M_{\star}=(8.5 \pm 1.3) \times 10^{10} M_{\odot}$ for the Constant SFH, and $M_{\star}=(5.7 \pm 0.8) \times 10^{10} M_{\odot}$ for the average of the mass estimates. Combining the samples, we derive a median stellar mass for the 96 ALESS SMGs of $M_{\star}=(8 \pm 1) \times 10^{10} M_{\odot}$, when taking the mass as the average of the Burst and Constant values. We note that all the stellar masses quoted here are for a Salpeter initial mass function (IMF), and the median mass-to-light ratio is $M / L_{H}=0.15 \pm 0.01^{27}$ (the average mass-to-light ratio between a 100 Myr Burst and Constant SFH is $M / L_{H}=0.14$ ).

The median stellar mass for the ALESS SMGs is lower than that found for the C05 sample of SMGs by Hainline et al. $\left(2011 ; M_{\star}=1.6 \pm 0.3 \times 10^{11} M_{\odot}\right.$; see also Michałowski et al. 2010: $\left.M_{\star}=3.5 \times 10^{11} M_{\odot}\right)$. Given the uncertainty surrounding stellar mass estimates, it is more informative to compare the absolute $H$-band magnitudes of the ALESS SMGs to the C05 sample. As stated earlier, these are in agreement, and so any difference in the median stellar mass is due to differences in the mass-to-light ratios adopted. We note that the C05 sample of SMGs has significant contamination in $M_{H}$ due to AGN activity (Hainline et al. 2011), which we do not see for the ALESS SMGs. However, the median $M_{H}$ and masses for the C05 SMGs quoted here are corrected for that AGN contamination.

Although we highlight that the stellar masses for the ALESS SMGs are highly uncertain, we can crudely test their accuracy by comparing them to the dynamical masses and $\mathrm{CO}$-derived gas masses of similar SMGs. Bothwell et al. (2013) recently obtained observations of ${ }^{12} \mathrm{CO}$ emission from $32 \mathrm{SMGs,} \mathrm{drawn}$ from the C05 sample. These SMGs have typical single-dishderived $870 \mu \mathrm{m}$ fluxes of 4-20 mJy and were found to have a median gas mass of $M_{\text {gas }}=(3.5 \pm 1.1) \times 10^{10} M_{\odot}$. We note that Swinbank et al. (2014) used the dust masses of the ALESS SMGs to derive a median gas mass of $M_{\text {gas }}=$ $4.2 \pm 0.4 \times 10^{8} M_{\odot}$, comparable to the result from Bothwell et al. (2013). Combining the median gas mass from Bothwell et al. (2013) with the median stellar mass of the ALESS SMGs, and assuming a dark matter contribution of $\sim 25 \%$, suggests that SMGs have typical dynamical masses of $\sim(1-2) \times 10^{11} M_{\odot}$. Crucially, our estimate of the dynamical mass is consistent with spectroscopic studies of resolved $\mathrm{H} \alpha$ or ${ }^{12} \mathrm{CO}$ emission lines, which demonstrate that SMGs typically have dynamical masses of $(1-2) \times 10^{11} M_{\odot}$ (Swinbank et al. 2004; Alaghband-Zadeh et al. 2012; Bothwell et al. 2013), inside a $5 \mathrm{kpc}$ radius.

\subsection{Evolution of SMGs: $z=0$}

We now investigate the possible properties of the descendants of the ALESS SMGs at the present day by modeling

\footnotetext{
27 The mass-to-light ratio for the instantaneous burst SFH is sensitive to changes on the order of $\sim 10 \mathrm{Myr}$, and over the range 10-40 Myr it varies from $M / L_{H} \sim 0.02$ to 0.1 . However, when considering the range 10-40 Myr the median stellar mass remains stable at $M_{\star} \sim(8 \pm 1) \times 10^{10} M_{\odot}$.
}

how much their $H$-band luminosity will fade between their observed redshift and the present redshift. First, we must make assumptions about the future evolution of the ALESS SMGs, the most crucial of which is the duration of the SMG phase. As stated in Section 4.3, based on existing CO studies of SMGs the ALESS SMGs are likely to have a median gas mass of $M_{\text {gas }} \sim(4 \pm 1) \times 10^{10} M_{\odot}$, and from Swinbank et al. (2014) they have a median SFR of $840 \pm 120 M_{\odot} \mathrm{yr}^{-1}$ for a Salpeter IMF. If the SFR remains constant and all the gas is converted into stars, this suggests that the SMG phase has a maximum duration on the order of $100 \mathrm{Myr}$ (see also Swinbank et al. 2006; Hainline et al. 2011; Hickox et al. 2012).

To measure the change in $H$-band luminosity of the ALESS SMGs, we use the Bruzual \& Charlot (2003) SSPs to model the SED evolution. On average we are seeing each SMG midway through its burst, and so, for an SMG duration of $100 \mathrm{Myr}$, we calculate the fading in $L_{H}$ between $50 \mathrm{Myr}$ into the burst and the required age at the present day. We note that this assumes that the contribution to the fading from a pre-burst stellar population is negligible and that each SMG undergoes only a single burst.

The ALESS SMGs represent a complete survey over $0.25 \mathrm{deg}^{2}$, and so we can also calculate their co-moving space density. We first extrapolate the ALESS sample to $S_{870} \geqslant 1 \mathrm{mJy}$, using the ALESS SMG number counts from Karim et al. (2013), noting that we again make the assumption that there is no dependence of $M_{H}$ on $S_{870}$. We also apply a factor of two correction to the number counts to account for the under-density of SMGs in the ECDFS (see Weiß et al. 2009). As the SMG phase has a finite duration, we duty-cycle-correct the number density following

$$
\phi_{\mathrm{D}}=\rho_{\mathrm{SMG}}\left(t_{\mathrm{obs}} / t_{\text {burst }}\right),
$$

where $\phi_{\mathrm{D}}$ is the comoving space density of SMG descendants, $\rho_{\mathrm{SMG}}$ is the observed space density of ALESS SMGs, $t_{\mathrm{obs}}$ is the duration of the epoch over which we observe the SMGs, and $t_{\text {burst }}$ is the duration of the SMG phase. We estimate $t_{\mathrm{obs}}$ from the 10th-90th percentiles of the redshift distribution, $1.6<z<4.5$, and as stated earlier we assume that the SMG phase has a duration of 100 Myr. Taking these corrections into account, we estimate that the volume density of the descendants of $S_{870} \geqslant 1$ mJy SMGs is $\sim(1.4 \pm 0.4) \times 10^{-3} \mathrm{Mpc}^{-3}$.

It has been suggested that SMGs may be the progenitors of local elliptical galaxies (e.g., Lilly et al. 1999; Genzel et al. 2003; Blain et al. 2004; Swinbank et al. 2006; Tacconi et al. 2008; Swinbank et al. 2010a). We now test the relation of the descendants of the ALESS SMGs to local ellipticals using a morphologically classified sample of elliptical galaxies, taken from the Padova Millennium Galaxy and Group Catalog (PM2GC; Calvi et al. 2011, 2013). This catalog represents a volume-limited survey $(z=0.03-0.1)$ over $38 \mathrm{deg}^{2}$, with morphologies determined by an automatic tool that mimics a visual classification (Calvi et al. 2012; see also Fasano et al. 2012). These galaxies were observed in the $Y, H$, and $K$ bands by the UKIDSS Large Area Survey (Lawrence et al. 2007), and we derive absolute $H$-band magnitudes from the recent data release (Lawrence et al. 2012). This comparison sample of local elliptical galaxies has a median redshift of $z=0.08$, median absolute $H$-band magnitude of $M_{H}=-21.1 \pm 0.1$, and a space density of $(2.0 \pm 0.1) \times 10^{-3} \mathrm{Mpc}^{-3}$.

Using the observed redshift of the SMGs and our adopted $\mathrm{SFH}$, we individually fade each ALESS SMG to $z=0.08$ and estimate a median faded absolute $H$-band magnitude of $M_{H}=-21.2 \pm 0.2$. We show the "faded" distribution in Figure 13, where we see very good agreement with absolute 

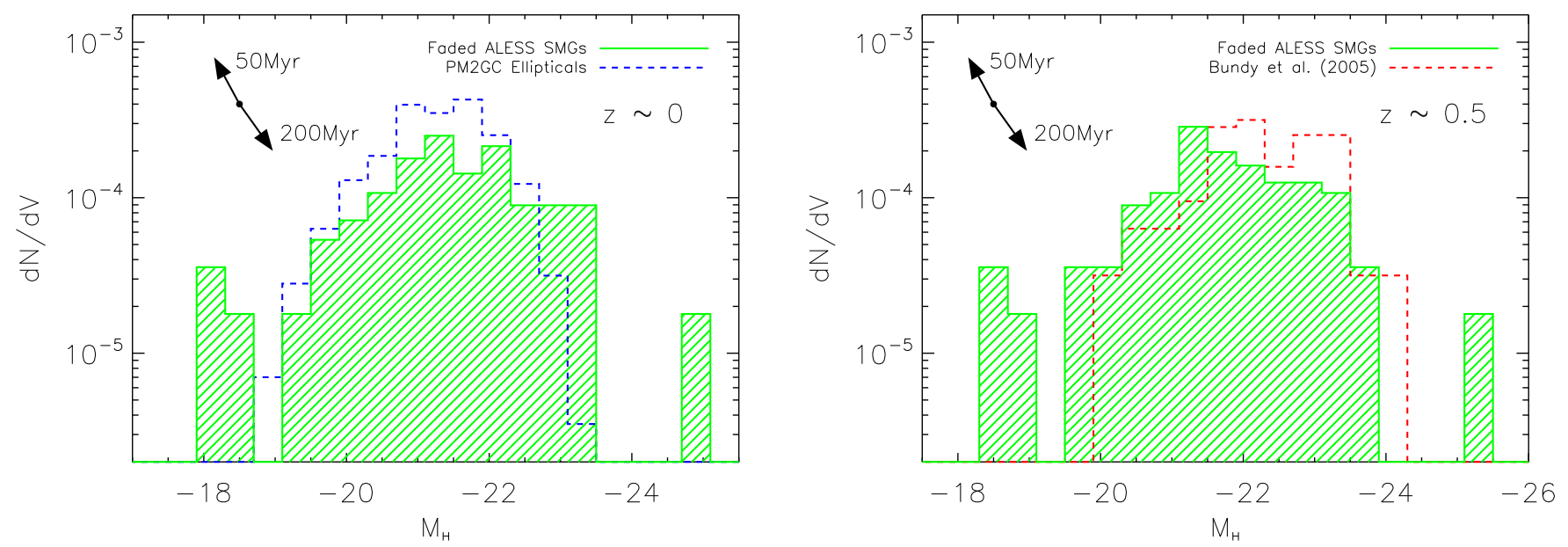

$\mathrm{M}_{\mathrm{H}}$

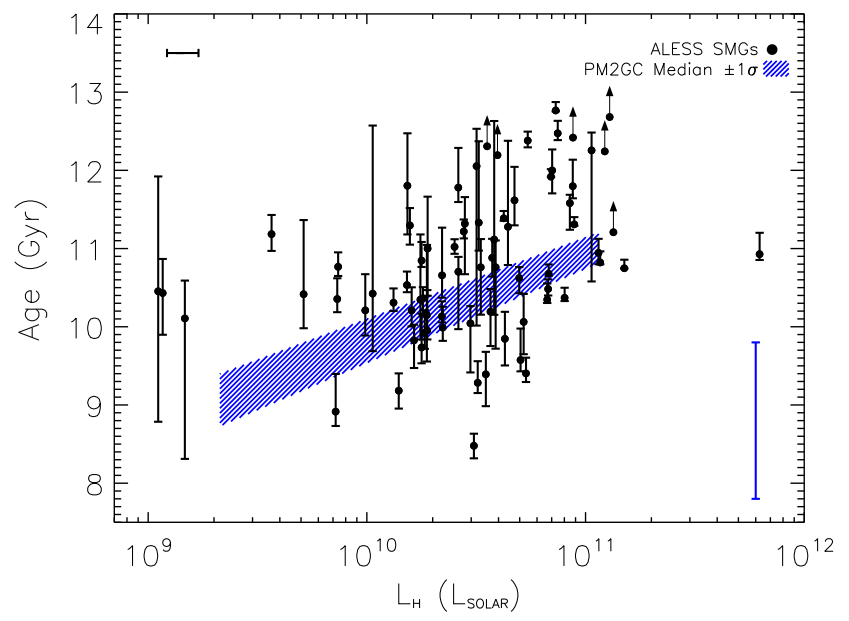

Figure 13. Top Left: Absolute $H$-band magnitude distribution of the ALESS SMGs, faded to the present day assuming a $100 \mathrm{Myr}$ burst duration SFH. We adopt the SMG number counts from Karim et al. (2013) to extrapolate the ALESS SMG sample to $1 \mathrm{mJy}$, and duty-cycle-correct the volume density. In the upper left, vectors indicate the effect of adopting either a $50 \mathrm{Myr}$ or $200 \mathrm{Myr}$ burst. In comparison, we show the absolute $H$-band magnitude distribution of a morphologically classified, volume-limited sample of elliptical galaxies over the redshift range 0.03-0.1 (PM2GC; Calvi et al. 2011, 2013). We conclude good agreement in both typical luminosity range and space density of faded SMGs to local ellipticals. Top Right: We again show the ALESS SMGs, faded to $z=0.5$. We compare this to an absolute $H$-band magnitude distribution for a morphologically classified sample of elliptical galaxies at $z \sim 0.5$ (Bundy et al. 2005). We find that the number densities of the SMGs and intermediate-redshift ellipticals are in agreement; however, the ALESS SMGs are on average $\sim 0.5$ mag fainter. Bottom: Comparison of the mass-weighted ages of the PM2GC sample of elliptical galaxies to the current look-back age of the ALESS SMGs. The error bar in the bottom right of the figure shows the typical mass-weighted-age error at these old ages, as derived from the spectrophotometric modeling (Poggianti et al. 2013). Despite the large systematic uncertainty, the PM2GC ellipticals have mass-weighted ages that are in broad agreement with the ALESS SMGs. This is consistent with a simple evolutionary model where SMGs are the progenitors of local elliptical galaxies. The typical error in $L_{H}$ for the ALESS SMGs is shown in the upper left of the figure.

(A color version of this figure is available in the online journal.)

$H$-band magnitude distribution of the PM2GC ellipticals. As stated earlier, we estimate that the space density of the descendants of ALESS SMGs is $(1.4 \pm 0.4) \times 10^{-3} \mathrm{Mpc}^{-3}$, similar to the PM2GC ellipticals, $(2.0 \pm 0.1) \times 10^{-3} \mathrm{Mpc}^{-3}$. We note that both the fading correction in $M_{H}$ and the number density of the SMGs are dependent on the duration of the SMG phase. If we instead adopt a burst of 50 or $200 \mathrm{Myr}$ duration, then the median absolute $H$-band magnitude is $M_{H}=-20.9 \pm 0.2$ or $M_{H}=-21.7 \pm 0.2$, and the number density is $(3 \pm 1) \times 10^{-3}$ or $(0.7 \pm 0.2) \times 10^{-3} \mathrm{Mpc}^{-3}$, respectively, and these changes are shown by vectors in Figure 13 .

We note that if the burst duration is indeed $\gtrsim 200 \mathrm{Myr}$, then $>10 \%$ of the SMG descendants would have an $H$-band absolute magnitude brighter than the brightest elliptical in the PM2GC sample. This excess of bright galaxies assumes that SMGs undergo no future interactions, i.e., minor mergers, or subsequent star formation, which would only act to increase the total absolute $H$-band magnitude and thus make the discrepancy larger. We suggest that this makes burst durations of $>200 \mathrm{Myr}$ unlikely. For our estimated burst duration of $100 \mathrm{Myr}$ the space density of SMGs is lower than local ellipticals, indicating that the SMG phase could be $<100$ Myr. A burst duration shorter than 100 Myr would make the descendants of the ALESS SMGs fainter than the $z=0$ elliptical sample but have a larger space density. If we consider a burst duration of $50 \mathrm{Myr}$, then a drymerger fraction of two-thirds would bring the number density into agreement with the PM2GC ellipticals, and the resulting median $M_{H}$ of the SMGs to $M_{H}=21.4 \pm 0.1$.

We now consider two further tests of this evolutionary model. First, we consider the mass-weighted stellar ages of the PM2GC ellipticals, calculated with a spectrophotometric model that finds the combination of SSP synthetic spectra that best fits the observed spectroscopic and photometric features of each galaxy (Poggianti et al. 2013). As can be seen in Figure 13, these appear broadly consistent with the ages of the ALESS SMGs. The PM2GC ellipticals have a mass-weighted stellar 
age of $\sim 10$ Gyr $(z \sim 2)$ but with a systematic uncertainty of \pm 2 Gyr (Poggianti et al. 2013), compared to the median age of the ALESS SMGs of $11.1 \pm 0.1$ Gyr. This might indicate that the bulk of stellar mass in the ellipticals formed later than the current redshift of the ALESS SMGs; however, given the systematic uncertainties and the difficulty in age-dating very old stellar populations, we find the similarity in the ages striking.

As a second comparison, we consider the mass of the dark matter halos in which the PM2GC ellipticals reside. We use the halo mass catalogs from Yang et al. (2005), and find that these ellipticals have a typical halo mass of $0.5 \times 10^{13} M_{\odot}$ with a $1 \sigma$ range of $(0.1-8) \times 10^{13} M_{\odot}$. This is consistent with the typical halo masses of SMG descendants $\left(3 \times 10^{13} M_{\odot}\right.$ with a $1 \sigma$ range of $(0.9-7) \times 10^{13} M_{\odot}$; Hickox et al. 2012), although there is clearly a large amount of scatter.

We conclude that by assuming a simple scenario where an SMG undergoes a star formation event with a duration of $100 \mathrm{Myr}$, at a constant SFR, and then evolves passively, we determine that the median absolute $H$-band magnitude and number density of the ALESS SMGs are in good agreement with those of $z \sim 0$ ellipticals. We also find that the shape of the absolute $H$-band distribution (Figure 13), the mass-weighted stellar ages, and the halo masses of local ellipticals are in good agreement with those predicted for the descendants of SMGs, suggesting that within this simple model SMGs are sufficient to explain the formation of most local elliptical galaxies brighter than $M_{H} \sim-18.5$.

\subsection{Evolution of SMGs: Intermediate-redshift tests}

We now consider whether intermediate-redshift populations agree with the toy model proposed here. We use a catalog from Bundy et al. (2005), which provides morphological classifications for $z<22.5 \mathrm{mag}$ galaxies in the GOODS-South field. These galaxies are covered by the photometry employed in Section 2, and so we can derive a photometric redshift and measure an absolute $H$-band magnitude for each source from SED fitting in the same manner as the ALESS SMGs. We select galaxies from the catalog that are visually classified as ellipticals and with a photometric redshift $0.4<z_{\text {phot }}<0.6$ (the mid-point in time of evolution from an SMG phase to the local universe). The final sample has a median absolute $H$-band magnitude of $M_{H}=-22.0 \pm 0.2$ and a number density of $(1.6 \pm 0.2) \times 10^{-3} \mathrm{Mpc}^{-3}$. The number density of the ALESS SMGs, $(1.4 \pm 0.4) \times 10^{-3} \mathrm{Mpc}^{-3}$, is in agreement with the intermediate population of elliptical galaxies; however, fading the absolute $H$-band magnitudes of the ALESS SMGs to $z \sim 0.5$, we find that they are marginally fainter at $M_{H}=-21.5 \pm 0.2$ (see Figure 13). Given the difficulty in morphological classification at $z \sim 0.5$ and the small number of sources in each sample, we caution against drawing strong conclusions from this result. However, it does indicate that the ALESS SMGs are broadly consistent with $z \sim 0.5$ elliptical galaxies.

Recently, NIR spectroscopy with the Wide-Field Camera 3 (WFC3) on the Hubble Space Telescope (HST) has been used to estimate the age of the stellar populations in quiescent spheroidal galaxies at $1.5<z<2$, the likely progenitors of local ellipticals. In particular, Whitaker et al. (2013) recently used this technique to estimate stellar ages for a sample of 171 quiescent galaxies at $1.4<z<2.2$, with stellar masses $M_{\star} \gtrsim 5 \times 10^{10} M_{\odot}$ (similar to the ALESS SMGs). Whitaker et al. (2013) divide their quiescent sample into blue (34) and red (137) subsets, based on $U_{-}, V$-, and $J$-band colors, and fit absorption-line models to the stacked spectra of each subset, deriving median stellar ages of $0.9_{-0.1}^{+0.2}$ and $1.6_{-0.4}^{+0.5} \mathrm{Gyr}$ for the blue and red subsets, respectively. We use a weighted average of the number of galaxies in each subset, to determine that the complete sample has a median stellar age of $\sim 1.4 \mathrm{Gyr}$, at a median redshift of $z \sim 1.7$ (see Figure 4 in Whitaker et al. 2013). Combining the median stellar age and redshift of these post-starburst galaxies suggests that they formed at $z \sim 2.6$ (see also Bedregal et al. 2013) This is consistent with the toy model proposed earlier where the ALESS SMGs, with a median redshift of $z_{\text {phot }}=2.5 \pm 0.2$, form the majority of their stellar mass in a single burst at $z \sim 2.5$ and do not undergo subsequent periods of significant star formation.

\subsection{Evolution of SMGs: Black Hole Masses}

Finally, given the apparent link between SMGs and elliptical galaxies at $z \sim 0$, we now investigate whether their black hole masses are consistent with our toy evolutionary model. To estimate black hole masses for the ALESS SMGs, we use their X-ray properties, presented in Wang et al. (2013). However, only 10 ALESS SMGs are detected at X-ray energies, and so we must first consider whether these SMGs are representative of the entire sample.

In our approach we will assume that the black hole masses of the X-ray-detected SMGs are related to the total stellar mass of the galaxy; however, in our initial analysis we will use the $H$-band luminosity as a proxy for stellar mass. To account for the positive $K$-correction in the X-ray band, we only consider SMGs in the redshift range $z=0-2.5$, which includes seven X-raydetected SMGs. These SMGs have a median $H$-band luminosity of $L_{H}=(1.1 \pm 0.3) \times 10^{12} L_{\odot}$, which is brighter than for the population as a whole $\left(L_{H}=(0.5 \pm 0.1) \times 10^{12} L_{\odot}\right.$; Figure 9); a two-sided K-S test returns a $3 \%$ probability that they are drawn from the same parent distribution. If we instead split the ALESS SMG sample at the median $H$-band luminosity, $L_{H}=0.5 \times 10^{12} L_{\odot}$, then the X-ray non-detected SMGs at $L_{H}>0.5 \times 10^{12} L_{\odot}$ have a median $H$-band luminosity of $L_{H}=(1.0 \pm 0.2) \times 10^{12} L_{\odot}$ and a two-sided K-S test returns a probability of $85 \%$ that the X-ray-detected and non-detected SMGs with $L_{H}>0.5 \times 10^{12} L_{\odot}$ are drawn from the same parent distribution (see also Wang et al. 2013).

A possible explanation for the higher $H$-band luminosities of the X-ray-detected SMGs is that they suffer contamination in $L_{H}$ from an AGN power-law component (see Hainline et al. 2011). However, to bring the $H$-band luminosity of the $\mathrm{X}$-ray-detected SMGs into agreement with the complete sample requires a power-law fraction of $\sim 50 \%$, which is not seen in the SEDs of the ALESS SMGs (see Appendix A).

We now investigate whether these X-ray-detected SMGs are preferentially detected in X-ray emission due to higher SFRs. Using the FIR-derived SFRs for the ALESS SMGs from Swinbank et al. (2014), we find that the X-ray-detected SMGs have a median SFR of $\mathrm{SFR}_{\mathrm{FIR}}=570 \pm 140 M_{\odot} \mathrm{yr}^{-1}$, which is consistent with the X-ray non-detected, $L_{H}>0.5 \times 10^{12} L_{\odot}$ SMGs, which have a median $\mathrm{SFR}_{\mathrm{FIR}}$ of $590 \pm 130 M_{\odot} \mathrm{yr}^{-1}$. We note that the median SFR FIR of the $L_{H}<0.5 \times 10^{12} L_{\odot}$ SMGs is $220 \pm 40 M_{\odot} \mathrm{yr}^{-1}$, a factor of $\sim 2.5 \times$ lower. This suggests that the potential X-ray emission from star formation is similar for the X-ray-detected and non-detected SMGs with $L_{H}>0.5 \times 10^{12} L_{\odot}$ and is consistent with the results from Wang et al. (2013), who argue for dominant AGN contributions to the X-ray emission of the X-ray-detected SMGs. 
These results suggest that the X-ray-detected SMGs have $H$-band luminosities and SFRs comparable to the $L_{H}>$ $0.5 \times 10^{12} L_{\odot}$ SMGs. As we demonstrated in Section 3.2.2, the X-ray-detected SMGs are also indistinguishable from the $\mathrm{X}$-ray non-detected SMGs in terms of IRAC flux ratios, and so combining all these results, we conclude that, other than in $\mathrm{X}$-ray emission, the X-ray-detected SMGs are not distinguishable from average $L_{H}>0.5 \times 10^{12} L_{\odot}$ SMGs. Instead, we propose that SMGs with $L_{H} \lesssim 0.5 \times 10^{12} L_{\odot}$ are not detected at X-ray energies due to the selection limits on the X-ray data. As the median $H$-band luminosity of the ALESS SMGs with $L_{H}<0.5 \times 10^{12} L_{\odot}$ is a factor of $3.5 \times$ lower than the brighter half of the sample, then, under the simple assumption that $L_{X}$ and $L_{H}$ represent $M_{\mathrm{BH}}$ and $M_{\star}$, this suggests that X-ray data a factor of $\sim 3.5 \times$ deeper are required to detect these SMGs down to the same Eddington ratio as the X-ray-detected SMGs.

Although the X-ray-detected SMGs only appear representative of the ALESS SMGs above $L_{H}>0.5 \times 10^{12} L_{\odot}$, we can still estimate their black hole masses and hence their relation to the black hole masses of local ellipticals. The 10 ALESS SMGs detected at X-ray energies have a median absorption-corrected $X$-ray luminosity of $\log L_{0.5-8 \mathrm{keV} \text {,corr }}=43.3 \pm 0.4 \mathrm{erg} \mathrm{s}^{-1}$. We initially convert the luminosity from $0.5-8 \mathrm{keV}$ to $2-10 \mathrm{keV}$, dividing through by a conversion factor of 1.21 , and estimate a bolometric luminosity following $L_{\text {bol }}=35 \times L_{2-10}$ (Alexander et al. 2008). We adopt an Eddington ratio of $\eta=0.2$, which was calculated for a small number of SMGs with direct black hole mass measurements (Alexander et al. 2008), and from this estimate we calculate a median black hole mass of $M_{\mathrm{BH}} \sim\left(3_{-1}^{+3}\right) \times 10^{7} M_{\odot}$. We note that our uncertainty does not include any error in the Eddington ratio or the bolometric luminosity conversion and assumes that the average Eddington ratio for these SMGs is consistent with the SMGs in Alexander et al. (2008).

Using the black hole masses and stellar masses of the X-raydetected ALESS SMGs, we can estimate the growth required to match the black holes in local ellipticals. The X-ray-detected SMGs have $M_{\mathrm{BH}} / M_{\star}=0.2_{-0.1}^{+0.2} \times 10^{-3}$ and require $\sim 9 \times$ growth of their black hole masses, at fixed stellar mass, to match the local relation $\left(M_{\mathrm{BH}} / M_{\star}=1.7 \pm 0.4 \times 10^{-3}\right.$ at these masses; Häring \& Rix 2004). If we assume that the SMG lifetime is $\sim 100 \mathrm{Myr}$, and that we are seeing each SMG on average halfway through the burst, then following Eqn 10 from Alexander \& Hickox (2012), and assuming $\eta=0.2$, the supermassive black hole (SMBH) will have grown by $\sim 20 \%$ at the end of the SMG phase. However, a further factor of $\sim 7 \times$ growth is still required to match the local $M_{\star}-M_{\mathrm{BH}}$ relation. It has been speculated that this growth may come in the form of a QSO phase (e.g., Sanders et al. 1988; Coppin et al. 2008; Simpson et al. 2012), during which the SMBH would grow at approximately the Eddington limit. If we assume that all of the remaining SMBH growth occurs in a QSO phase, then the duration of this phase must be $\sim 100$ Myr.

A lifetime of $100 \mathrm{Myr}$ for a QSO phase is high but not unreasonable (Martini \& Weinberg 2001); however, it is highly unlikely that the accretion is Eddington limited for the entirety of this period (McLure \& Dunlop 2004; Kelly et al. 2010). If the accretion is not Eddington limited during this phase, then this analysis suggests that we have either underestimated the black hole masses or overestimated the stellar masses in these SMGs. Indeed, when estimating black hole masses for the $\mathrm{X}$-ray-detected SMGs, we have made assumptions on the current Eddington ratio, $\eta$, and the conversion from X-ray luminosity to a bolometric luminosity, both of which have significant uncertainties. The black hole masses we estimate are inversely proportional to the initial Eddington ratio, and it is feasible that this is lower than the value we adopted $(\eta=0.2$; Alexander et al. 2008). Due to the uncertainties surrounding our estimate of the median black hole mass, we simply conclude that the SMBHs in these SMGs are likely to require an extended period of black hole growth after the SMG phase, and that this is most likely to occur during a high accretion rate QSO phase.

\section{SUMMARY AND CONCLUSIONS}

In this paper we have presented a multi-wavelength study of the first large sample of $870 \mu \mathrm{m}$ selected SMGs with unambiguous identifications based on ALMA interferometry. Crucially, these precise identifications, at the same wavelength as used for the original single-dish survey, mean that our analysis is free from the mis-identification and incompleteness associated with the use of radio-mid-infrared proxies to locate the counterparts of single-dish-identified submm sources (see discussion in Hodge et al. 2013). The main conclusions from our work are as follows:

1. We measure aperture photometry in 19 wavebands for 96 ALESS SMGs (Hodge et al. 2013). From this initial sample 77 SMGs are detected in $\geqslant 4$ wavebands and have sufficient photometry to derive photometric redshifts from SED fitting. These 77 SMGs have a median redshift of $z_{\text {phot }}=2.3 \pm 0.2$, with a $1 \sigma$ spread of $z_{\text {phot }}=1.8-3.5$.

2. Nineteen SMGs in our sample have insufficient photometry to derive photometric redshifts. We initially divide these sources into subsets detected in 0-or-1 and 2-or-3 wavebands and test whether they are real or spurious by stacking their emission in other wavebands. Detections at 250, 350, and $500 \mu \mathrm{m}$ for both subsets confirm that these are typically FIR-bright sources on average.

3. We use the distribution of absolute $H$-band magnitudes at $z<2.5$ to measure the incompleteness in the distribution at $z>2.5$, and we use this to estimate redshifts for the 19 ALESS SMGs detected in $<4$ wavebands. We estimate the median redshifts for the SMGs detected in 2-or-3 and 0 -or- 1 wavebands as $z \sim 3.5$ and $z \sim 4.5$, respectively.

4. The redshift distribution for the complete sample of 96 ALESS SMGs has a median redshift of $z_{\text {phot }}=2.5 \pm 0.2$ with $35 \% \pm 5 \%$ of SMGs lying at $z>3$. In terms of their look-back age, the distribution is well fit by a Gaussian distribution centered at $11.1 \pm 0.1 \mathrm{Gyr}$, and with a $1 \sigma$ width of $1.1 \pm 0.1 \mathrm{Gyr}$.

5. We compare the redshift distribution of the ALESS SMGs to recent interferometric observations of smaller samples of millimeter-selected SMGs from Smolčić et al. (2012) and Weiß et al. (2013). The median redshift of the SMGs presented in Smolčić et al. (2012) is $z=2.6 \pm 0.4$, in agreement with the ALESS SMGs; however, the median redshift of millimeter-selected, lensed SMGs in Weiß et al. (2013) is considerably higher, $z \sim 3.6 \pm 0.4$, although correcting for lensing effects reduces this to $z \sim 3.1 \pm 0.3$. Due to differences in the selection wavelength and the difficulties in accurately constraining the lensing selection function, we caution against strong conclusions drawn from the discrepancy in the median redshifts of the ALESS SMGs and the SMGs presented in Weiß et al. (2013). We note that in contrast to both of these previous studies, which 
have suggested that the redshift distribution of SMGs is flat above $z \sim 3$, the ALESS redshift distribution peaks at $z \sim 2.5$ and declines steadily toward high redshift $(z>4)$.

6. Nineteen of the 86 ALMA maps employed in our analysis do not contain an SMG with an $870 \mu \mathrm{m}$ flux density $>1.4$ mJy. Karim et al. (2013) and Hodge et al. (2013) propose that this is due to the original LABOCA submm source fragmenting into a number of faint SMGs, and so we measure the number density and redshift distribution of $3.6 \mu \mathrm{m}$ sources in these "blank" maps and compare to the redshift distribution in the field. We identify an excess of sources at $z \sim 1-3$, indicating that, on average, these "blank" maps contain multiple SMGs below our detection threshold. We stack the ALMA $870 \mu \mathrm{m}$ maps at the position of these $3.6 \mu \mathrm{m}$ sources and measure an average flux of $0.36 \pm 0.09 \mathrm{mJy}$ per source and a total contribution per map of $0.76 \pm 0.19 \mathrm{mJy}$. Although this is not sufficient to explain the difference in total flux measured by LABOCA and ALMA in these regions, it suggests that there are faint SMGs in these "blank" maps, which lie at a similar redshift to the $S_{870}$-brighter ALESS SMGs.

7. Using the complete $M_{H}$ distribution for our sample, we derive a median stellar mass of $(8 \pm 1) \times 10^{10} M_{\odot}$ for the ALESS SMGs, but caution that due to the unconstrained SFHs and hence mass-to-light ratios, this is subject to significant systematic uncertainties (at least $\sim 5 \times$ ).

8. We investigate the possible properties of the descendants of the ALESS SMGs at the present day. Fading the $H$-band luminosities of the ALESS SMGs, assuming an SFH consisting of a 100 Myr burst with constant SFR, we show that the present-day descendants will have an absolute $H$-band magnitude of $M_{H} \sim-21.2 \pm 0.2$ and a space density of $(1.4 \pm 0.4) \times 10^{-3} \mathrm{Mpc}^{-3}$. These properties are in good agreement with those of local elliptical galaxies, derived from the volume-limited PM2GC survey, which have a median absolute $H$-band magnitude of $M_{H}=-21.1 \pm 0.1$ and a space density of $(2.0 \pm 0.1) \times 10^{-3} \mathrm{Mpc}^{-3}$. We show that the mass-weighted stellar ages of the PM2GC ellipticals ( $\sim 10 \mathrm{Gyr}$, but with a systematic uncertainty of \pm 2 Gyr) are in broad agreement with the look-back times to the ALESS SMGs, providing support for our simple evolutionary model.

9. We test our simple evolutionary model against intermediateand high-redshift populations of quiescent spheroidal galaxies. We find that a morphologically classified sample of ellipticals at $z \sim 0.5$ shows broad agreement in shape and number density to the "faded" ALESS SMGs at this epoch, but they are typically $\sim 0.5 \pm 0.3 \mathrm{mag}$ fainter. In addition, recent NIR spectroscopy of quiescent, red spheroids at $z=1.5-2$, the likely descendants of SMGs, indicates that their stellar populations formed at $z \sim 2.6$, consistent with the median redshift of the ALESS SMGs, $z_{\text {phot }}=2.5 \pm 0.2$.

10. Finally, we show that the X-ray-detected SMGs (Wang et al. 2013) are indistinguishable in terms of $H$-band luminosities, SFRs, and IRAC flux ratios to the ALESS SMGs brighter than $L_{H}>0.5 \times 10^{12} L_{\odot}$. We use the X-ray properties of these SMGs to estimate a median black hole mass of $M_{\mathrm{BH}} \sim\left(3_{-1}^{+3}\right) \times 10^{7} M_{\odot}$, which, combined with stellar mass estimates, indicates that the black holes in these SMGs are required to grow by approximately an order of magnitude to match the local black hole-spheroid mass relation.
We have presented the redshift distribution of a large sample of $870 \mu \mathrm{m}$ selected SMGs with precise interferometrically determined positions from ALMA. Crucially, the redshift distribution of the ALESS SMGs declines steadily from $z \sim 2.5$ and does not plateau at high redshift $(z>3)$, as has been suggested by smaller samples of millimeter-selected SMGs. Furthermore, we present a simple evolutionary scenario where SMGs undergo a period of intense star formation, before passively evolving into local elliptical galaxies. We compare the number density and "faded" $H$-band luminosity of the ALESS SMGs to local and intermediate-redshift samples of elliptical galaxies and find that both are in agreement with our simple evolutionary model. Our toy model is consistent with an evolutionary scenario where most of the stars in the majority of local luminous elliptical galaxies are formed through a single starburst SMG event at high redshift.

J.M.S. and A.L.R.D. acknowledge the support of STFC studentships (ST/J501013/1 and ST/F007299/1, respectively). A.M.S. acknowledges financial support from an STFC Advanced Fellowship (ST/H005234/1). I.R.S. acknowledges support from a Leverhulme Senior Fellowship, ERC Advanced Investigator program DUSTYGAL 321334, RS/Wolfson Merit Award, and STFC (ST/I001573/1). F.B. and A.K. acknowledge support by the Collaborative Research Council 956, sub-project A1, funded by the Deutsche Forschungsgemeinschaft (DFG). R.J.I. acknowledges support from ERC via the Advanced Grant, COSMICISM. J.W.L. acknowledges support for program number HST-GO-12866.13-A provided by NASA through a grant from the Space Telescope Science Institute, which is operated by the Association of Universities for Research in Astronomy, Incorporated, under NASA contract NAS5-26555, and the Dark Cosmology Centre, which is funded by the Danish National Research Foundation. W.N.B. acknowledges support from a Chandra X-Ray Center grant AR3-14015X and Space Telescope Science Institute grant HST-GO-12866.01-A.

The ALMA data used in this paper were obtained under program ADS/JAO.ALMA\#2011.0.00294.S. ALMA is a partnership of ESO (representing its member states), NSF (USA) and NINS (Japan), together with NRC (Canada) and NSC and ASIAA (Taiwan), in cooperation with the Republic of Chile. The Joint ALMA Observatory is operated by ESO, AUI/NRAO, and NAOJ. This publication also makes use of data acquired with the APEX under program IDs 078.F-9028(A), 079.F-9500(A), 080.A-3023(A), and 081.F-9500(A). APEX is a collaboration between the Max-Planck-Institut für Radioastronomie, the European Southern Observatory, and the Onsala Space Observatory. In this paper we make use of spectroscopic data obtained as part of the ESO/VLT large program zLESS:183.A-0666 and the VLT/XSHOOTER proposal 090.A.0927.

This research has made use of data from the HerMES project (http://hermes.sussex.ac.uk/; Oliver et al. 2012). HerMES is a Herschel Key Programme utilizing Guaranteed Time from the SPIRE instrument team, ESAC scientists, and a mission scientist.

All data used in this analysis can be obtained from the ALMA, ESO, Spitzer, and Herschel archives.

\section{APPENDIX A}

In Figure 14, we present the measured photometry and bestfitting SED for each of the ALESS SMGs. We find that the 

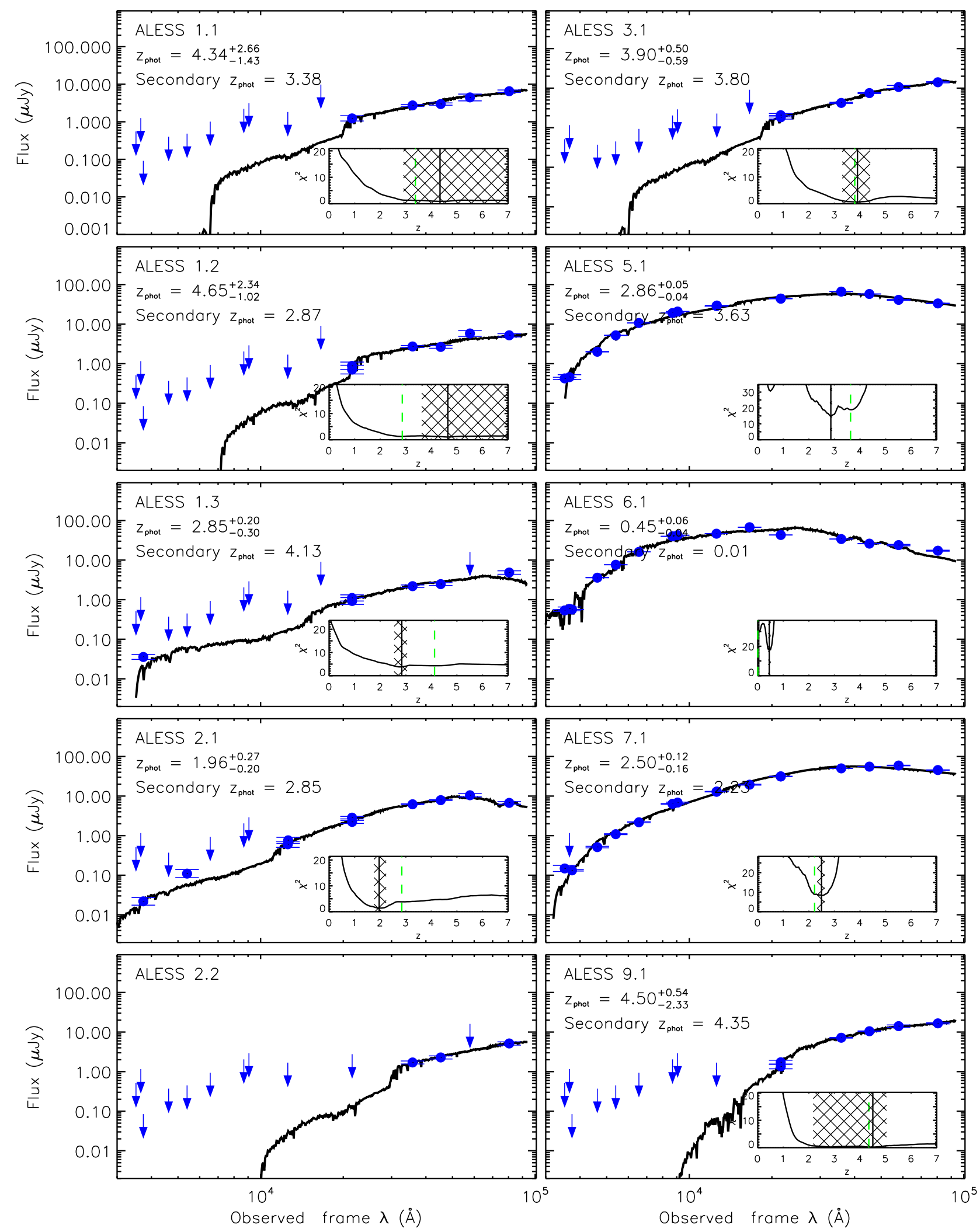

Figure 14. Photometry and best-fit spectral energy distribution for all 96 ALESS SMGs we consider in this study. Data points and errors are observed photometry, and arrows indicate $3 \sigma$ detection limits. Although we present the photometry for all sources, only redshifts derived from $\geqslant 4$ photometric detections are considered in our results. In the inset panel in each plot we show the $\chi^{2}$ distribution as a function of redshift and indicate the best-fit photometric redshift with a solid line. The hatched region shows the uncertainty on the derived redshift. Secondary redshifts are returned by HYPERZ when a secondary minimum has a $>10 \%$ probability of being true, based on the reduced $\chi^{2}$, and where appropriate these are indicated with a green dashed line.

(A color version of this figure is available in the online journal.) 

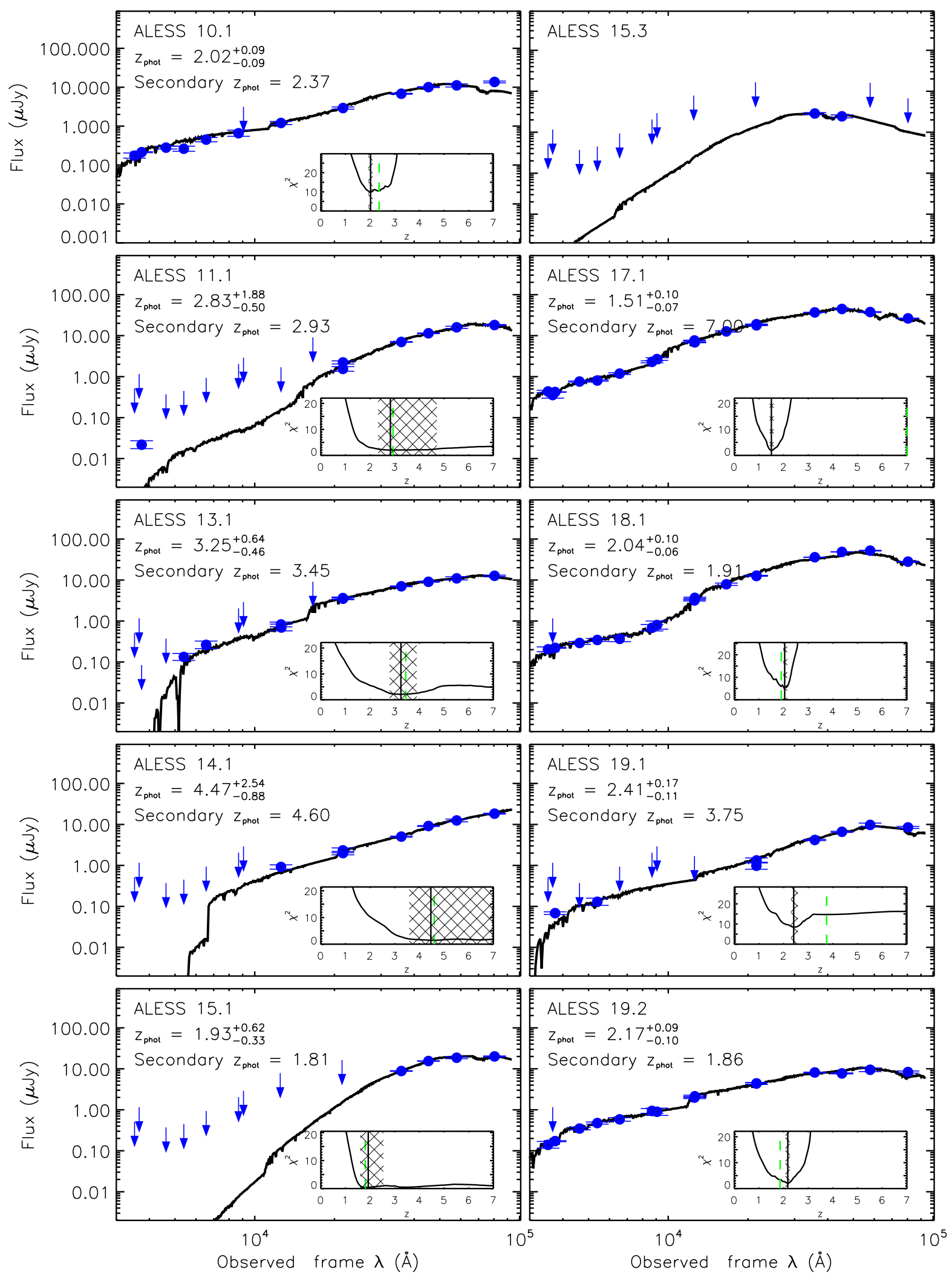

Figure 14. (Continued) 

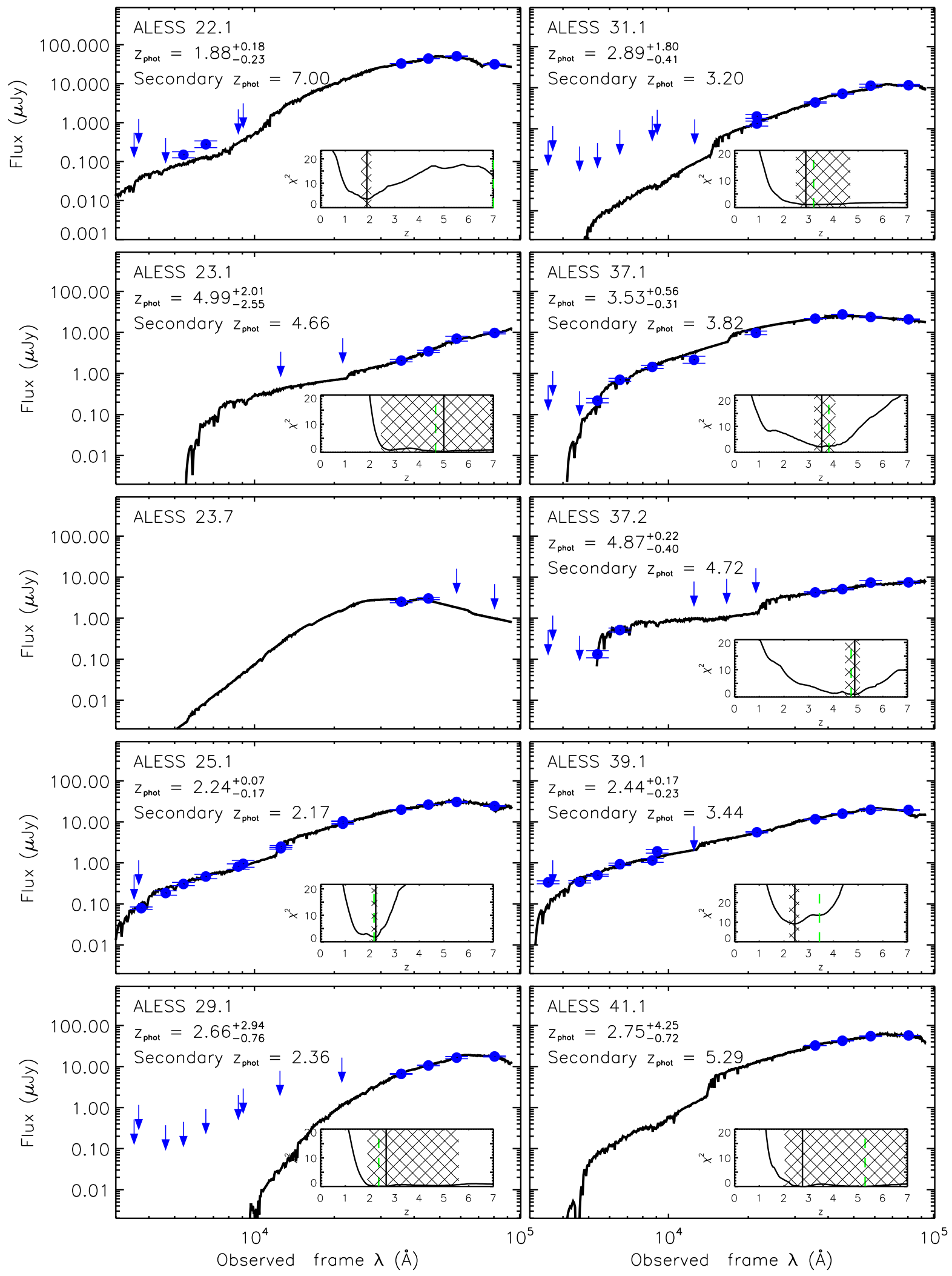

Figure 14. (Continued) 

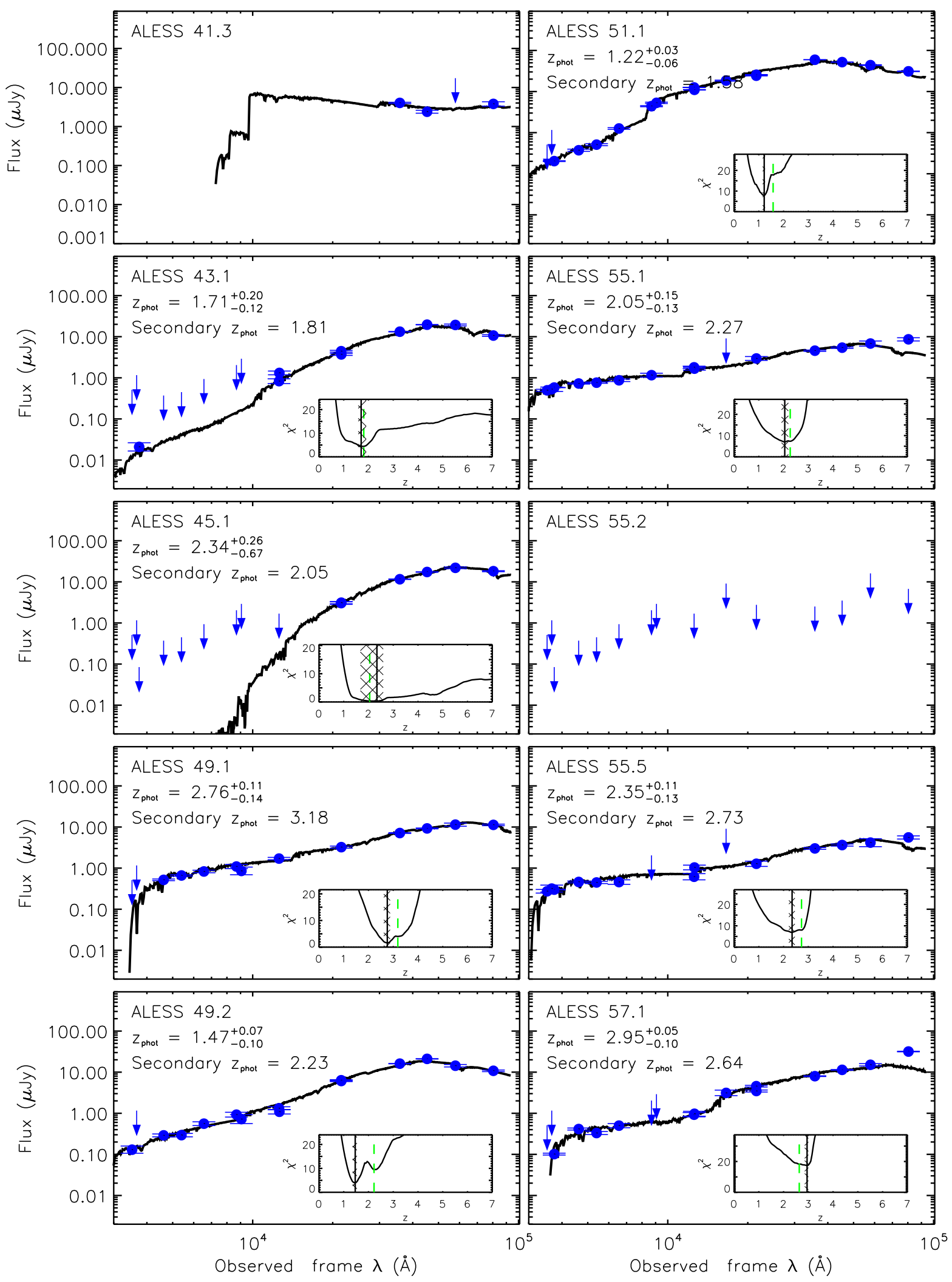

Figure 14. (Continued) 

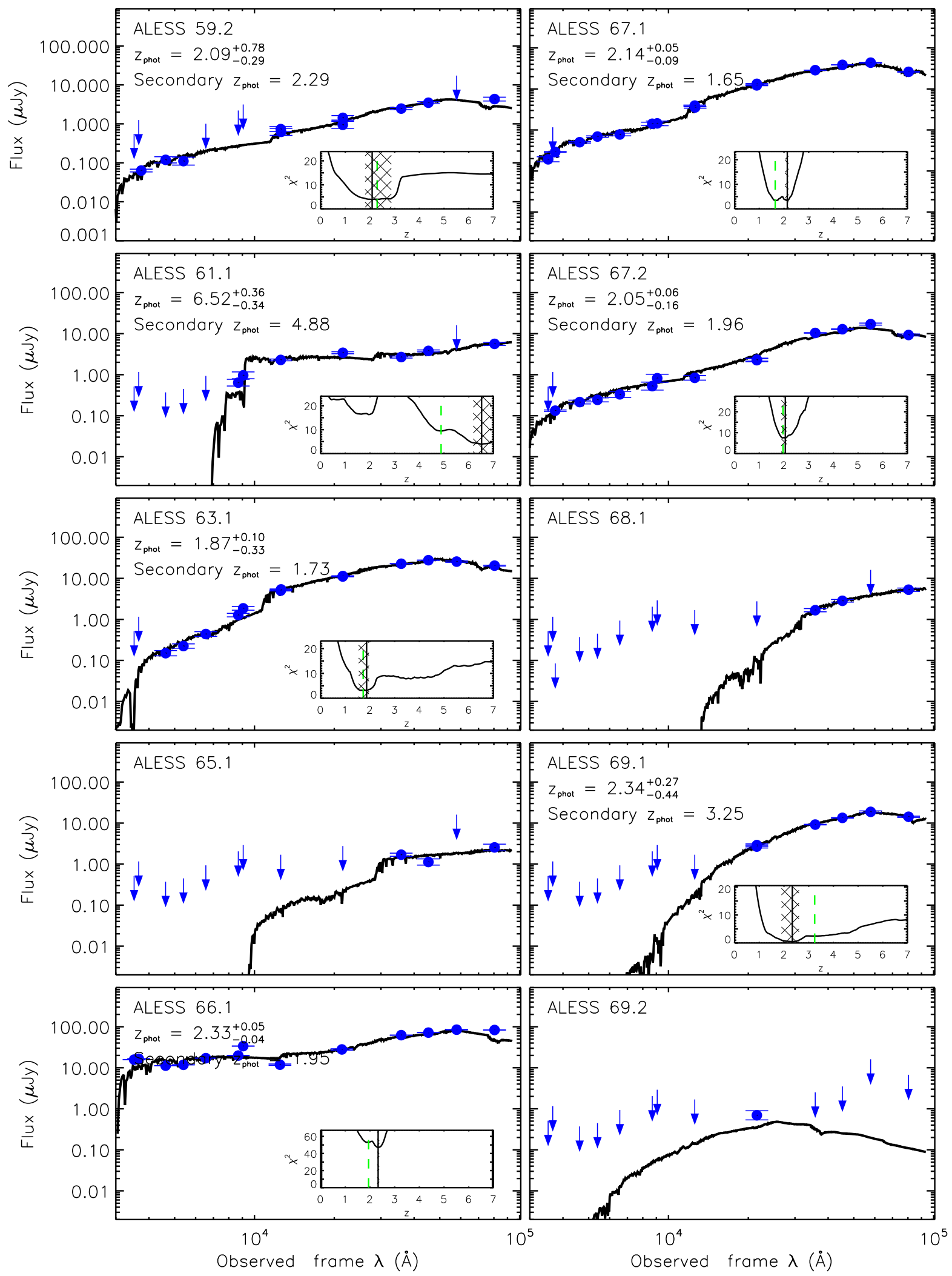

Figure 14. (Continued) 

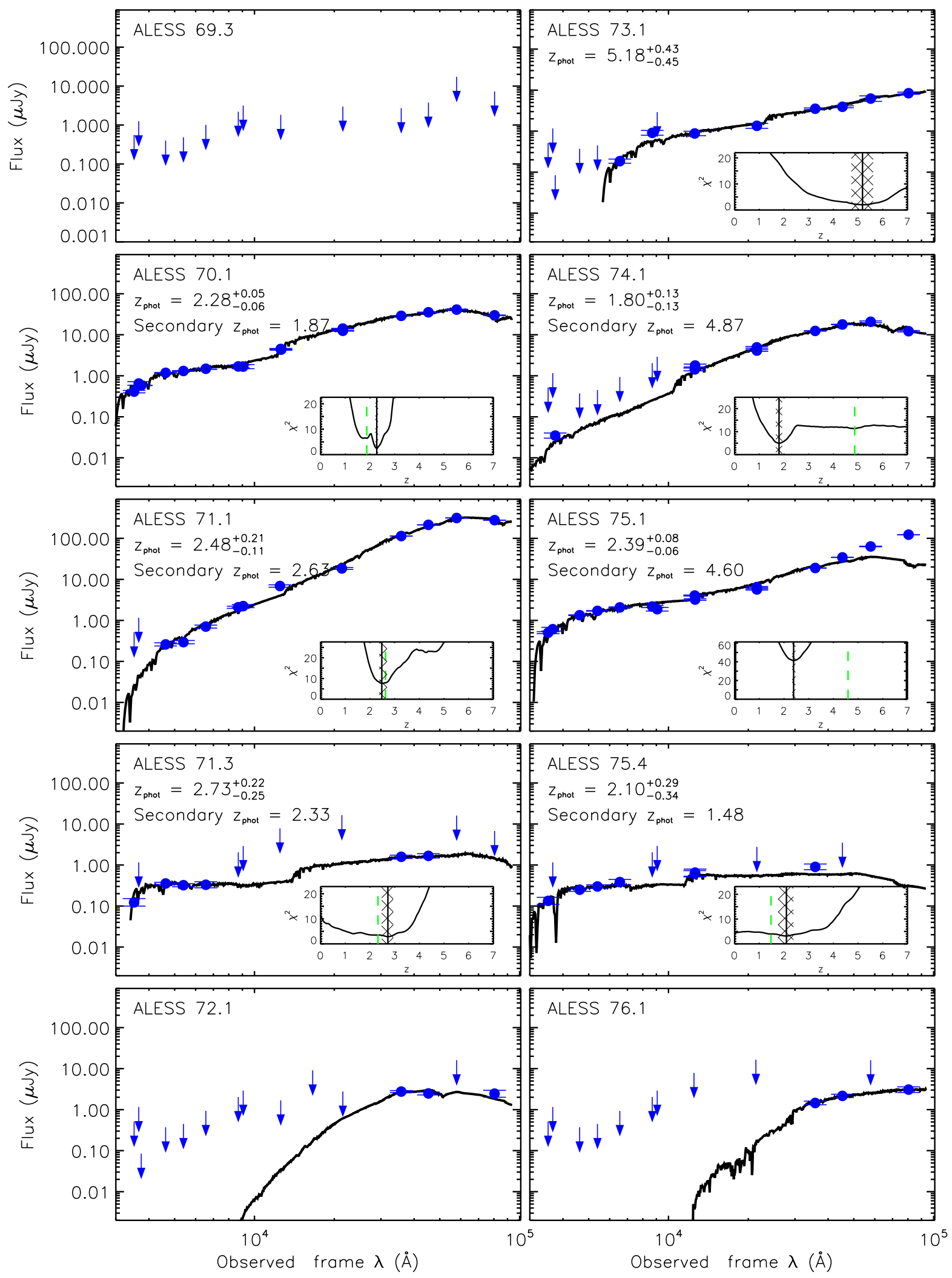

Figure 14. (Continued) 

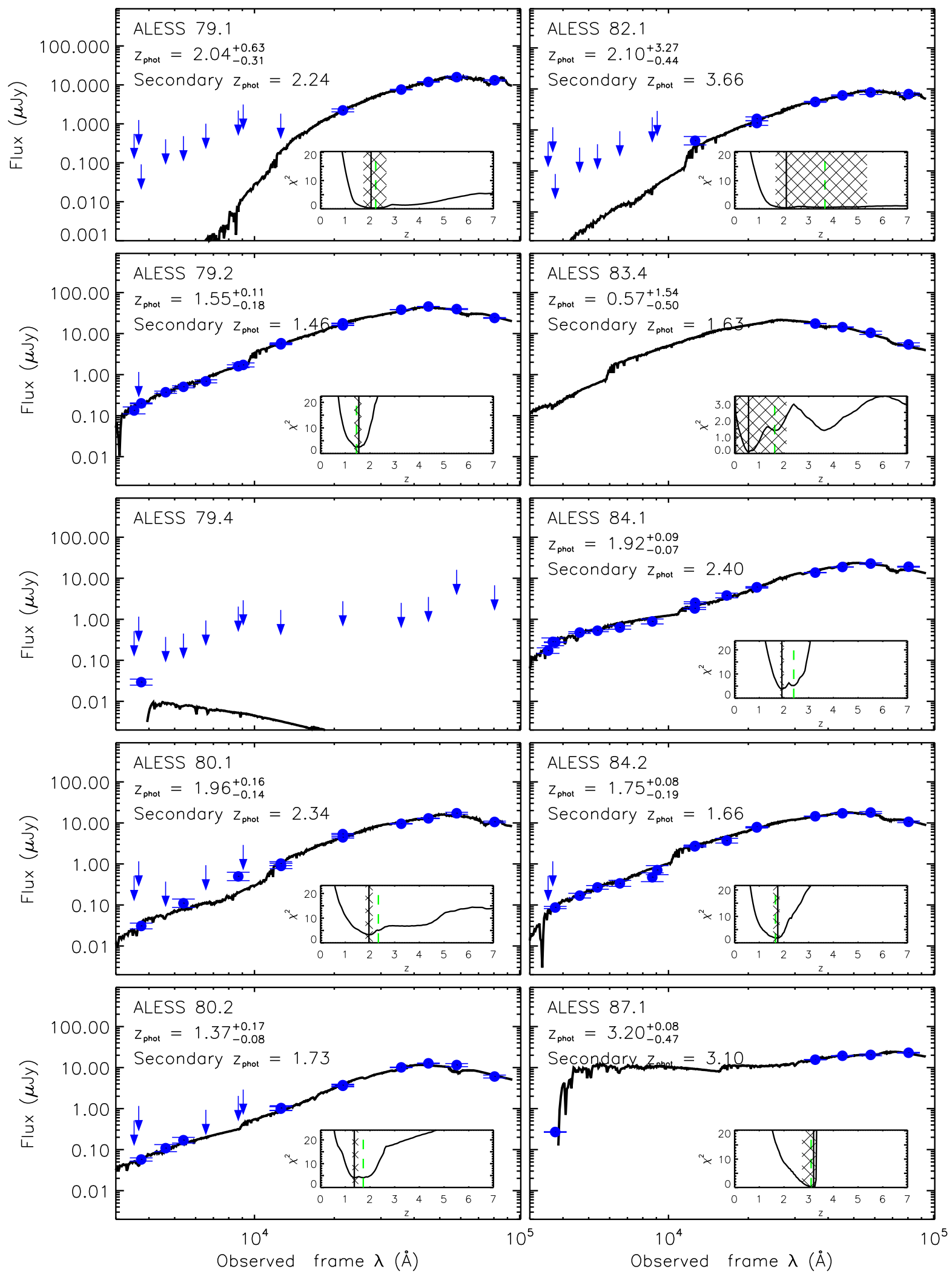

Figure 14. (Continued) 

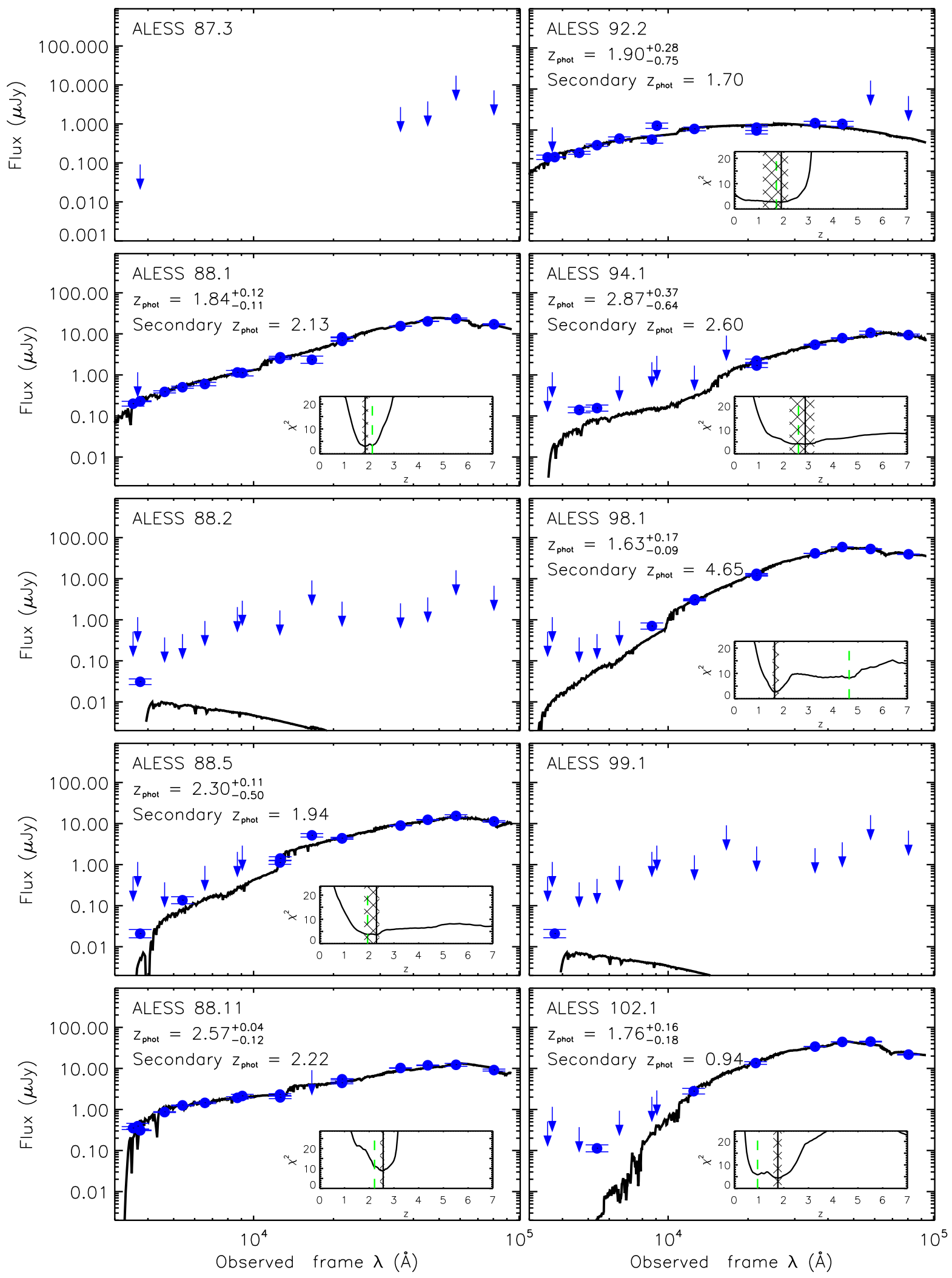

Figure 14. (Continued) 

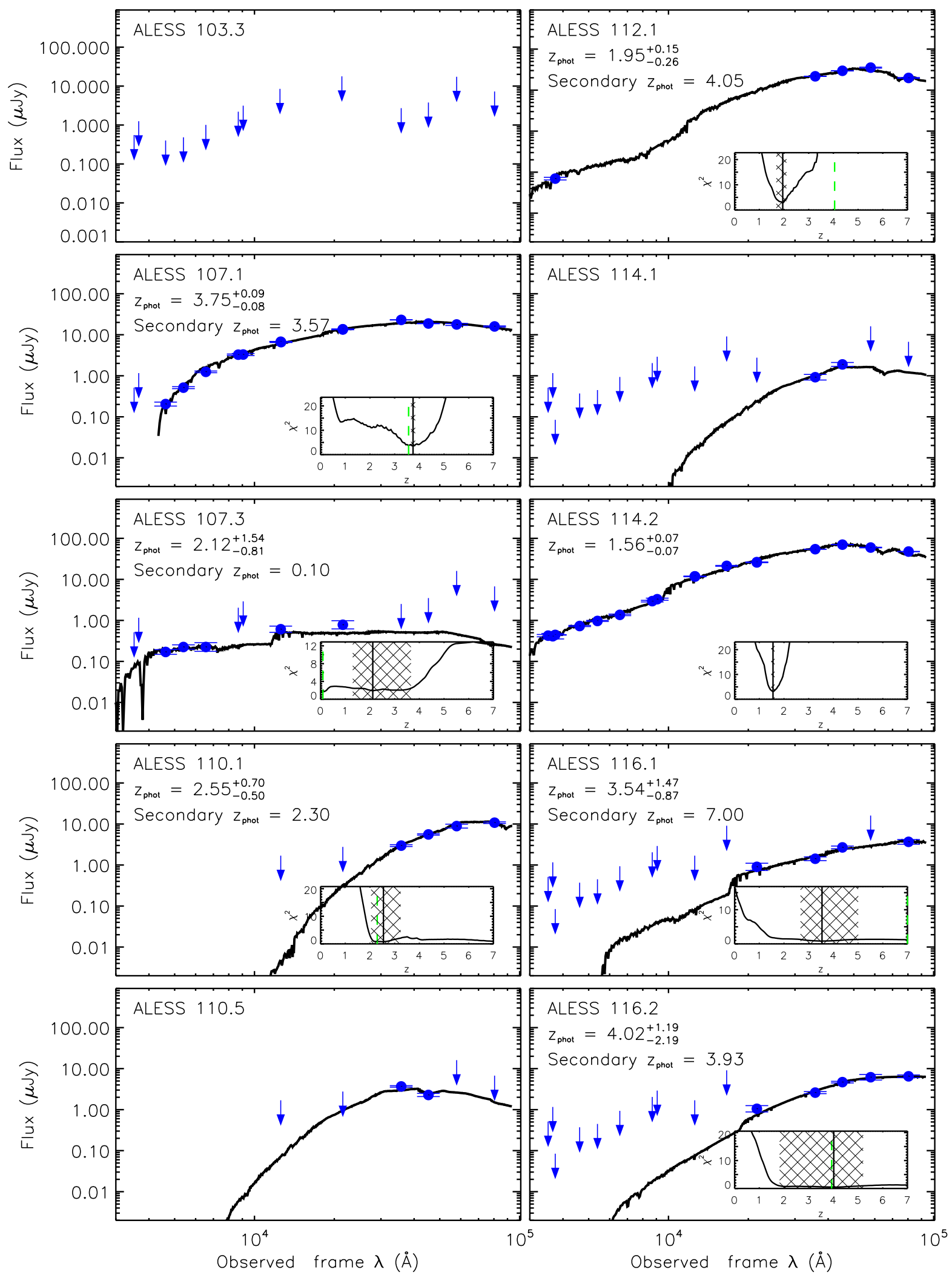

Figure 14. (Continued) 

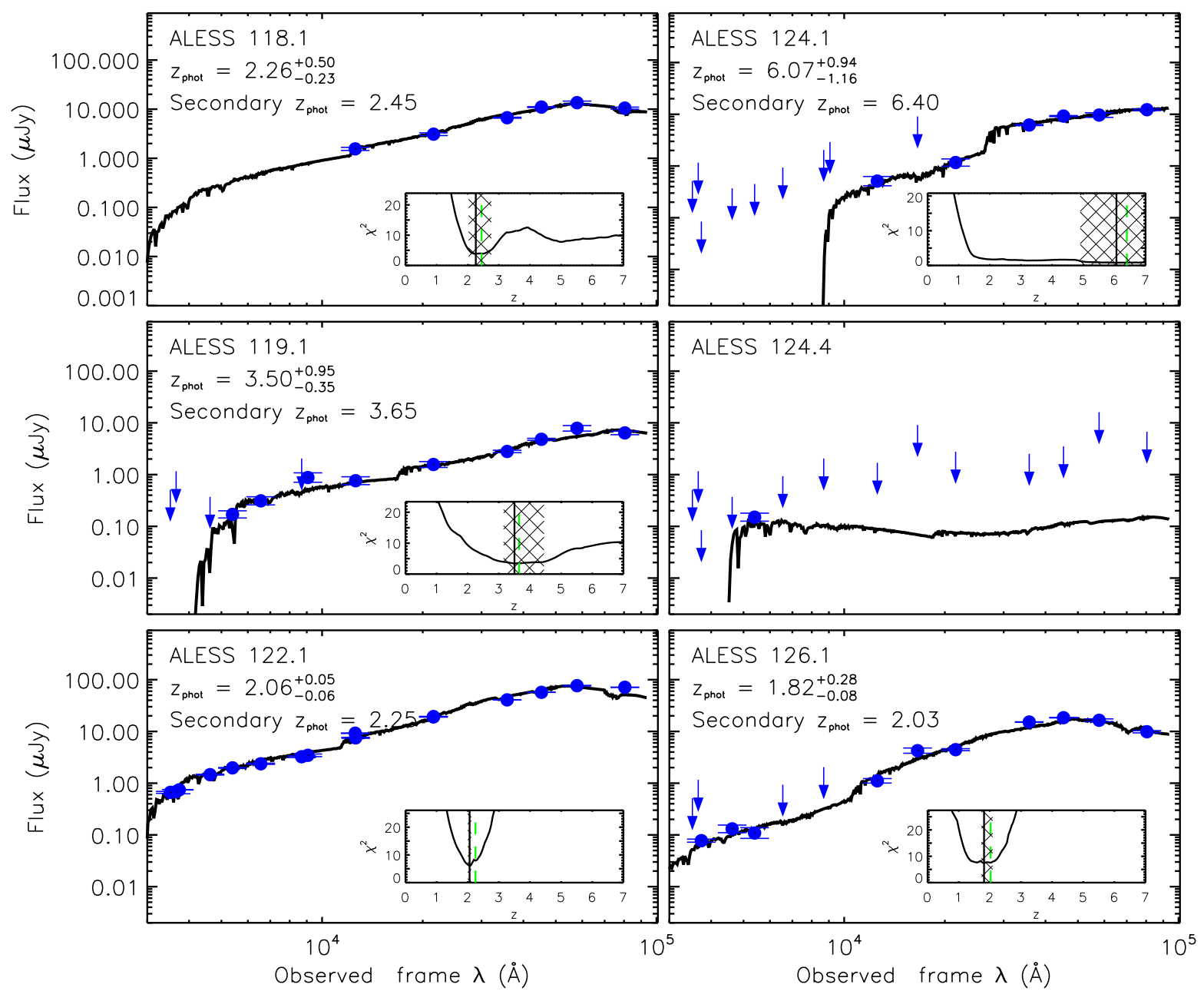

Figure 14. (Continued)

majority of our SMGs are well-fit by the best-fit SED template, and only six SMGs display evidence of an $8 \mu \mathrm{m}$ excess above the best-fit SED.

\section{APPENDIX B}

In Figure 15, we present $8^{\prime \prime} \times 8^{\prime \prime}$ optical (coadded B, I and $\mathrm{K}_{\mathrm{S}}$ ) and near-infrared (coadded 3.6, 4.5 and $8.0 \mu \mathrm{m}$ ) false color images for all 96 SMGs considered in our analysis. Crucially, our ALMA observations pinpoint the location of the $870 \mu \mathrm{m}$ emission to $<0.3^{\prime \prime}$ precision identifying the optical/ near-infrared counterpart without the requirement for indirect statistical associations.

\section{APPENDIX C}

Here we consider the SUPPLEMENTARY catalog of ALESS sources (Hodge et al. 2013). We present SED fits to the measured photometry of the (Table 4) 14 sources with $\geqslant 4$ detections (Figure 16) and provide the derived properties in Table 5. As we expect a larger fraction of spurious sources in the SUPPLEMENTARY catalog, we do not perform any analysis on the non-detections. 


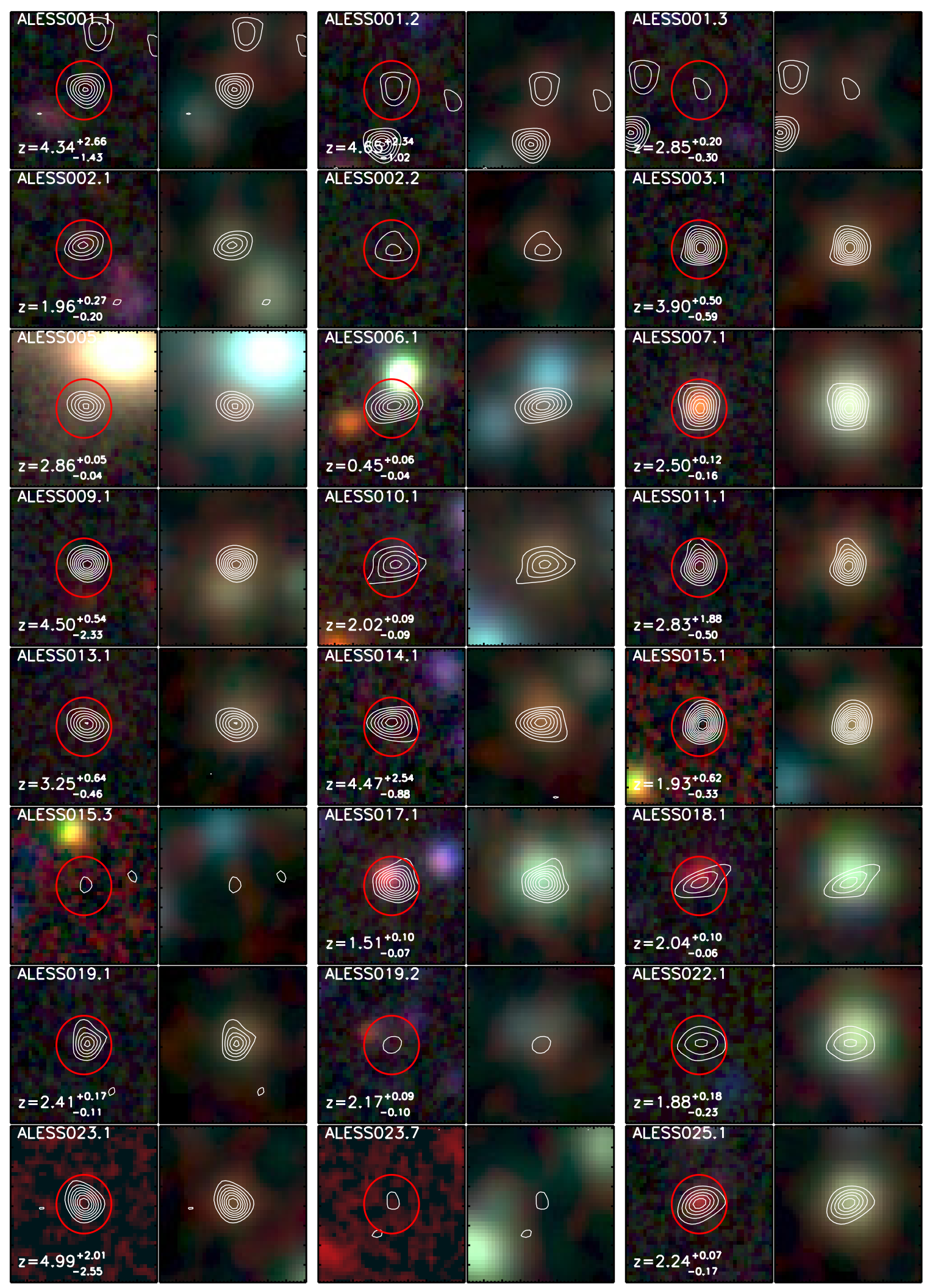

Figure 15. Here we show $8^{\prime \prime} \times 8^{\prime \prime} B I K_{s}$ (left) and $3.6 \mu \mathrm{m}, 4.5 \mu \mathrm{m}$, and $8.0 \mu \mathrm{m}$ (right) color images for each ALESS SMG. Contours indicate $870 \mu \mathrm{m}$ detections at 3 , $5,7 \ldots \times \sigma$. The ALESS SMGs are typically aligned with an NIR counterpart, and where detected they appear red in the optical $B I K_{s}$ images. A red circle represents the aperture used to measure the photometry of each SMG.

(A color version of this figure is available in the online journal.) 

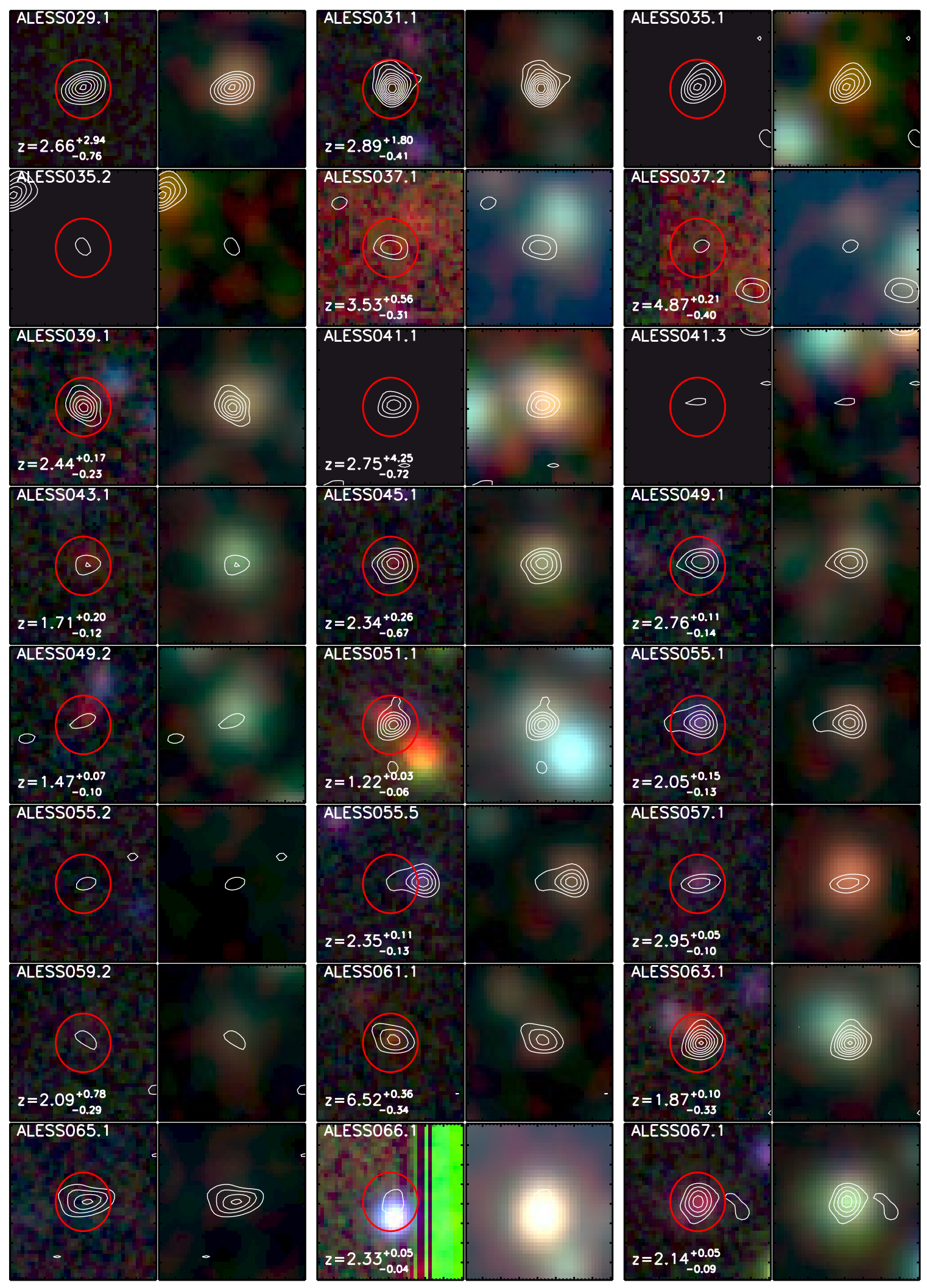

Figure 15. (Continued) 

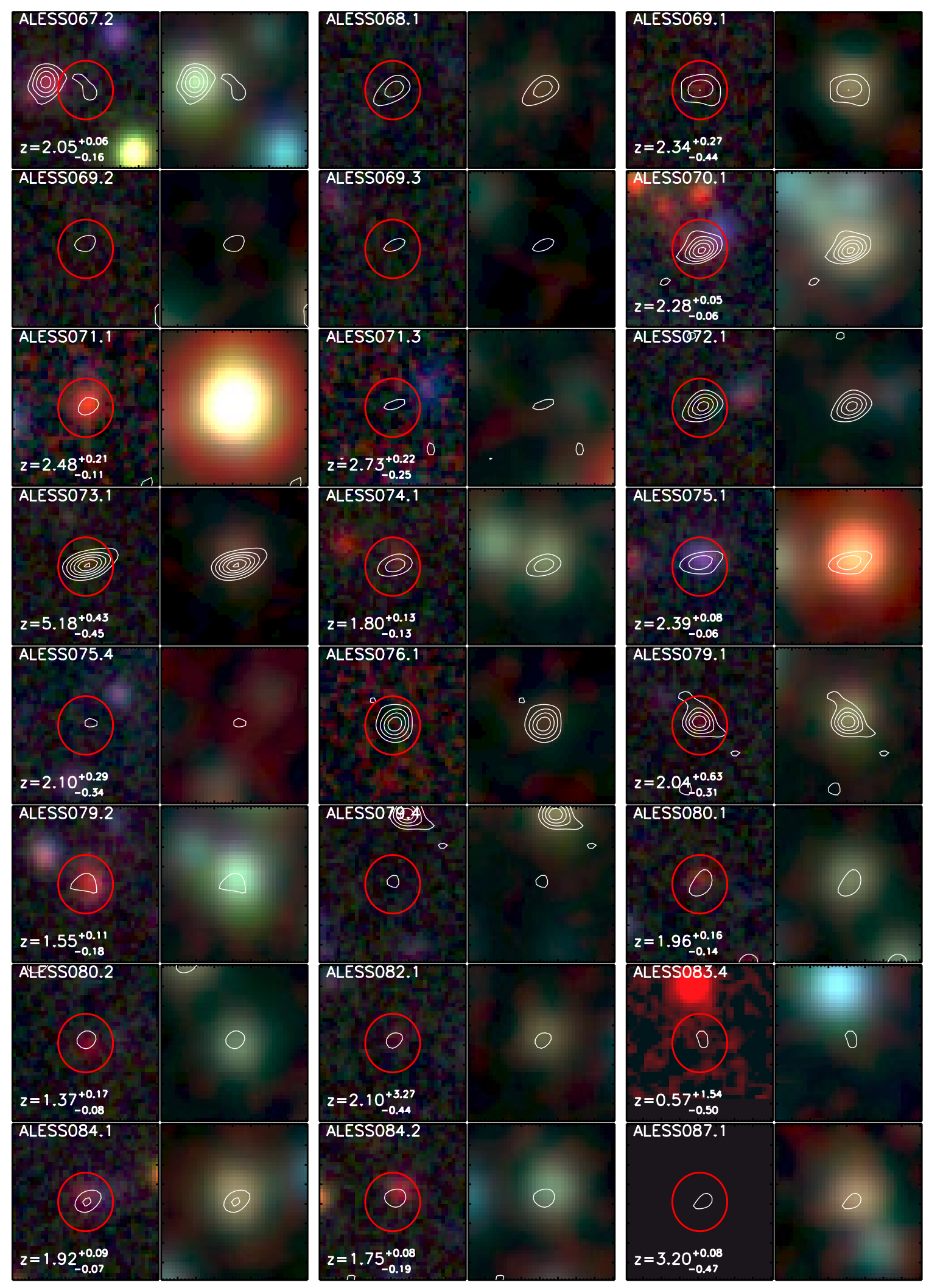

Figure 15. (Continued) 

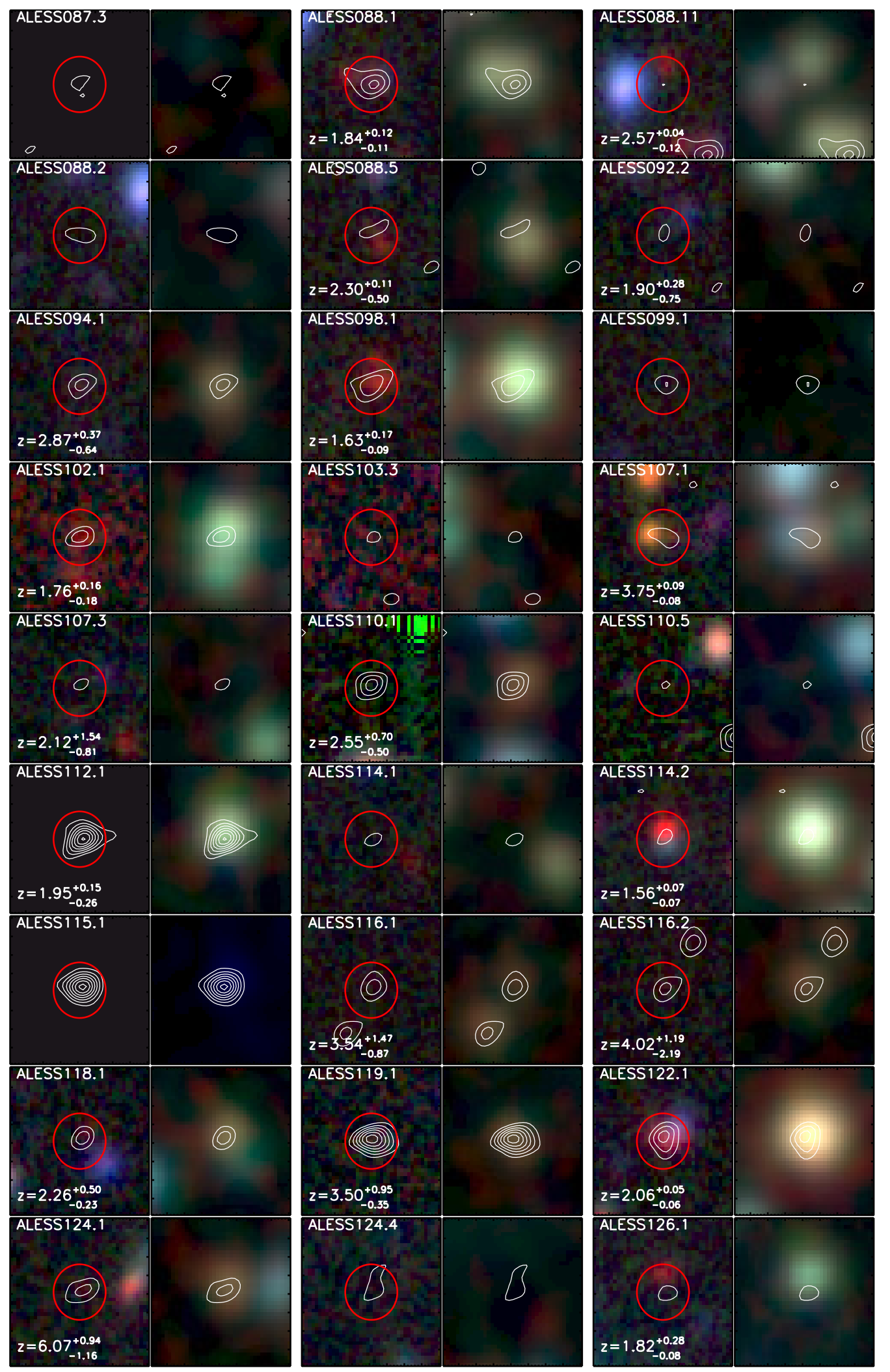

Figure 15. (Continued) 
Table 4

Photometry: Supplementary Sources

\begin{tabular}{|c|c|c|c|c|c|c|c|c|c|c|c|c|c|c|c|}
\hline ID & MUSYC $U$ & MUSYC $U_{38}$ & VIMOS $U$ & $B$ & V & $R$ & $I$ & $z$ & $J^{a}$ & $H$ & $K^{\mathrm{a}}$ & $3.6 \mu \mathrm{m}$ & $4.5 \mu \mathrm{m}$ & $5.8 \mu \mathrm{m}$ & $8.0 \mu \mathrm{m}$ \\
\hline ALESS 3.2 & $>26.18$ & $>25.29$ & $\ldots$ & $>26.53$ & $>26.32$ & $>25.53$ & $>24.68$ & $>24.29$ & $>24.88$ & & $23.39 \pm 0.12$ & $22.15 \pm 0.03$ & $21.79 \pm 0.03$ & $22.05 \pm 0.16$ & $22.27 \pm 0.08$ \\
\hline ALESS 17.2 & $\cdots$ & $>25.29$ & $25.94 \pm 0.04$ & $25.77 \pm 0.14$ & $25.71 \pm 0.16$ & $25.38 \pm 0.24$ & $>24.68$ & $>24.29$ & $>24.88$ & $>23.06$ & $>24.35$ & $24.43 \pm 0.24$ & $>24.09$ & $>22.44$ & $>23.38$ \\
\hline ALESS 17.3 & $>26.18$ & $>25.29$ & $26.04 \pm 0.04$ & $25.80 \pm 0.14$ & $26.05 \pm 0.21$ & $>25.53$ & $>24.68$ & $>24.29$ & $>24.88$ & $>23.06$ & $24.30 \pm 0.26$ & $23.60 \pm 0.12$ & $23.51 \pm 0.15$ & $>22.44$ & $>23.38$ \\
\hline ALESS 20.1 & $>26.18$ & $>25.29$ & $\ldots$ & $26.08 \pm 0.18$ & $25.49 \pm 0.13$ & $>25.53$ & $>24.68$ & $>24.29$ & $24.24 \pm 0.20^{*}$ & $>23.06$ & $22.27 \pm 0.06^{*}$ & $21.44 \pm 0.02$ & $21.10 \pm 0.02$ & $20.76 \pm 0.05$ & $21.07 \pm 0.03$ \\
\hline ALESS 34.1 & $>26.18$ & $>25.29$ & $27.37 \pm 0.14$ & $>26.53$ & $>26.32$ & $>25.53$ & $>24.68$ & $>24.29$ & $>24.88$ & $\ldots$ & $>24.35$ & $23.17 \pm 0.08$ & $23.04 \pm 0.10$ & $>22.44$ & $23.09 \pm 0.17$ \\
\hline ALESS 38.1 & $24.88 \pm 0.09$ & $24.56 \pm 0.14$ & $24.76 \pm 0.01$ & $23.89 \pm 0.03$ & $23.43 \pm 0.02$ & $23.20 \pm 0.03$ & $22.99 \pm 0.06$ & $22.93 \pm 0.08$ & $22.84 \pm 0.05$ & $\cdots$ & $21.83 \pm 0.03$ & $20.98 \pm 0.01$ & $20.61 \pm 0.01$ & $\ldots$ & $20.66 \pm 0.02$ \\
\hline ALESS 62.2 & $26.00 \pm 0.23$ & $>25.29$ & $26.08 \pm 0.04$ & $25.25 \pm 0.09$ & $24.77 \pm 0.07$ & $24.13 \pm 0.08$ & $23.21 \pm 0.08$ & $23.33 \pm 0.12$ & $22.24 \pm 0.03$ & $\cdots$ & $20.73 \pm 0.01$ & $19.61 \pm 0.00$ & $19.43 \pm 0.00$ & $19.76 \pm 0.02$ & $19.96 \pm 0.01$ \\
\hline ALESS 75.2 & $25.80 \pm 0.19$ & $>25.29$ & $\ldots$ & $23.83 \pm 0.02$ & $22.85 \pm 0.01$ & $22.08 \pm 0.01$ & $21.55 \pm 0.02$ & $21.71 \pm 0.03$ & $21.12 \pm 0.01$ & $\cdots$ & $20.67 \pm 0.01$ & $20.68 \pm 0.01$ & $20.83 \pm 0.01$ & $21.67 \pm 0.11$ & $21.84 \pm 0.06$ \\
\hline ALESS 81.1 & $\ldots$ & $\cdots$ & $\cdots$ & . & $\ldots$ & $\ldots$ & $\ldots$ & $\ldots$ & $\ldots$ & $\cdots$ & $\ldots$ & $19.79 \pm 0.00$ & $19.48 \pm 0.00$ & $19.65 \pm 0.02$ & $20.18 \pm 0.01$ \\
\hline ALESS 83.1 & $\ldots$ & $\ldots$ & $\ldots$ & $\ldots$ & $\ldots$ & $\ldots$ & $\ldots$ & $\ldots$ & $\ldots$ & $\cdots$ & $\ldots$ & $23.33 \pm 0.09$ & $22.64 \pm 0.07$ & $21.68 \pm 0.11$ & $21.79 \pm 0.05$ \\
\hline ALESS 89.1 & $25.17 \pm 0.11$ & $25.08 \pm 0.22$ & $24.93 \pm 0.02$ & $24.73 \pm 0.06$ & $24.46 \pm 0.05$ & $23.71 \pm 0.06$ & $22.82 \pm 0.05$ & $22.67 \pm 0.07$ & $22.23 \pm 0.03$ & $\cdots$ & $21.44 \pm 0.02$ & $20.84 \pm 0.01$ & $20.90 \pm 0.01$ & $21.30 \pm 0.08$ & $21.47 \pm 0.04$ \\
\hline ALESS 101.1 & $>26.18$ & $>25.29$ & $>28.14$ & $>26.53$ & $>26.32$ & $>25.53$ & $>24.68$ & $>24.29$ & & $>23.06$ & $23.08 \pm 0.09$ & $22.10 \pm 0.03$ & $21.59 \pm 0.03$ & $21.21 \pm 0.08$ & $21.15 \pm 0.03$ \\
\hline ALESS 103.2 & $>26.18$ & $>25.29$ & $\cdots$ & $25.84 \pm 0.15$ & $25.57 \pm 0.14$ & $24.91 \pm 0.16$ & $23.45 \pm 0.09$ & $23.37 \pm 0.12$ & $22.07 \pm 0.10^{* *}$ & $\cdots$ & $21.08 \pm 0.09^{* *}$ & $20.14 \pm 0.01$ & $20.24 \pm 0.01$ & $20.66 \pm 0.05$ & $21.15 \pm 0.03$ \\
\hline ALESS 106.1 & $>26.18$ & $>25.29$ & $\ldots$ & $>26.53$ & $>26.32$ & $>25.53$ & $>24.68$ & $>24.29$ & $\ldots$ & $\cdots$ & $23.71 \pm 0.16$ & $22.55 \pm 0.05$ & $22.19 \pm 0.05$ & $21.91 \pm 0.14$ & $21.45 \pm 0.04$ \\
\hline
\end{tabular}

Notes. $3 \sigma$ upper limits are presented for non-detections, and photometry is left blank where a source is not covered by available imaging.

a We measure $J$ and $K$ photometry from three imaging surveys, but quote a single value, in order of $3 \sigma$ detection limit.

Photometry measured from HAWK-I imaging.

* Photometry measured from MUSYC imaging. 

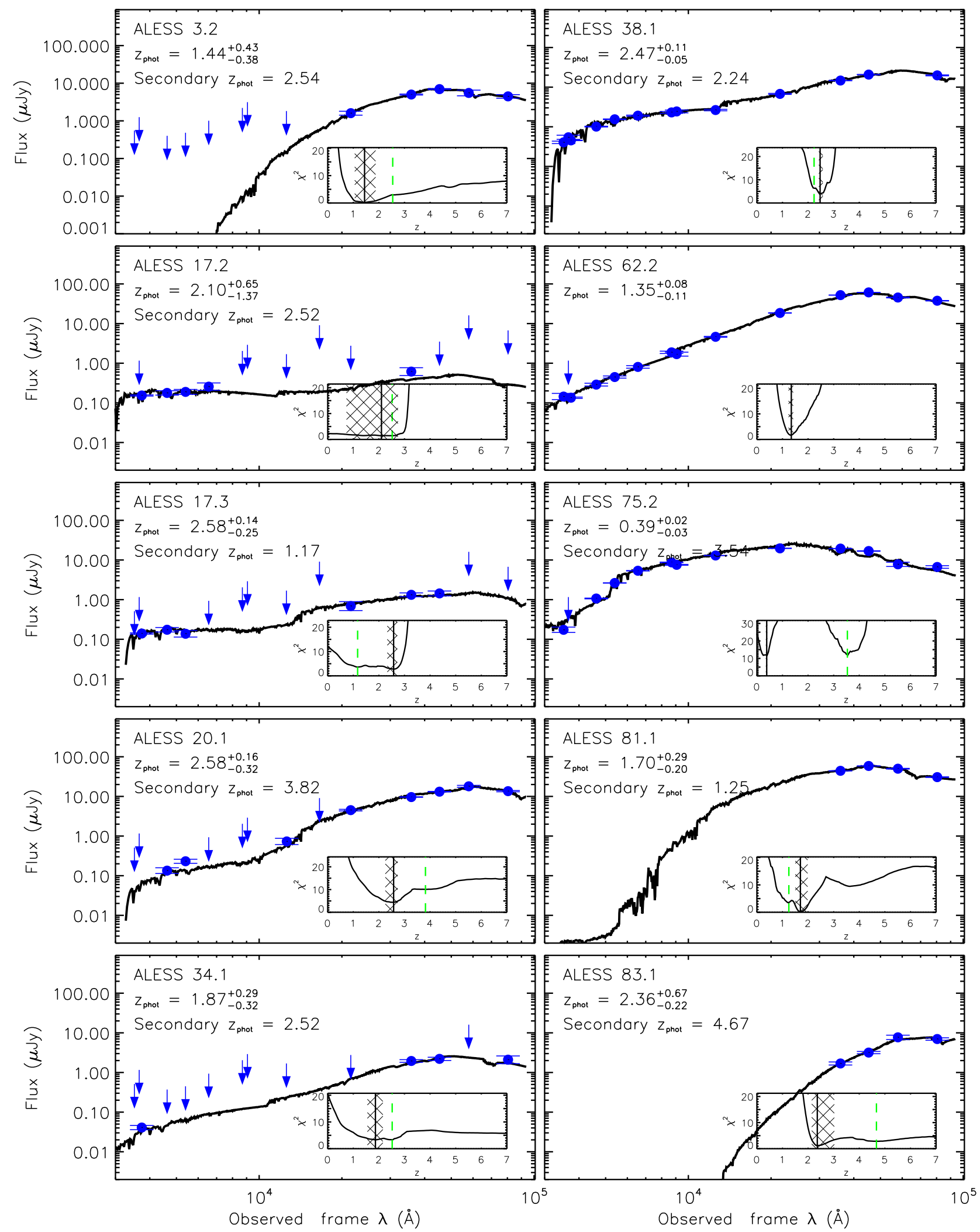

Figure 16. Photometry and best-fit spectral energy distribution for 14 SMGs from the ALESS suPPLEMENTARY catalog (Hodge et al. 2013). Data points and errors are observed photometry, and arrows indicate $3 \sigma$ detection limits. In the inset panel in each plot we show the $\chi^{2}$ distribution as a function of redshift, indicating the best-fit photometric redshift with a solid line, and where appropriate the secondary solution with a green dashed line. The hatched region shows the uncertainty on the derived redshift.

(A color version of this figure is available in the online journal.) 

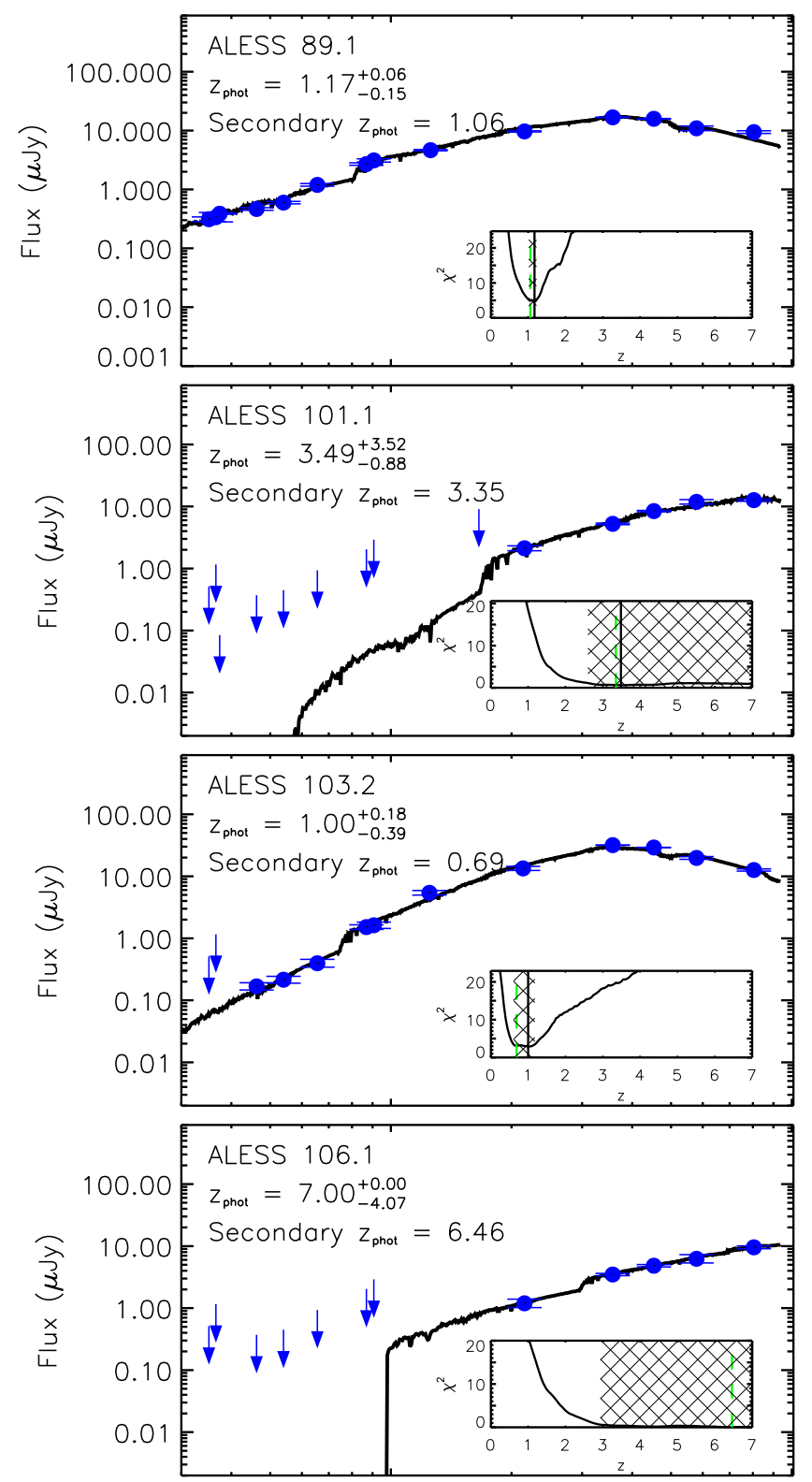

Figure 16. (Continued)

Table 5

Derived Properties: Supplementary Sources

\begin{tabular}{|c|c|c|c|c|c|c|c|}
\hline ID & $\begin{array}{l}\text { R.A. } \\
\text { (J2000) }\end{array}$ & $\begin{array}{c}\text { Decl. } \\
(\mathrm{J} 2000)\end{array}$ & $z_{\text {phot }}$ & $\chi_{\text {red }}^{2}$ & $\begin{array}{c}\text { Filters } \\
\text { (Det [Obs]) }\end{array}$ & $\begin{array}{c}M_{H} \\
(\mathrm{AB})\end{array}$ & $\begin{array}{c}M / L_{H} \\
\left(M_{\odot} L_{\odot}^{-1}\right)\end{array}$ \\
\hline ALESS 003.2 & 03:33:22.19 & $-27: 55: 20.9$ & $1.44_{-0.38}^{+0.43}$ & 0.23 & 5 [13] & -22.34 & 0.36 \\
\hline ALESS 017.2 & 03:32:08.26 & $-27: 51: 19.7$ & $2.10_{-1.37}^{+0.65}$ & 1.43 & $5[14]$ & -20.76 & 0.15 \\
\hline ALESS 017.3 & 03:32:07.37 & $-27: 51: 33.9$ & $2.58_{-0.25}^{+0.14}$ & 2.79 & $6[15]$ & -21.97 & 0.15 \\
\hline ALESS 020.1 & 03:33:16.76 & $-28: 00: 16.0$ & $2.58_{-0.32}^{+0.16}$ & 4.38 & $8[14]$ & -24.74 & 0.18 \\
\hline ALESS 034.1 & 03:32:17.96 & $-27: 52: 33.3$ & $1.87_{-0.32}^{+0.29}$ & 3.32 & $4[14]$ & -21.70 & 0.15 \\
\hline ALESS 038.1 & 03:33:10.84 & $-27: 56: 40.2$ & $2.47_{-0.05}^{+0.11}$ & 3.79 & 13 [13] & -24.88 & 0.05 \\
\hline ALESS 062.2 & $03: 32: 36.58$ & $-27: 34: 53.8$ & $1.35_{-0.11}^{+0.08}$ & 1.68 & $13[14]$ & -24.59 & 0.05 \\
\hline ALESS 075.2 & $03: 31: 27.67$ & $-27: 55: 59.2$ & $0.39_{-0.03}^{+0.02}$ & 11.44 & 12 [13] & -20.84 & 0.18 \\
\hline ALESS 081.1 & $03: 31: 27.55$ & $-27: 44: 39.6$ & $1.70_{-0.20}^{+0.29}$ & 0.23 & $4[4]$ & -25.04 & 0.36 \\
\hline ALESS 083.1 & 03:33:09.42 & $-28: 05: 30.6$ & $2.36_{-0.22}^{+0.67}$ & 1.22 & $4[4]$ & -23.59 & 0.36 \\
\hline ALESS 089.1 & 03:32:48.69 & $-28: 00: 21.9$ & $1.17_{-0.15}^{+0.06}$ & 4.64 & $14[14]$ & -22.88 & 0.17 \\
\hline ALESS 101.1 & 03:31:51.60 & $-27: 45: 53.0$ & $3.49_{-0.88}^{+3.52}$ & 0.59 & $5[14]$ & -25.15 & 0.18 \\
\hline ALESS 103.2 & $03: 33: 25.82$ & $-27: 34: 09.9$ & $1.00_{-0.39}^{+0.18}$ & 2.91 & $11[13]$ & -23.24 & 0.08 \\
\hline ALESS 106.1 & $03: 31: 39.64$ & $-27: 56: 39.2$ & $7.00_{-4.07}^{+0.00}$ & 0.02 & $5[12]$ & -26.31 & 0.11 \\
\hline
\end{tabular}




\section{REFERENCES}

Alaghband-Zadeh, S., Chapman, S. C., Swinbank, A. M., et al. 2012, MNRAS, 424, 2232

Alexander, D. M., Brandt, W. N., Smail, I., et al. 2008, AJ, 135, 1968

Alexander, D. M., \& Hickox, R. C. 2012, NewAR, 56, 93

Alexander, D. M., Smail, I., Bauer, F. E., et al. 2005, Natur, 434, 738

Aretxaga, I., Hughes, D. H., Coppin, K., et al. 2007, MNRAS, 379, 1571

Balestra, I., Mainieri, V., Popesso, P., et al. 2010, A\&A, 512, A12

Barger, A. J., Cowie, L. L., Sanders, D. B., et al. 1998, Natur, 394, 248

Barger, A. J., Wang, W.-H., Cowie, L. L., et al. 2012, ApJ, 761, 89

Bedregal, A. G., Scarlata, C., Henry, A. L., et al. 2013, ApJ, 778, 126

Bell, E. F., McIntosh, D. H., Katz, N., \& Weinberg, M. D. 2003, ApJS, 149,289

Bertin, E., \& Arnouts, S. 1996, A\&AS, 117, 393

Bertoldi, F., Carilli, C., Aravena, M., et al. 2007, ApJS, 172, 132

Biggs, A. D., \& Ivison, R. J. 2008, MNRAS, 385, 893

Biggs, A. D., Ivison, R. J., Ibar, E., et al. 2011, MNRAS, 413, 2314

Blain, A. W., Chapman, S. C., Smail, I., \& Ivison, R. 2004, ApJ, 611, 725

Blain, A. W., Smail, I., Ivison, R. J., \& Kneib, J.-P. 1999, MNRAS, 302, 632

Blain, A. W., Smail, I., Ivison, R. J., Kneib, J.-P., \& Frayer, D. T. 2002, PhR, 369,111

Blanton, M. R., Hogg, D. W., Bahcall, N. A., et al. 2003, ApJ, 594, 186

Bolzonella, M., Miralles, J.-M., \& Pelló, R. 2000, A\&A, 363, 476

Bonzini, M., Mainieri, V., Padovani, P., et al. 2012, ApJS, 203, 15

Bothwell, M. S., Smail, I., Chapman, S. C., et al. 2013, MNRAS, 429, 3047

Bower, R. G., Lucey, J. R., \& Ellis, R. S. 1992, MNRAS, 254, 601

Brammer, G. B., van Dokkum, P. G., \& Coppi, P. 2008, ApJ, 686, 1503

Bruzual, G., \& Charlot, S. 2003, MNRAS, 344, 1000

Bundy, K., Ellis, R. S., \& Conselice, C. J. 2005, ApJ, 625, 621

Bunker, A. J., Stanway, E. R., Ellis, R. S., McMahon, R. G., \& McCarthy, P. J. 2003, MNRAS, 342, L47

Calvi, R., Poggianti, B. M., Fasano, G., \& Vulcani, B. 2012, MNRAS, 419, L14

Calvi, R., Poggianti, B. M., \& Vulcani, B. 2011, MNRAS, 416, 727

Calvi, R., Poggianti, B. M., Vulcani, B., \& Fasano, G. 2013, MNRAS, 432, 3141

Calzetti, D., Armus, L., Bohlin, R. C., et al. 2000, ApJ, 533, 682

Carilli, C. L., Daddi, E., Riechers, D., et al. 2010, ApJ, 714, 1407

Casey, C. M., Chapman, S. C., Smail, I., et al. 2011, MNRAS, 411, 2739

Casey, C. M., Chen, C.-C., Cowie, L. L., et al. 2013, MNRAS, 436, 1919

Chapin, E. L., Pope, A., Scott, D., et al. 2009, MNRAS, 398, 1793

Chapman, S. C., Blain, A. W., Smail, I., \& Ivison, R. J. 2005, ApJ, 622, 772

Chary, R., \& Elbaz, D. 2001, ApJ, 556, 562

Cimatti, A., Mignoli, M., Daddi, E., et al. 2002, A\&A, 392, 395

Cooper, M. C., Yan, R., Dickinson, M., et al. 2012, MNRAS, 425, 2116

Coppin, K., Chapin, E. L., Mortier, A. M. J., et al. 2006, MNRAS, 372, 1621

Coppin, K. E. K., Danielson, A. L. R., Geach, J. E., et al. 2012, MNRAS, 427, 520

Coppin, K. E. K., Smail, I., Alexander, D. M., et al. 2009, MNRAS, 395,1905

Coppin, K. E. K., Swinbank, A. M., Neri, R., et al. 2008, MNRAS, 389, 45

Cristiani, S., Appenzeller, I., Arnouts, S., et al. 2000, A\&A, 359, 489

Croom, S. M., Warren, S. J., \& Glazebrook, K. 2001, MNRAS, 328, 150

Daddi, E., Renzini, A., Pirzkal, N., et al. 2005, ApJ, 626, 680

Dahlen, T., Mobasher, B., Faber, S. M., et al. 2013, ApJ, 775, 93

Damen, M., Labbé, I., van Dokkum, P. G., et al. 2011, ApJ, 727, 1

Danielson, A. L. R., Swinbank, A. M., Smail, I., et al. 2011, MNRAS, 410, 1687

Di Matteo, T., Springel, V., \& Hernquist, L. 2005, Natur, 433, 604

Doherty, M., Bunker, A. J., Ellis, R. S., \& McCarthy, P. J. 2005, MNRAS, 361,525

Dole, H., Le Floc'h, E., Pérez-González, P. G., et al. 2004, ApJS, 154, 87

Eales, S., Lilly, S., Gear, W., et al. 1999, ApJ, 515, 518

Engel, H., Tacconi, L. J., Davies, R. I., et al. 2010, ApJ, 724, 233

Fasano, G., Vanzella, E., Dressler, A., et al. 2012, MNRAS, 420, 926

Gawiser, E., van Dokkum, P. G., Herrera, D., et al. 2006, ApJS, 162, 1

Genzel, R., Baker, A. J., Tacconi, L. J., et al. 2003, ApJ, 584, 633

Granato, G. L., De Zotti, G., Silva, L., Bressan, A., \& Danese, L. 2004, ApJ, 600,580

Greve, T. R., Bertoldi, F., Smail, I., et al. 2005, MNRAS, 359, 1165

Griffin, M. J., Abergel, A., Abreu, A., et al. 2010, A\&A, 518, L3

Hainline, L. J., Blain, A. W., Smail, I., et al. 2009, ApJ, 699, 1610

Hainline, L. J., Blain, A. W., Smail, I., et al. 2011, ApJ, 740, 96

Häring, N., \& Rix, H.-W. 2004, ApJL, 604, L89

Henriques, B., Maraston, C., Monaco, P., et al. 2011, MNRAS, 415, 3571
Hezaveh, Y. D., Marrone, D. P., \& Holder, G. P. 2012, ApJ, 761, 20

Hickox, R. C., Wardlow, J. L., Smail, I., et al. 2012, MNRAS, 421, 284

Hodge, J. A., Carilli, C. L., Walter, F., et al. 2012, ApJ, 760, 11

Hodge, J. A., Karim, A., Smail, I., et al. 2013, ApJ, 768, 91

Hopkins, P. F., Hernquist, L., Cox, T. J., et al. 2006, ApJS, 163, 1

Hsieh, B.-C., Wang, W.-H., Hsieh, C.-C., et al. 2012, ApJS, 203, 23

Hughes, D. H., Serjeant, S., Dunlop, J., et al. 1998, Natur, 394, 241

Ivison, R. J., Greve, T. R., Dunlop, J. S., et al. 2007, MNRAS, 380, 199

Ivison, R. J., Greve, T. R., Serjeant, S., et al. 2004, ApJS, 154, 124

Ivison, R. J., Greve, T. R., Smail, I., et al. 2002, MNRAS, 337, 1

Ivison, R. J., Smail, I., Amblard, A., et al. 2012, MNRAS, 425, 1320

Ivison, R. J., Smail, I., Barger, A. J., et al. 2000, MNRAS, 315, 209

Ivison, R. J., Smail, I., Le Borgne, J.-F., et al. 1998, MNRAS, 298, 583

Ivison, R. J., Swinbank, A. M., Swinyard, B., et al. 2010, A\&A, 518, L35

Karim, A., Swinbank, A. M., Hodge, J. A., et al. 2013, MNRAS, 432, 2

Kelly, B. C., Vestergaard, M., Fan, X., et al. 2010, ApJ, 719, 1315

Kennicutt, R. C. 1998, ARA\&A, 36, 189

Kriek, M., van Dokkum, P. G., Franx, M., et al. 2008, ApJ, 677, 219

Lawrence, A., Warren, S. J., Almaini, O., et al. 2007, MNRAS, 379, 1599

Lawrence, A., Warren, S. J., Almaini, O., et al. 2012, yCat, 2314, 0

Le Fèvre, O., Vettolani, G., Paltani, S., et al. 2004, A\&A, 428, 1043

LeFloc'h, E., Aussel, H., Ilbert, O., et al. 2009, ApJ, 703, 222

Leitherer, C., Ortiz Otálvaro, P. A., Bresolin, F., et al. 2010, ApJS, 189, 309

Leitherer, C., Schaerer, D., Goldader, J. D., et al. 1999, ApJS, 123, 3

Lilly, S. J., Eales, S. A., Gear, W. K. P., et al. 1999, ApJ, 518, 641

Lindner, R. R., Baker, A. J., Omont, A., et al. 2011, ApJ, 737, 83

Luo, B., Brandt, W. N., Xue, Y. Q., et al. 2010, ApJS, 187, 560

Magnelli, B., Lutz, D., Santini, P., et al. 2012, A\&A, 539, A155

Martini, P., \& Weinberg, D. H. 2001, ApJ, 547, 12

McLure, R. J., \& Dunlop, J. S. 2004, MNRAS, 352, 1390

Menéndez-Delmestre, K., Blain, A. W., Swinbank, M., et al. 2013, ApJ, 767,151

Michałowski, M. J., Dunlop, J. S., Cirasuolo, M., et al. 2012, A\&A, 541, A85

Michałowski, M., Hjorth, J., \& Watson, D. 2010, A\&A, 514, A67

Mignoli, M., Cimatti, A., Zamorani, G., et al. 2005, A\&A, 437, 883

Miller, N. A., Bonzini, M., Fomalont, E. B., et al. 2013, ApJS, 205, 13

Miller, N. A., Fomalont, E. B., Kellermann, K. I., et al. 2008, ApJS, 179,114

Nelan, J. E., Smith, R. J., Hudson, M. J., et al. 2005, ApJ, 632, 137

Nonino, M., Dickinson, M., Rosati, P., et al. 2009, ApJS, 183, 244

Oliver, S. J., Bock, J., Altieri, B., et al. 2012, MNRAS, 424, 1614

Pilbratt, G. L., Riedinger, J. R., Passvogel, T., et al. 2010, A\&A, 518, L1

Poggianti, B. M., Calvi, R., Bindoni, D., et al. 2013, ApJ, 762, 77

Popesso, P., Dickinson, M., Nonino, M., et al. 2009, A\&A, 494, 443

Ravikumar, C. D., Puech, M., Flores, H., et al. 2007, A\&A, 465, 1099

Riechers, D. A., Capak, P. L., Carilli, C. L., et al. 2010, ApJL, 720, L131

Sandage, A., \& Visvanathan, N. 1978, ApJ, 225, 742

Sanders, D. B., Soifer, B. T., Elias, J. H., Neugebauer, G., \& Matthews, K. 1988, ApJL, 328, L35

Silverman, J. D., Mainieri, V., Salvato, M., et al. 2010, ApJS, 191, 124

Simpson, J. M., Smail, I., Swinbank, A. M., et al. 2012, MNRAS, 426, 3201

Smail, I., Ivison, R. J., \& Blain, A. W. 1997, ApJL, 490, L5

Smail, I., Ivison, R. J., Blain, A. W., \& Kneib, J.-P. 2002, MNRAS, 331, 495

Smolčić, V., Aravena, M., Navarrete, F., et al. 2012, A\&A, 548, A4

Stanway, E. R., Bunker, A. J., McMahon, R. G., et al. 2004, ApJ, 607, 704

Strolger, L.-G., Riess, A. G., Dahlen, T., et al. 2004, ApJ, 613, 200

Surace, J. A., Shupe, D. L., Fang, F., et al. 2005, http://swire.ipac.caltech.edu/ swire/astronomers/publications

Swinbank, A. M., Chapman, S. C., Smail, I., et al. 2006, MNRAS, 371, 465

Swinbank, A. M., Karim, A., Smail, I., et al. 2012, MNRAS, 427, 1066

Swinbank, A. M., Mikles, V. J., Mainieri, V., et al. 2014, MNRAS, 438, 1267

Swinbank, A. M., Smail, I., Chapman, S. C., et al. 2004, ApJ, 617, 64

Swinbank, A. M., Smail, I., Chapman, S. C., et al. 2010a, MNRAS, 405,234

Swinbank, A. M., Smail, I., Longmore, S., et al. 2010b, Natur, 464, 733

Szokoly, G. P., Bergeron, J., Hasinger, G., et al. 2004, ApJS, 155, 271

Tacconi, L. J., Genzel, R., Smail, I., et al. 2008, ApJ, 680, 246

Taylor, E. N., Franx, M., van Dokkum, P. G., et al. 2009, ApJS, 183, 295

Teplitz, H. I., Collins, N. R., Gardner, J. P., et al. 2003, ApJS, 146, 209

Treister, E., Virani, S., Gawiser, E., et al. 2009, ApJ, 693, 1713

van der Wel, A., Franx, M., van Dokkum, P. G., et al. 2005, ApJ, 631, 145

van Dokkum, P. G., Franx, M., Förster Schreiber, N. M., et al. 2004, ApJ, 611,703

Vanzella, E., Cristiani, S., Dickinson, M., et al. 2008, A\&A, 478, 83

Vázquez, G. A., \& Leitherer, C. 2005, ApJ, 621, 695

Vieira, J. D., Marrone, D. P., Chapman, S. C., et al. 2013, Natur, 495, 344 
Wang, S. X., Brandt, W. N., Luo, B., et al. 2013, ApJ, 778, 179

Wardlow, J. L., Smail, I., Coppin, K. E. K., et al. 2011, MNRAS, 415, 1479

Weiß, A., De Breuck, C., Marrone, D. P., et al. 2013, ApJ, 767, 88

Weiß, A., Kovács, A., Coppin, K., et al. 2009, ApJ, 707, 1201

Whitaker, K. E., van Dokkum, P. G., Brammer, G., et al. 2013, ApJL, 770, L39
Yang, X., Mo, H. J., van den Bosch, F. C., \& Jing, Y. P. 2005, MNRAS, 356,1293

Younger, J. D., Fazio, G. G., Huang, J.-S., et al. 2009, ApJ, 704, 803

Yun, M. S., Scott, K. S., Guo, Y., et al. 2012, MNRAS, 420, 957

Zheng, W., Mikles, V. J., Mainieri, V., et al. 2004, ApJS, 155, 73 